\title{
Assessing the Progress of Trapped-Ion Processors Towards Fault-Tolerant Quantum Computation
}

A. Bermudez, ${ }^{1,2}$ X. Xu, ${ }^{3}$ R. Nigmatullin, ${ }^{4,3}$ J. O'Gorman, ${ }^{3}$ V. Negnevitsky, ${ }^{5}$ P. Schindler, ${ }^{6}$ T. Monz, ${ }^{6}$ U. G. Poschinger, ${ }^{7}$ C. Hempel, ${ }^{8}$ J. Home, ${ }^{5}$ F. Schmidt-Kaler, ${ }^{7}$ M. Biercuk, ${ }^{8}$ R. Blatt, ${ }^{6,9}$ S. Benjamin, ${ }^{3}$ and M. Müller ${ }^{1}$

${ }^{1}$ Department of Physics, College of Science, Swansea University, Singleton Park, Swansea SA2 8PP, United Kingdom

${ }^{2}$ Instituto de Física Fundamental, IFF-CSIC, Madrid E-28006, Spain

${ }^{3}$ Department of Materials, University of Oxford, Oxford OX1 3PH, United Kingdom

${ }^{4}$ Complex Systems Research Group, Faculty of Engineering and IT,

The University of Sydney, Sydney, Australia

${ }^{5}$ Institute for Quantum Electronics, ETH Zürich, Otto-Stern-Weg 1, 8093 Zürich, Switzerland

${ }^{6}$ Institute for Experimental Physics, University of Innsbruck, 6020 Innsbruck, Austria

${ }^{7}$ Institut für Physik, Universität Mainz, Staudingerweg 7, 55128 Mainz, Germany

${ }^{8}$ ARC Centre for Engineered Quantum Systems, School of Physics,

The University of Sydney, Sydney, NSW 2006, Australia

${ }^{9}$ Institute for Quantum Optics and Quantum Information of the Austrian Academy of Sciences, A-6020 Innsbruck, Austria

(Received 24 May 2017; revised manuscript received 11 August 2017; published 13 December 2017)

A quantitative assessment of the progress of small prototype quantum processors towards fault-tolerant quantum computation is a problem of current interest in experimental and theoretical quantum information science. We introduce a necessary and fair criterion for quantum error correction (QEC), which must be achieved in the development of these quantum processors before their sizes are sufficiently big to consider the well-known QEC threshold. We apply this criterion to benchmark the ongoing effort in implementing QEC with topological color codes using trapped-ion quantum processors and, more importantly, to guide the future hardware developments that will be required in order to demonstrate beneficial QEC with small topological quantum codes. In doing so, we present a thorough description of a realistic trapped-ion toolbox for QEC and a physically motivated error model that goes beyond standard simplifications in the QEC literature. We focus on laser-based quantum gates realized in two-species trapped-ion crystals in highoptical aperture segmented traps. Our large-scale numerical analysis shows that, with the foreseen technological improvements described here, this platform is a very promising candidate for fault-tolerant quantum computation.

DOI: 10.1103/PhysRevX.7.041061

Subject Areas: Quantum Information

\section{INTRODUCTION}

Solving hard computational problems by exploiting the quantum-mechanical laws of nature is one of the goals of current scientific and technological research [1]. To turn this idea into experimental reality, intense research efforts are currently devoted to scale existing small prototypes, which have served for proof-of-principle demonstrations [2], into larger quantum devices capable of processing information quantum mechanically even in the presence of noise and processing errors (i.e., fault tolerantly). This poses a significant challenge from both fundamental and technological perspectives.

Published by the American Physical Society under the terms of the Creative Commons Attribution 4.0 International license. Further distribution of this work must maintain attribution to the author(s) and the published article's title, journal citation, and DOI.
Fundamentally, the quantum-mechanical features responsible for the advantage of these processors with respect to their classical counterparts also give rise to a different behavior with respect to noise and errors, which excludes the straightforward application of classical errorcorrection schemes. Despite these difficulties, the theory of quantum error correction (QEC) [3-5] has shown a welldefined route for the development of large-scale quantum computers. The main ingredients of QEC to combat the detrimental impact of noise are as follows: (i) encoding quantum information redundantly in ever-larger quantum registers and (ii) detecting and correcting errors during a computation without altering the encoded quantum information. Exploiting these ingredients using particular QEC codes, it has been shown theoretically that it is possible to perform quantum-computing sequences of arbitrary complexity fault tolerantly if the noise or error of elementary operations is maintained below a certain threshold [6]. The redundant encoding of the information in these QEC 
protocols, which is required to improve the level of protection against noise for a fault-tolerant computation, can be achieved by either (a) concatenating elementary codes in several layers [5] or (b) storing the information in topological features of registers of increasing size $[7,8]$. The quest is therefore to implement these QEC ideas in quantum devices of increasing sizes.

The first experiments on QEC have implemented the three-qubit [9] and five-qubit [10] quantum codes in nuclear magnetic resonance. Starting from initial experiments on the three-qubit code with trapped ions [11] and superconducting circuits [12], these two platforms have recently been employed to show repetitive error correction [13], faulttolerant error detection with a four-qubit code [14], and small-scale versions of the topological color [15] and surface $[16,17]$ codes. We note, however, that QEC is also being pursued in other platforms [18]. The theory of QEC, described in the paragraph above, defines a clear road map towards the demonstration of fault tolerance in large quantum processors. However, despite this remarkable progress, the hardware platforms are still far away from the sizes that are required to render the errors on the encoded data negligibly small. Hence, it would be desirable to define a set of intermediate QEC goals, which are necessary for the progress towards the fully fledged fault-tolerant quantum computer and can serve as a guiding principle in the experimental design by benchmarking the progress in building and scaling these smaller quantum codes.

A necessary condition for QEC is that the effect of a complete round of error detection and correction must be beneficial for the encoded qubit. This is a nontrivial condition since the effect of an attempt at error correction, while aiming to correct the existing errors, inevitably introduces the risk of new ones. Accordingly, quantifying such a crossover into beneficial or useful QEC, and certifying that it is met in a particular QEC code, will translate into specific requirements on the fidelities of the various gates, measurements, and other internal processes that conform the QEC cycle. This can establish a set of goals that must be achieved by future hardware development. Once this is achieved, another necessary criterion is to verify if the encoding, followed by a complete round of error detection and correction, is beneficial in comparison to the degradation of an unprotected physical qubit subjected to the same sources of physical noise during the time required by the $\mathrm{QEC}$ cycle.

We note that the theoretical studies of the performance of different QEC strategies, quantified by the particular value of the threshold, depend crucially on the assumptions about the underlying platform capabilities and noise models. Using over-simplified noise models, or unrealistic platform capabilities, can lead to an overestimation or underestimation of the correcting power of a given QEC code. Therefore, if we are aiming at assessing and guiding the progress of a particular experimental platform by the above intermediate QEC goals, a very careful microscopic modeling of the noise and the operational errors is required. The objective of this work is to present a detailed study along these lines of trapped-ion quantum processors with current and anticipated future capabilities in the near term.

\section{A. Summary of the results of this work}

In this manuscript, we quantify the above intermediate goals for beneficial QEC by introducing a quantuminformation protocol with a clear and intuitive operational meaning in Sec. II. This protocol can serve as a benchmark scenario to assess the progress of experimental QEC codes.

We focus on trapped-ion implementations of small QEC codes $[11,13-15,19]$ and use the above measure to theoretically assess the methodological and technological improvements that would be required to reach the breakeven point for a logical qubit, i.e., to enter the regime of beneficial QEC. In order to reach this goal, it is of the utmost importance to choose and adapt QEC schemes according to the particular technological advantages and disadvantages of the hardware platform at hand. One must exploit the particular technological strengths and simultaneously mitigate the dominant sources of noise. This requires a detailed knowledge of the state of the art and foreseeable technological improvements, which we discuss in Sec. III. We present a thorough description of an experimental toolbox for QEC using a high-optical-access segmented ion trap to manipulate dual-species ion crystals in a cryogenic environment (see Fig. 1). We consider a universal set of single-qubit and multiqubit entangling gates [20] that differs from the more standard CNOT-based approaches [1]. The current and anticipated performance of these elementary operations, as discussed in detail below, is summarized in Table II. These tools will be combined with spectroscopic decoupling of a subset of ions (i.e., hidingbased approach) and with crystal-reconfiguration techniques (i.e., shuttling-based approach) as summarized in Table IV, which includes splitting ion crystals, shuttling ions across trap segments, and merging two sets of ions into a larger crystal. Together with the possibility of using a dual-species crystal for sympathetic recooling of the ions and stabilizer readout, this toolbox contains all the ingredients required for trapped-ion QEC. In addition to this knowledge of experimental capabilities, assessing the prospects of QEC also requires a detailed modeling of the main sources of noise and errors for these operations, which we address in Sec. IV.

Equipped with this toolbox, we develop different approaches for trapped-ion QEC in Sec. V. We start by describing how multiqubit Mølmer-Sørensen (MS) gates $[22,23]$ can be exploited for efficient stabilizer readout [24], as experimentally demonstrated in Ref. [15]. In the context of fault-tolerant QEC, however, different schemes based on one-qubit gates and two-qubit MS gates would be required. Since fault-tolerant QEC schemes have typically been 


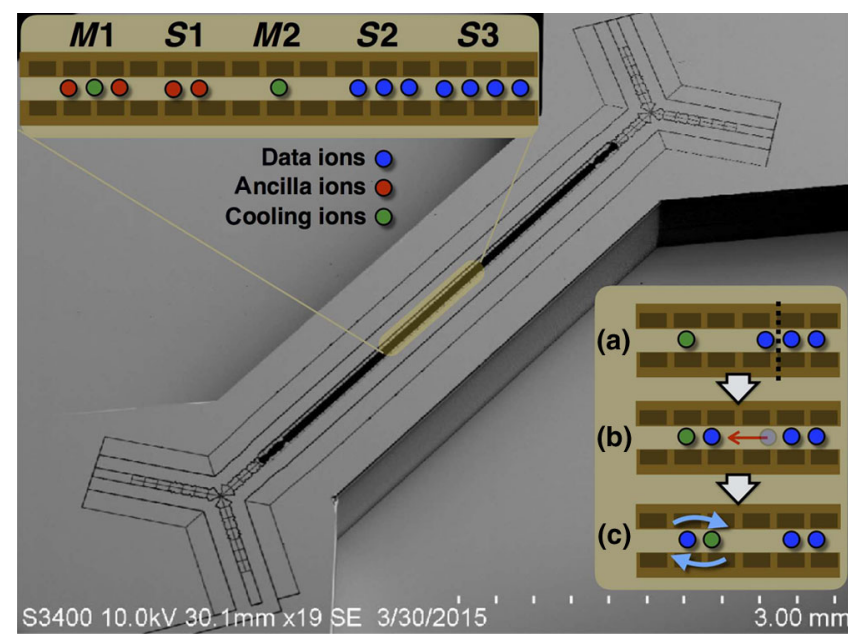

FIG. 1. The Sandia HOA2 trap as a QEC platform: In our envisioned scheme, ${ }^{40} \mathrm{Ca}^{+}$ions (blue and red dots) are cotrapped with ${ }^{88} \mathrm{Sr}^{+}$ions (green dots) in a quantum zone divided into three storage regions $S_{1}, S_{2}, S_{3}$ and two manipulations zones $M_{1}, M_{2}$. Some of the ${ }^{40} \mathrm{Ca}^{+}$ions can be used as data qubits to encode quantum information according to a QEC code (blue dots), while others (red dots) can be used as ancilla qubits for syndrome extraction. The ${ }^{88} \mathrm{Sr}^{+}$ions (green dots) are used as sympathetic coolants to reduce the number of phonons prior to the entangling gates. Possible crystal-reconfiguration operations are shown in the panel in the lower-right corner: (a) splitting of an ion crystal, (b) shuttling of an ion and subsequent merging with another ion to form a crystal, and (c) rotation (swapping) of a mixed species crystal. Schematics of the trap are adapted from a micrograph in Ref. [21].

conceived using single-qubit and two-qubit CNOT gates $[25,26]$, it would be desirable to devise trapped-ion circuits that exploit MS gates directly and to study how errors propagate on those circuits to demonstrate fault tolerance. We address these points by presenting a detailed description of a generic MS-based toolbox for QEC. We apply this toolbox to the seven-qubit topological color code with trapped ions, either using a non-fault-tolerant stabilizer readout with seven data qubits and one additional ancillary qubit (i.e., $7+1$-qubit scheme) based on multiqubit or sequential two-qubit MS gates, or by using fault-tolerant stabilizer readout via MS-based schemes that realize the equivalent of the CNOT DiVincenzo-Shor (DVS) protocol for $7+5$ qubits [25] or the DiVincenzo-Aliferis (DVA) protocol for $7+4$ qubits [26]. Although we have focused on this particular code, we remark that this trapped-ion QEC toolbox for stabilizer readout can be generalized to any other stabilizer QEC code of interest and scaled to larger-size codes in a modular fashion.

The MS-based stabilizer readout is used, in combination with some of the elementary operations of Tables II and IV, as a building block for the development of trapped-ion QEC protocols in Sec. VI. As already outlined above, we explore different scenarios according to varying experimental capabilities:
(1) Shuttling-based protocol.- Here, we consider trapped-ion crystals with either a single or two species of ions species, i.e., data and ancillary qubits being encoded in the same or different atomic species. We develop sequences of crystal-reconfiguration operations and stabilizer mappings to perform a full QEC cycle on a single logical qubit. We explore how the ability of crystal recooling by sympathetic cooling via the ancillary ion at intermediate stages affects the performance of the protocol.

(2) Hiding-based protocol-- Here, we consider the protocols realized in a static ion crystal. Qubits are selectively addressed by shelving inactive ions via spectroscopic decoupling and recoupling pulses, and combined with stabilizer mappings to perform a full QEC cycle on a single logical qubit. We consider encoding of data and ancillary qubits in two different species and the possibility to apply recooling after the readout.

These QEC protocols are complemented with the error model introduced in Sec. IV, which improves upon customary circuit-error models that consider a unique quantum channel for all elementary operations in a QEC cycle. This allows us to perform a detailed study that goes beyond standard, albeit not very realistic, assumptions: (i) We consider that the different gates (including the identity), the state preparation, and the measurements do not take the same amount of time. (ii) We use distinct error channels affecting the different stages of the QEC protocols. For instance, idle qubits are subjected to dephasing in a trapped-ion setup, whereas single- and multiqubit gates are subjected to depolarizing noise. More importantly, (iii) the different channels are not all characterized by a unique error probability. Certainly, single- and multiqubit gates do not have the same error in any known experimental platform. We use a microscopic modeling of the ion crystals to derive the particular expressions or values of the corresponding error rates for each operation. Therefore, our treatment not only goes beyond models that do not consider, or simplify, the occurrence of errors on the syndrome readout, but it also goes beyond the standard so-called circuit-level noise model, which typically makes these over-simplifications.

These sections set the stage for a large-scale numerical analysis that investigates the performance of such protocols in Sec. VII. The criterion introduced for beneficial or useful QEC is used to quantify the three essential requirements that will need to be met in forthcoming experiments for trapped-ion QEC: (i) sufficiently small natural physical error rates from fundamental error sources, (ii) detection and dynamical correction of errors at a fast enough rate, and (iii) sufficiently accurate realizations of unavoidably imperfect error-correction routines so that there is still an overall gain of applying (imperfect) QEC procedures.

Finally, in Sec. VIII, we present our conclusions. 


\section{ASSESSING THE PROGRESS ON QEC BY SMALL QUANTUM CODES}

While small quantum codes have already been demonstrated on different platforms [9-17], it would be of interest to ultimately demonstrate fault tolerance on existing or near-future hardware. However, this would require showing the supremacy (i.e., reducing the error rate) of the small codes with respect to the best-possible unencoded qubits on all representative quantum circuits belonging to a large set of protocols [27]. Depending on the platform, this comparison can be very stringent. For instance, ion-trap processors can use decoherence-free qubits [28] or the $\mu$-metal shielded Zeeman qubits [29] with very long coherence times, such that the error on the identity quantum circuit would be very hard to beat using any small QEC code. Additionally, single-qubit and two-qubit gates with bare qubits have, so far, the smallest achieved infidelities in any experimental platform [30,31], and it also seems unlikely that small QEC codes, with their large overhead in complexity, will be capable of beating them. We thus believe that alternative criteria have to be established, which serve as reasonable guiding principles in the development of future technologies that improve upon existing QEC codes.

\section{A. Breakeven point for useful QEC}

In this section, we introduce the criteria used in our work to judge whether a particular combination of hardware and quantum code can successfully perform QEC, thus sustaining a logical encoded qubit. As mentioned in the Introduction, a first necessary condition that must be verified by any implementation of a QEC code is that the effect of a complete round of error detection and correction proves to be beneficial. In order to make it quantitative, we must define a measure for the quality, or integrity, of a logical qubit.

The fidelity of the logical encoded state subjected to noise or errors $\tilde{\rho}_{L}$ with respect to its ideal form $\rho_{L}=\left|\psi_{L}\right\rangle\left\langle\psi_{L}\right|$, namely, $\mathcal{F}=\left\langle\psi_{L}\left|\tilde{\rho}_{L}\right| \psi_{L}\right\rangle$, and, in particular, how it changes if we perform a QEC cycle on the imperfect $\tilde{\rho}_{L}$ or not, might first appear as a natural measure. However, one encounters difficulties. Consider a logical qubit $\tilde{\rho}_{L}$ that has completely decohered under the effect of independent depolarizing noise on the constituent $n$ physical qubits. The collective entity no longer contains any information about the initial logical state $\left|\Psi_{L}\right\rangle$. Its fidelity is $\mathcal{F}=2^{-n}$ since the system is in an equal mixture of all possible states regardless of the initial encoded state. The problem is that a round of error correction will seem to improve the quality of this logical qubit: It will map all the states in the mixture to either the logical zero $\left|0_{L}\right\rangle$ or the logical one $|1\rangle_{L}$, creating a mixture of these two, $\rho_{\mathrm{QEC}}=\frac{1}{2}\left(|0\rangle_{L}\left\langle\left. 0\right|_{L}+\mid 1\right\rangle_{L}\left\langle\left. 1\right|_{L}\right)\right.$. Consequently, the fidelity will rise to $\mathcal{F}_{\mathrm{QEC}}=\frac{1}{2}$ under an ideal QEC cycle (or close to this number for imperfect correcting circuits). Thus, if we were to select fidelity as our measure for the quality of a logical qubit, we would be faced with the unsatisfactory feature that a logical qubit that has been completely lost, and is free of any meaningful information, seems to be partially recovered.

One might attempt to correct this issue by projecting the $n$-qubit state into the logical subspace and only then computing the fidelity. Nonetheless, this leads to another unsatisfactory feature: One would find an equality in apparent performance between a device that maintains the logical qubit entirely in that subspace and one that allows a large component of the state to leave the subspace, regardless of the nature of the part of the state outside the proper subspace. As we will later remark, the approach we take in this work can be thought of as essentially a more sophisticated variant of this idea.

The alternative measure we employ has a very clear and intuitive operational meaning. It is best illustrated with a quantum-information protocol that separates the role of encoding the logical qubit from the task of reading it. We use the labels Alice and Bob for two entities that have these roles (see Fig. 2). Now suppose that a random qubit state $|\psi\rangle=\alpha|0\rangle+\beta|1\rangle$ is selected, and Alice is instructed to prepare a logical qubit $|\psi\rangle_{L}=\alpha|0\rangle_{L}+\beta|1\rangle_{L}$ using the code of her choice. This logical qubit of $n$ physical qubits $\rho_{L}$ is then subjected to some noise channel, which may have any form, including correlated noise (e.g., spatial or temporal correlations arising from global fluctuating magnetic fields with a characteristic correlation time) and coherent noise (e.g., resulting from over-rotations in single-qubit gate operations). The now-imperfect logical qubit $\tilde{\rho}_{L}$ is presented to Bob, along with the following classical information: "The original state was either $|\psi\rangle=$ $\alpha|0\rangle+\beta|1\rangle$ or $\left|\psi_{\perp}\right\rangle=\beta^{*}|0\rangle-\alpha^{*}|1\rangle$." In other words, Bob is given a choice of two states, the true qubit state and the state that is orthogonal to it. Now, Bob is challenged to make his best guess as to which state the $n$ physical qubits encode: It is a problem of state discrimination with the imperfect logical qubit as the resource. He may use any

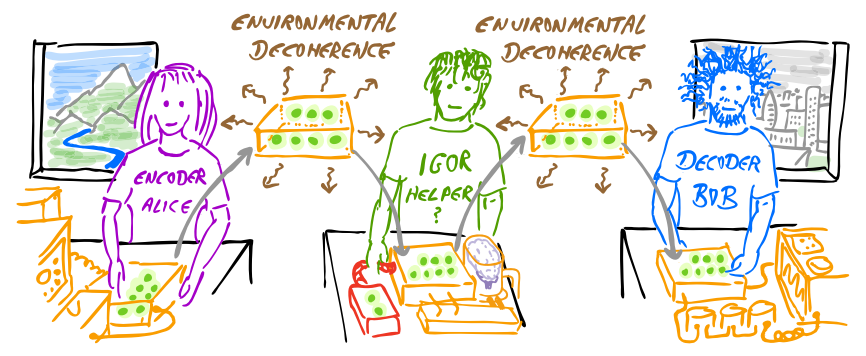

FIG. 2. Cartoon illustration of the protocol for assessing the efficacy of our QEC cycle. Strictly speaking, Alice and Bob have ideal experimental equipment capable of encoding and decoding a quantum state perfectly, whereas only Igor has the imperfections of our real laboratory setting. The stages of the protocol are detailed in Table I. 
physically allowed process in his analysis, and in particular, he can perform error correction, decode to a single physical qubit, and destructively measure it.

For simplicity, we assume that Alice and Bob are ideal agents in the following, i.e., perfect encoding and analysis of the logical qubit. Then, the probability $\mathcal{P}_{\mathrm{B}}\left(\rho_{L}, \tilde{\rho}_{L}\right)$ that Bob guesses correctly will vary only with the quality of the received qubit: An error-free logical qubit will score $\mathcal{P}_{\mathrm{B}, \max }=1.0$, since Bob will certainly succeed in his state-discrimination task, whereas (for example) a logical qubit that has undergone complete depolarization will score $\mathcal{P}_{\mathrm{B}, \min }=0.5$ since Bob can only guess randomly. We define the integrity $\mathcal{I}$ of our memory as simply a scaled probability,

$$
\mathcal{I}=2 \mathcal{P}_{\mathrm{B}}\left(\rho_{L}, \tilde{\rho}_{L}\right)-1 .
$$

The scaling thus provides us with the natural limits of unity for a perfect memory and zero for a memory that provides Bob with no useful information whatsoever.

For any given decoherence model, one can find the probability that Bob will guess correctly given a fully decohered logical qubit. If, as in the following analysis, the decoherence is restricted to a specific channel, then Bob's performance can be higher; i.e., there will be instances in the random selection of the qubit state to be encoded by Alice that happen to be robust against the specific channel. In the cases we are concerned with in the following section, restricting to a purely dephasing environment is an excellent approximation, in which case, if we happen to select $|0\rangle$ or $|1\rangle$ for Alice to encode, the effect of decoherence on the encoded qubit will not degrade Bob's capacity to differentiate: He only needs to measure all qubits in the $z$ basis and determine whether the observed pattern belongs to the set of states associated with $|0\rangle_{L}$ or $|1\rangle_{L}$. Conversely, if we had selected $|+\rangle$ or $|-\rangle$ for Alice to encode, then after full dephasing, Bob would not be able to gain any value from his analysis and his probability of guessing correctly would be 0.5 . His performance when Alice randomly selects qubit states, sampled uniformly over the Bloch sphere, is found to be $\mathcal{P}_{\min }=0.75$ after full dephasing. Therefore, when we plot the average $\mathcal{P}$ for any degree of pure dephasing, we find that it varies in the range $0.75 \leq \mathcal{P}_{\mathrm{B}}\left(\rho_{L}, \tilde{\rho}_{L}\right) \leq 1$.

Armed with this notion of the integrity of a qubit as, essentially, the extent to which its state can be read out by Bob, we now ask the question of whether the QEC cycle is beneficial or harmful by allowing for an imperfect round of error detection and correction prior to Bob's guess $\tilde{\rho}_{L} \rightarrow \rho_{\mathrm{QEC}}$. The full protocol for our measure, where the code to be used (e.g., surface code, 2D color code, etc.) is to be specified, is described in Table I. According to our criterion, the round of imperfect error correction is now deemed to be beneficial if Bob's probability of subsequently discriminating the state correctly is higher when
TABLE I. Protocol for assessing the beneficial role of QEC.

\begin{tabular}{|c|c|}
\hline Step & Action \\
\hline 1 & We select a qubit state at random. \\
\hline 2 & $\begin{array}{l}\text { We require Alice to encode it into the } n \text { physical } \\
\text { qubits of the code. She does so perfectly. }\end{array}$ \\
\hline 3 & $\begin{array}{l}\text { The } n \text { physical qubits are subjected to environmental } \\
\text { noise for a time } \tau / 2 \text {. }\end{array}$ \\
\hline 4 & $\begin{array}{l}\text { Optionally, Igor is asked to apply a full round of } \\
\text { imperfect error correction. }\end{array}$ \\
\hline 5 & $\begin{array}{l}\text { The } n \text { physical qubits are subjected to environmental } \\
\text { noise for a further time } \tau / 2 \text {. }\end{array}$ \\
\hline 6 & $\begin{array}{l}\text { Bob takes the final state of the } n \text { qubits and performs } \\
\text { an analysis so as to make his best guess of the state. } \\
\text { He does so perfectly. }\end{array}$ \\
\hline
\end{tabular}

we indeed perform a round of QEC, vs simply opting not to do so, and allowing the environment to act for time $\tau$ uninterrupted, namely,

$$
\mathcal{P}_{\mathrm{B}}\left(\rho_{L}, \rho_{\mathrm{QEC}}\right)>\mathcal{P}_{\mathrm{B}}\left(\rho_{L}, \tilde{\rho}_{L}\right) .
$$

The breakeven point for a beneficial QEC occurs when Eq. (2) is satisfied. For convenience of exposition, we may imagine that a third party, besides Alice and Bob, is responsible for the cycle of error correction: Since this individual is effectively a flawed assistant for Bob, we use the name Igor after the famous fictional lab assistant (see Fig. 2). Then, our criterion for successful error correction can be summarized as, "Is Igor a help or a hindrance to Bob?"

It is worth noting that Alice's encoding protocol is predetermined and may not vary with the particular choice of qubit she is instructed to encode. Similarly, Igor, who does not have the classical description of the encoded qubit, will always perform the same procedure as he attempts to correct it. Moreover, in all the analysis presented in this paper, we also fix Bob's protocol: He simply performs his own (perfect) round of error correction and then decodes the logical qubit to a single qubit and measures that qubit in the basis of his choice. His optimal basis choice, for all cases considered here, is simply $\left\{|\psi\rangle,\left|\psi_{\perp}\right\rangle\right\}$, and Bob makes his "guess" according to the outcome. Thus, only the final step, the measurement, depends on the choice of encoded qubit that was issued to Alice. It is interesting to observe that with this choice of Bob's protocol, Bob is effectively mapping the state of the $n$ qubits into the logical subspace (with his round of perfect error correction) and then making a guess with a probability of success given by the fidelity of the corrected logical qubit. Thus, our concept of integrity relates directly to the fidelity after the encoded qubit is mapped into the logical subspace via the process of error correction.

Notice that this protocol naturally generalizes to multiple rounds of error correction, i.e., multiple times when the 
imperfect Igor can attempt to help. We simply wait a time $\tau /(m+1)$, have Igor perform his cycle, and repeat until $m$ cycles are performed. After a final wait of $\tau /(m+1)$ so that a total time $\tau$ has elapsed, Bob receives the $n$ physical qubits. For a sufficiently high-performing Igor, and a long enough time $\tau$, it will be beneficial to have multiple rounds. Note that in the numerical simulation of the protocols, as discussed at later stages of this paper, we take into account the finite duration that applications of the QEC cycles require.

Provided that this criterion has been fulfilled, and that QEC is proven beneficial, we can turn to the second desirable property of QEC, namely, that encoding, error detection, and error correction are beneficial in comparison to the degradation of an unprotected physical qubit $\rho=|\psi\rangle\langle\psi| \rightarrow \tilde{\rho}$. For the particular task at hand, this amounts to proving that

$$
\mathcal{P}_{\mathrm{B}}\left(\rho_{L}, \rho_{\mathrm{QEC}}\right)>\mathcal{P}_{\mathrm{B}}(\rho, \tilde{\rho}),
$$

and it would essentially demonstrate that the encoded logical qubit outperforms the quantum memory built with a single unprotected physical qubit of the same sort as those used to form the logical qubit.

\section{TRAPPED-ION EXPERIMENTAL TOOLBOX FOR QEC}

\section{A. Experimental architecture}

The proposed setup consists of a 1D segmented highoptical-access (HOA) ion trap fabricated by Sandia National Laboratories [21] and operated in a cryogenic environment (see Fig. 1). We consider ${ }^{40} \mathrm{Ca}^{+}$ions for hosting the qubits, ${ }^{88} \mathrm{Sr}^{+}$ions for providing the capabilities for sympathetic cooling, and mixed-species readout for syndrome extraction. We consider that ions undergoing the quantum logic operations can be separated and shuttled across the segmented trap array by using high-speed (diabatic), low-excitation protocols in order to minimize cross-talk on neighboring qubits. The required pulsed control of the qubits, system synchronization, measurement, and fast-feedback as required for QEC can be achieved by a custom-engineered high-speed controller.

The choice of the trap is motivated by the requirements for the realization of a QEC code, which demand highfidelity quantum operations on the order of more than 10 ions. Therefore, a microfabricated segmented ion trap that enables multiple trapping zones and versatile ion-crystal reconfigurations is required. This increases the complexity of the trap to a level that can, to date, only be satisfied by quasiplanar trap structures, which reduce the trapping depth such that precautions against ion loss have to be taken. This can be mitigated by lowering the pressure of the vacuum environment by operating the experiment at cryogenic temperatures.
The encoded qubit will be realized in ${ }^{40} \mathrm{Ca}^{+}$ions, which allow for an optical as well as a ground-state qubit. The chosen species enables high-fidelity state detection of the optical qubit because of its simple electronic structure. The optical qubit is formed by the $4 S_{1 / 2}\left(m_{f}=\right.$ $-1 / 2)$ ground state and $3 D_{5 / 2}\left(m_{f}=-1 / 2\right)$ metastable excited state. The excited state has a lifetime of $1.1 \mathrm{~s}$, which sets the upper limit on the qubit storage time [20]. Quantum operations are performed with a laser that is nearly resonant to this transition at a wavelength of about $729 \mathrm{~nm}$. It is also possible to encode a qubit in the two $4 S_{1 / 2}\left(m_{f}= \pm 1 / 2\right)$ Zeeman substates. The coherence of this qubit is only limited by magnetic field fluctuations, where recent improvements resulted in a coherence time of more than one second [29]. State manipulation of this qubit is performed by Raman lasers close to the $4 S_{1 / 2}$ to $4 P_{1 / 2}$ transition. Because of the complexity of the QEC algorithm, a second ion species for sympathetic cooling and stabilizer readout will also be explored. For this, ${ }^{88} \mathrm{Sr}^{+}$ions can be used.

The quality of quantum operations is limited by different processes for the optical and the spin qubit. For the optical qubit, the absolute phase noise of the laser driving the transition limits the achievable coherence, whereas the spin qubit is only sensitive to the differential phase noise in the two Raman laser beams. For the spin qubit, off-resonant excitation of the $4 P_{1 / 2}$ state is a process reducing the gate fidelity, which can only be mitigated by simultaneously increasing the intensity and detuning of the Raman laser beams. However, dynamic control of light at $397 \mathrm{~nm}$ is more challenging than control at a longer wavelength of $729 \mathrm{~nm}$.

We expect that the encoding and QEC of a single logical qubit with a low-distance code can be implemented in a single segmented linear trap with the ion-crystalreconfiguration techniques outlined below. However, multiple logical qubits will likely require a more capable architecture in which ion reconfiguration can be performed more efficiently using three- or four-way junctions. This allows multiple processing regions where syndrome measurements can be performed in parallel, which is also crucial for an extensible QEC architecture.

\section{B. Gate operations}

\section{State of the art}

The experimentally available set of operations considered in this work consists of (i) global laser-driven MS entangling operations $[22,23]$, which can be expressed as

$$
\begin{aligned}
U_{\mathrm{MS}, \phi}(\theta) & =e^{-i(\theta / 4) S_{\phi}^{2}}, \\
S_{\phi} & =\sum_{i=1}^{n}\left(\cos \phi X_{i}+\sin \phi Y_{i}\right),
\end{aligned}
$$

where $\phi$ is controlled by the laser phase and $\theta$ by its intensity and pulse duration. Here, $X_{i}=\sigma_{i}^{x}$ and $Y_{i}=\sigma_{i}^{y}$ are Pauli 
matrices. Additionally, the global laser beams can also drive (ii) global rotations around the Bloch sphere of an individual qubit with a rotation axis in the equatorial plane,

$$
U_{R, \phi}(\theta)=e^{-i(\theta / 2) S_{\phi}},
$$

which are also controlled via the phase, intensity, and pulse duration of the laser beam. Finally, (iii) the addressed acStark shifts result in rotations around the $z$ axis on the Bloch sphere of an individual qubit,

$$
U_{R_{j}, z}(\theta)=e^{-i(\theta / 2) Z_{j}},
$$

where $\theta$ is controlled by the intensity of the off-resonant laser beam, its detuning, and the pulse duration. Here, $Z_{i}=$ $\sigma_{i}^{z}$ is one of the Pauli matrices. This gate set is described in detail in Ref. [20], and a numerical method to find an efficient decomposition of an arbitrary quantum algorithm in a sequence of these gates is presented in Ref. [32]. In the rest of the manuscript, we extensively use the following singlequbit operations:

$$
\begin{aligned}
X_{j}(\theta) & =e^{-(i \theta / 2) X_{j}}, \quad Y_{j}(\theta)=e^{-(i \theta / 2) Y_{j}}, \\
Z_{j}(\theta) & =e^{-(i \theta / 2) Z_{j}},
\end{aligned}
$$

which can be obtained either directly from the available set $Z_{j}(\theta)=U_{R_{j}, z}(\theta)$ or by means of dynamic error-suppression sequences (see Sec. III C). In addition, we use MS gates (4) of $X$ type or $Y$ type acting on a pair of ions, $i$ and $j$. These MS gates are defined as

$$
X_{i, j}^{2}(\theta)=e^{-(i \theta / 2) X_{i} X_{j}}, \quad Y_{i, j}^{2}(\theta)=e^{-(i \theta / 2) Y_{i} Y_{j}},
$$

and obtained (up to a global phase) from $U_{\mathrm{MS}, \phi}(\theta)$ in Eq. (4) by setting $\phi=0$ and $\phi=(\pi / 2)$, respectively; they can be implemented by using spectroscopic decoupling techniques or ion-crystal-reconfiguration steps, such that the MS laser beams only couple to the ion pair $i, j$. Using this notation, $\theta=(\pi / 2)$ MS gates are "fully entangling," as they map the computational basis states of $N$ qubits to GHZ states (up to local unitary rotations) of $N$ qubits. For instance, for two ions, $X_{i, j}^{2}(\pi / 2)=\left(\mathbb{\square}-\mathrm{i} X_{i} X_{j}\right) / \sqrt{2}$, and one finds $X_{i, j}^{2}(\pi / 2)|0\rangle_{i}|0\rangle_{j}=\left(|0\rangle_{i}|0\rangle_{j}-\mathrm{i}|1\rangle_{i}|1\rangle_{j}\right) / \sqrt{2}$. Throughout this manuscript, we use the term multiqubit MS gates to refer to MS gates acting on more than two qubits.

The entangling MS gates are performed on the axial center-of-mass mode of the ion string. This has the advantage that only a single loop in phase space has to be closed to erase unwanted spin-motion entanglement. However, this mode cannot be used for longer ion strings as the ion string approaches a zigzag configuration. This scheme allows for operations generating a maximally entangled GHZ state of up to eight ions, which can be implemented in about $50 \mu$ s for optical qubits with state fidelities of $\{98.6,95.7,81.7\} \%$ for $\{2,4,8\}$ ions [33]. If the limiting factor on the gate quality is phase noise on the laser driving the qubit transition, this could be improved by a laser with smaller phase noise or by switching to groundstate Zeeman qubits, which are only susceptible to the phase difference of the two Raman lasers. Recently, highfidelity entangling operations for two ground-state hyperfine qubits have been demonstrated, reaching infidelities below $10^{-3}[30,31]$.

Carrying out high-fidelity dual-species QIP protocols, such as ${ }^{40} \mathrm{Ca}^{+}$and ${ }^{88} \mathrm{Sr}^{+}$in our case, is generally more difficult than single-species experiments. Complicating factors include a more complex motional mode structure and cooling requirements. However, dual-species entangling gates have already been achieved [34,35], showing Bell-state infidelities of $2 \times 10^{-2}$ and $2 \times 10^{-3}$. Moreover, we have also achieved preliminary dual-species operations for QEC, with ${ }^{40} \mathrm{Ca}^{+}$and ${ }^{9} \mathrm{Be}^{+}$using global optical beams, including experimental approaches for handling dualspecies crystals, as well as initial dual-species gates for $X X$ and $Z Z$ stabilizer readout of a two-ion ${ }^{9} \mathrm{Be}^{+}$crystal with a single ${ }^{40} \mathrm{Ca}^{+}$ion. Preliminary infidelity estimates obtained from Bell state preparation are listed in Table II; SPAM (state preparation and measurement) errors are included in the errors given.

TABLE II. Current and anticipated gate-operation infidelities and durations. Single-qubit operations are a 90-degree rotation on the Bloch sphere; whole entangling operations correspond to fully entangling Mølmer-Sørensen operations (see Sec. III). The reported dual-species operations have been performed in a

\begin{tabular}{|c|c|c|c|c|}
\hline Operation & $\begin{array}{l}\text { Current } \\
\text { duration }\end{array}$ & $\begin{array}{l}\text { Current } \\
\text { infidelity }\end{array}$ & $\begin{array}{c}\text { Anticipated } \\
\text { duration }\end{array}$ & $\begin{array}{c}\text { Anticipated } \\
\text { infidelity }\end{array}$ \\
\hline $\begin{array}{l}\text { Single-qubit } \\
\text { gates }\end{array}$ & $5 \mu \mathrm{s}$ & $5 \times 10^{-5}$ & $1 \mu \mathrm{s}$ & $1 \times 10^{-5}$ \\
\hline $\begin{array}{l}\text { Entangling } \\
\quad(2 \text { qubits })\end{array}$ & $40 \mu \mathrm{s}$ & $1 \times 10^{-2}$ & $15 \mu \mathrm{s}$ & $2 \times 10^{-4}$ \\
\hline $\begin{array}{l}\text { Entangling } \\
\quad \text { (5 qubits) }\end{array}$ & $60 \mu \mathrm{s}$ & $5 \times 10^{-2}$ & $15 \mu \mathrm{s}$ & $1 \times 10^{-3}$ \\
\hline $\begin{array}{l}\text { Dual species } \\
\text { entangling } \\
\text { (2 qubits) }\end{array}$ & $60 \mu \mathrm{s}$ & $3 \times 10^{-2}$ & $15 \mu \mathrm{s}$ & $4 \times 10^{-4}$ \\
\hline $\begin{array}{l}\text { Dual species } \\
\text { entangling } \\
\text { (3 qubits) }\end{array}$ & $80 \mu \mathrm{s}$ & $5 \times 10^{-2}$ & $15 \mu \mathrm{s}$ & $6 \times 10^{-4}$ \\
\hline $\begin{array}{l}\text { Dual species } \\
\text { entangling } \\
\text { (5 qubits) }\end{array}$ & $\ldots$ & $\cdots$ & $15 \mu \mathrm{s}$ & $2 \times 10^{-3}$ \\
\hline Measurement & $400 \mu \mathrm{s}$ & $1 \times 10^{-3}$ & $30 \mu \mathrm{s}$ & $1 \times 10^{-4}$ \\
\hline Recooling & $400 \mu \mathrm{s}$ & $\bar{n}<0.1$ & $100 \mu \mathrm{s}$ & $\bar{n}<0.1$ \\
\hline Qubit reset & $50 \mu \mathrm{s}$ & $5 \times 10^{-3}$ & $10 \mu \mathrm{s}$ & $5 \times 10^{-3} \star$ \\
\hline
\end{tabular}
${ }^{9} \mathrm{Be}^{+}-{ }^{40} \mathrm{Ca}^{+}$crystal. For the parameter marked by the $\star$ symbol, i.e., the anticipated value of the qubit-reset fidelity, numerical simulations were performed for both the value $5 \times 10^{-3}$ and the value $1 \times 10^{-4}$. 


\section{Experimental input for the noise model}

To assess the performance of a QEC procedure with a reasonably sized ion register, one requires a simplified, yet sufficiently realistic, error model. In several studies, circuit noise is assumed to affect the single- and two-qubit gates of the QEC protocol equally. However, in many experimental setups, the leading source of noise affecting idle qubits, single-qubit gates, and entangling operations can be very different, thus requiring more elaborate noise models. The chosen error model in this work includes perfect gate operations followed by a depolarizing channel on the active qubits, and inactive qubits are affected by dephasing noise. We use microscopic calculations and experimental results to set the parameters of this error model, which has the advantage that it can be numerically simulated efficiently even for large qubit registers by using parallelizable Monte Carlo techniques, providing quantitative target gate fidelities. More details on this error model can be found in Sec. IV.

\section{Expected performance}

The required parameters for the chosen noise model can be fixed by the knowledge of (i) the state infidelity for the gates, which describes the strength of the depolarizing noise, and (ii) the duration of the gates, which are used to estimate the effect of dephasing on idling qubits. Table II shows a summary of current and anticipated gate operations for these parameters. The current coherence time on the ground-state qubit is 2 seconds. By improving the magnetic-field stability, it is expected that this can be extended to 10 seconds. The current coherence time on the optical qubit is $200 \mathrm{~ms}$, which is anticipated to be improved to the limit given by the spontaneous decay from the metastable excited state to about 2 seconds.

\section{Dynamic error suppression}

In developing a trapped-ion experimental toolbox for QEC, we are motivated to explore how the capabilities on hand in the laboratory may be crafted to maximize compatibility with the stringent demands on quantum error correction. Our primary objectives are twofold: (1) ensure gate errors are suppressed to rates as low as practicable relative to fault-tolerance thresholds and (2) ensure compatibility of the underlying error model with the mathematical assumptions of fault tolerance in QEC. In our experiments, we therefore routinely turn to open-loop control protocols applied at the physical layer and designed to improve gate performance in advance of QEC.

The strict requirements of fault tolerance on qubit error rates have motivated the development of error-suppressing physical-layer control techniques [36-52] known as dynamic error suppression (DES). In these feedback-free protocols, temporal modulation of the qubit control field is employed in order to effectively average away decoherence induced by environmental fluctuations or control imperfections. These protocols are considered an important complement to QEC [43,53,54], both because of their potential to improve the resource efficiency of QEC and the fact that these protocols work in the presence of noise with strong temporal correlations, a regime that violates most error models underpinning the functionality of QEC. In fact, even in the presence of strong qubit decoherence, DES can extend the effective qubit lifetime by decoupling from slowly varying noise sources. We expect that, in general, targeted application of DES will be implemented at the physical level for qubit manipulation.

\section{Relevant control protocols}

We consider control protocols with diverse historical origins but a common framework for implementation. Physical qubit operations consist of multiple elementary control operations, which are sequentially applied in such a way that the desired target operation (quantum gate) is realized while simultaneously reducing the net sensitivity to error. We treat control protocols by taking the form of an $n$-segment sequence of unitaries, executed over the time period $[0, \tau]$. This implies a partition of the sequence duration $\tau$ into $n$ subintervals $I_{l}=\left[t_{l-1}, t_{l}\right], l \in\{1, \ldots, n\}$, such that the $l$ th control unitary has duration $\tau_{l}=t_{l}-t_{l-1}$. The total operation can thus be expressed as

$$
R(\theta, \phi, \tau):=\prod_{l} U_{\mathrm{R}, \phi_{1}}\left(\theta_{l}\right)=\prod_{l} e^{-i\left(\theta_{l} / 2\right) S_{\phi_{l}}},
$$

where we have used the rotations in Eq. (5) and defined $\theta_{l} \equiv \Omega_{l} \tau_{l}$ in terms of the time-independent Rabi frequency $\Omega_{l}$ during the $l$ th time interval $\left[t_{l-1}, t_{l}\right]$. The resultant generator leads to a rotation of the Bloch vector through an angle $\theta_{l}$ about an arbitrary axis $\mathbf{l}=\left(\cos \left(\phi_{l}\right)\right.$, $\left.\sin \left(\phi_{l}\right), 0\right)$.

The assignment of the relevant control parameters for each segment, $\left\{\Omega_{l}, \tau_{l}, \phi_{l}\right\}$, may be determined by a variety of techniques. "Composite-pulse" constructions address a combination of static pulse-length and off-resonance control errors and are generally implemented via piecewiseconstant phase modulation. Representative sequences include the so-called SK1 and BB1, correcting for pure amplitude errors [55,56], CORPSE for pure detuning errors [56,57], and both reduced CinSK (CORPSE in SK1) and reduced CinBB (CORPSE in BB1) [58] for simultaneous errors. Dynamically corrected gate (DCG) protocols are constructed similarly (via different underlying mathematics, recently unified in Refs. [59-61]) but employ piecewise-constant amplitude and phase modulation of the applied segmented control fields. Representative approaches include the Walsh family of DCGs $[61,62]$.

The approach of producing composite sequential operations achieved through modulation of a control field can be extended to the implementation of two-qubit 
Mølmer-Sørensen gates. Here, one may exploit phase modulation of the driving field used to generate the effective spin-spin coupling via an intermediate bosonic mode [63]. Application of a piecewise-constant, phase-modulation pattern to the driving field permits simultaneous decoupling of "spectator" bosonic modes and the suppression of temporal fluctuations in control amplitude without the need to consider nonlinearities in optical instruments associated with amplitude modulation. The analytic framework in which these gates may be defined rests on a mathematical underpinning similar to that used for the construction of single-qubit DES strategies. This approach is particularly effective in achieving high-order suppression of residual spin-motional entanglement in the ion chain.

DES protocols adapted for idle periods and known as dynamic decoupling [51] are also commonly implemented to correct for a variety of error sources. For instance, we employ spectroscopic decoupling to store idle qubits temporarily in Zeeman sublevels that are not affected by the lasers responsible for QEC gates [15]. In the spirit of refocusing of dynamics, this decoupling is achieved by a sequence composed of $N_{\mathrm{p}}=9$ pulses that can be applied to a set of $\ell$ idle ions to be hidden or unhidden, labeled by $h_{1}, \cdots h_{\ell} \in\{1, \ldots, N\}$. The composite pulse sequence is designed in a way which, to lowest order, echoes out addressing errors due to residual light intensity on neighboring ions [15]-for related recent work on how to further reduce systematically addressing errors see also [64].

\section{Evaluating control performance}

The operational fidelity for an imperfect operation is given by $\mathcal{F}_{\text {av }}(\tau)=\frac{1}{4}\left\langle|\operatorname{Tr}(\tilde{U}(\tau))|^{2}\right\rangle$, following Refs. [65,66], where the error propagator $\tilde{U}(\tau)$ captures the influence of noise and approaches the identity in the limit of vanishing errors. Calculating the fidelity requires the error propagator to be expressed as an infinite series using the so-called Magnus expansion as $\tilde{U}(\tau)=\exp [-\mathrm{i} \Phi(\tau)]$, where the effective error operator $\Phi(\tau)=\sum_{\mu=1}^{\infty} \Phi_{\mu}(\tau)$ at the end of the interaction has expansion terms that take the form of time-ordered integrals over nested commutators of the so-called toggling-frame Hamiltonian. Considering unitary errors, it is convenient to define the error vectors $\boldsymbol{a}_{\mu}(\tau)$ by reexpressing the operators $\Phi_{\mu}(\tau)=\boldsymbol{a}_{\mu}(\tau) \cdot \boldsymbol{\sigma}$ in the basis of Pauli operators [65]. Then, one can expand the exponential in the error propagator to obtain the fidelity in the small noise limit,

$\mathcal{F}_{\text {av }}=1-\left\langle a_{1}^{2}\right\rangle-\left[\left\langle a_{2}^{2}\right\rangle+2\left\langle\boldsymbol{a}_{1} \boldsymbol{a}_{3}^{T}\right\rangle-\frac{\left\langle a_{1}^{4}\right\rangle}{3}\right]+\sum_{k=3}^{\infty} \mathcal{O}\left(\xi^{2 k}\right)$,

with $a_{\mu}^{2}:=\boldsymbol{a}_{\mu}(\tau) \boldsymbol{a}_{\mu}(\tau)^{T}$ the norm square of the error vector. This expression contains a collection of terms with equal magnitude arising from different orders of the Magnus expansion (e.g., $a_{2}^{2}$ vs $a_{1}^{4}$ ). An expression for the leading-order fidelity that keeps terms only to $\boldsymbol{a}_{1}(\tau)$ but approximates the full expansion [62] is given by

$$
\mathcal{F}_{\text {av }}(\tau) \approx \mathcal{F}_{\chi}=\frac{1}{2}\{1+\exp [-\chi(\tau)]\},
$$

where we have defined $\chi(\tau):=\left\langle a_{1}^{2}\right\rangle$. We may conveniently move to the Fourier domain via the formalism of the filtertransfer function using

$$
\chi(\tau)=\frac{1}{2 \pi} \int_{-\infty}^{\infty} \frac{d \omega}{\omega^{2}} \sum_{i=a, d} S_{i}(\omega) F_{i}(\omega) .
$$

Here, we have introduced the noise power spectral densities in the amplitude $(i=a)$ and dephasing $(i=d)$ quadratures, $S_{i}(\omega)$, describing the statistical properties of the environmental noise process afflicting the control operations (see the second column of Table III for different examples).

According to this discussion, the key quantities describing the effect of the control modulation are then $F_{i}(\omega)$; these objects characterize the spectral properties of the applied control and can be calculated analytically for any piecewise-constant sequence $[65,67]$, thus providing a simple quantitative means to compare control protocols of interest in the presence of generic, multiaxis, timedependent noise. Because the net infidelity for an operation is given via an overlap integral of the noise power spectral density $S_{i}(\omega)$, and $F_{i}(\omega)$ for the control, we may describe

TABLE III. Single-qubit DES-protected gates enacting net operation $R(\theta, 0)$, following Ref. [59]. Here, $\phi_{1}=\cos ^{-1}(-\theta / 4 \pi)$, $k=\arcsin [\sin (\theta / 2) / 2], a$ is amplitude noise, $d$ is detuning noise, and $s$ is for simultaneous amplitude and detuning noise. Unless otherwise noted, the Rabi frequency $\Omega$ remains fixed during all segments. The Walsh-modulated DCG sequence (WAMF) [49,61,62] maintains constant segment durations $\tau_{l}$ and employs amplitude modulation of the Rabi rate as described below.

\begin{tabular}{lccccccc}
\hline \hline Composite pulse & Error model & $\left(\theta_{1}, \phi_{1}\right)$ & $\left(\theta_{2}, \phi_{2}\right)$ & $\left(\theta_{3}, \phi_{3}\right)$ & $\left(\theta_{4}, \phi_{4}\right)$ & $\left(\theta_{5}, \phi_{5}\right)$ & $\left(\theta_{6}, \phi_{6}\right)$ \\
\hline SK1 & $a$ & $(\theta, 0)$ & $\left(2 \pi,-\phi_{1}\right)$ & $\left(2 \pi, \phi_{1}\right)$ & $\ldots$ & $\ldots$ & $\ldots$ \\
BB1 & $a$ & $(\theta, 0)$ & $\left(\pi, \phi_{1}\right)$ & $\left(2 \pi, 3 \phi_{1}\right)$ & $\left(\pi, \phi_{1}\right)$ & $\ldots$ & $\ldots$ \\
CORPSE & $d$ & $(2 \pi+\theta / 2-k, 0)$ & $(2 \pi-2 k, \pi)$ & $(\theta / 2-k, 0)$ & $\ldots$ & $\ldots$ & $\ldots$ \\
WAMF & $d$ & $(\Omega, \theta, 0)$ & $(\Omega / 2, \theta / 2,0)$ & $(\Omega / 2, \theta / 2,0)$ & $(\Omega, \theta, 0)$ & $\ldots$ & $\ldots$ \\
Reduced CinSK & $s$ & $(2 \pi+\theta / 2-k, 0)$ & $(2 \pi-2 k, \pi)$ & $(\theta / 2-k, 0)$ & $\left(2 \pi,-\phi_{1}\right)$ & $\left(2 \pi, \phi_{1}\right)$ & $\ldots$ \\
Reduced CinBB & $s$ & $(2 \pi+\theta / 2-k, 0)$ & $(2 \pi-2 k, \pi)$ & $(\theta / 2-k, 0)$ & $\left(\pi, \phi_{1}\right)$ & $\left(2 \pi, 3 \phi_{1}\right)$ & $\left(\pi, \phi_{1}\right)$ \\
\hline \hline
\end{tabular}


these objects using the language of filter design and refer to them as filter-transfer functions.

The filter order characterizes the performance of a filtertransfer function by performing a Taylor expansion of the filter-transfer function about $\omega=0$. Assuming noise with dominant spectral weight at low frequencies, the approximation $F(\omega) \propto(\omega)^{2 p}$ holds for some $p$ associated with the most significant power-law expansion term. The associated control protocol thus defines a high-pass filter with filter order $p-1$. This parameter takes on particular relevance in determining the efficacy of a selected control protocol subject to broadband noise. This filter order must be distinguished from the Magnus order of error cancellation associated with quasistatic errors, which can be understood from the DC limit of our filter-function formalism for constant noise fields. A pulse sequence for which the Magnus expansion terms fulfill $\Phi_{1}^{(\mathrm{DC})}=\ldots=\Phi_{\mu-1}^{(\mathrm{DC})}=0$ is then said to compensate static errors to Magnus order $(\mu-1)$ (see Refs. [61,65]). The residual error is then dominated by terms proportional to the $\mu$ th power in the magnitude of the error scaling. This distinction is particularly important when considering more general expressions for the fidelity beyond leading order, in which contributions to the error from multiple Magnus orders appear in the fidelity and are captured through the exponentiated form of $\mathcal{F}_{\chi}$ in Eq. (11).

\section{Expected performance and protocol selection}

The tools outlined above and detailed in publications including Refs. $[59,62,63,65,66]$ suggest an efficient suppression of gate error rates due to noise processes exhibiting strong temporal correlations. Given realistic error models for dephasing noise and slow control-amplitude drifts, factors of error suppression exceeding about $100 \times$ are projected using state-of-the-art systems and substantiated using both numeric simulations and analytic calculations [59]. Key implementation challenges relate to the calibration of the requisite control phases and amplitudes, generally achieved through if modulation protocols such as IQ or $\Phi M$. The addition of time segments to a basic gate operation or complex modulation patterns introduces new paths for error. The errors that are systematic may be efficiently suppressed by judicious choice of DES strategy. Stochastic errors may accumulate as a result of the more complex protocol, but because of their independence, they scale only approximately linearly with added gate time under DES (a proxy measure for complexity). Therefore, DES has the potential to provide substantial benefits.

Taking into consideration the discussion above, we determine a critical path to selection of appropriate modulation protocols. We first observe that high-order error suppression in the Magnus expansion does not imply highorder time-domain noise filtering and vice versa. This has been validated using experiments on trapped ions [62] and formalized rigorously in Ref. [60]. Given the "whitening" effect of DES protocols on low-frequency-dominated noise, it is naively expected that the residual errors under DES will exhibit lower correlations than would otherwise be achieved. However, the order of error cancellation in the Magnus expansion is the primary determinant of correlations between residual errors that can cause failure of QEC protocols. Accordingly, the choice of a DES strategy will first involve a determination of the requisite Magnus order of error cancellation to suppress residual error correlations, next a determination of the high-frequency behavior of system noise, and finally consideration of how added complexity in high-order DES strategies introduces new pathways for error due to poor pulse calibration. Demonstrations of the suppression of residual error correlations using analyses of randomized benchmarking validate this general approach and will be the subject of a forthcoming manuscript.

\section{Ion-crystal-reconfiguration techniques}

\section{State of the art}

Since the seminal proposal for the Quantum CCD [68], the advent of segmented ion traps and fast multichannel arbitrary waveform generators has enabled the demonstration of ion shuttling operations [69]. These operations need to be performed fast on the time scale set by gate operations. This is required to avoid excessive overhead and decoherence from qubit dephasing, as well as anomalous heating of the ion crystal. On the other hand, motional excitations from shuttling must also be avoided in order not to compromise the phonon-mediated MS entangling operations. Thus, the required waveform generators have to fulfill the requirements of (i) analog update rates below typical trap frequencies, (ii) simultaneous and real-time update of many (10-80) channels, and (iii) superior signal integrity, i.e., low noise at trap frequencies, low glitch impulse areas, and low digital crosstalk. Designs for such devices have been reported in Refs. [70,71]. With segmented traps and waveform generators available, intersegment shuttling of single ions within a few trap periods has been reported for ${ }^{9} \mathrm{Be}^{+}$[72] and for ${ }^{40} \mathrm{Ca}^{+}$[73]. While fast separation has also been reported in Ref. [72], the realization for ${ }^{40} \mathrm{Ca}^{+}$from Ref. [73] has been more challenging because of the low transient minimum trap frequency resulting from the increased mass. Recently, a fast rotation of two ${ }^{40} \mathrm{Ca}^{+}$ions with low resulting excitation, which can be used for reordering the qubit register, has also been demonstrated [74]. These experimental results are summarized in Table IV.

\section{Role of ion-string length on crystal reconfiguration}

The extent to which shuttling operations have to be employed for logical qubit encoding, syndrome readout, error correction, and gate operations depends on the 
TABLE IV. Current and anticipated metrics for different shuttling operations, carried out with ${ }^{40} \mathrm{Ca}^{+}$ions in a multilayer trap (see Sec. III and lower inset of Fig. 1). The axial trap frequency is about $2 \pi \times 1.4 \mathrm{MHz}$, while the radial frequencies are around $2 \pi \times 3 \mathrm{MHz}$. The shuttling is carried out with one ion, while the other operations are carried out with two ions. Note that the 3.6- $\mu$ s duration for low-excitation shuttling is obtained with an amplitude- and phase-calibrated deexcitation kick [75]. Since shuttling duration will not be the bottleneck as compared to other operations, we can anticipate a slightly longer duration of $5 \mu \mathrm{s}$ with similar low excitations, but a smaller calibration effort.

\begin{tabular}{lccc}
\hline \hline Operation & $\begin{array}{c}\text { Shuttling (one } \\
\text { segment) [75] }\end{array}$ & $\begin{array}{c}\text { Separation or } \\
\text { merger [73] }\end{array}$ & $\begin{array}{c}\text { Rotation } \\
{[74]}\end{array}$ \\
\hline $\begin{array}{l}\text { Duration } \\
\text { Excitation axial }\end{array}$ & $3.6 \mu \mathrm{s}$ & $80 \mu \mathrm{s}$ & $42 \mu \mathrm{s}$ \\
$\quad($ phonons) & $<0.1$ & 6 & $<0.3$ \\
$\begin{array}{l}\text { Excitation radial } \\
\quad \text { phonons) }\end{array}$ & $N / A$ & $<0.1$ & $<0.2$ \\
$\begin{array}{l}\text { Anticipated duration } \\
\text { Anticipated excitation } \\
\quad \text { axial (phonons) }\end{array}$ & $5 \mu \mathrm{s}$ & $30 \mu \mathrm{s}$ & $20 \mu \mathrm{s}$ \\
Anticipated excitation & $<0.2$ & $<1$ & $<0.2$ \\
$\quad$ radial (phonons) & $<0.01$ & $<0.1$ & $<0.1$ \\
\hline \hline
\end{tabular}

experimental capabilities to store and coherently manipulate ion crystals of intermediate size. For ion strings of increasing size, addressing errors increase, and the presence of more spectator vibrational modes decreases the fidelities of entangling MS gates [22]. Additionally, for segmented microtraps, the ions are confined in smaller potential wells with increased anharmonicities. Furthermore, the more complex geometry does not always allow for precise micromotion compensation in all spatial directions at a reasonable experimental effort. These two effects can give rise to decreased confinement stability, presumably via parametric resonances. As a consequence, decrystallization and trap loss occur at increased rates, such that this can represent a serious obstacle. To our knowledge, these effects have not been thoroughly investigated or quantitatively characterized.

Therefore, the following particular points have to be addressed by future experimental investigations: (a) the actual extent to which the speed of low-excitation shuttling operations can be increased (see Table IV), (b) the extensibility of low-excitation separation or merging and reordering operations beyond two ions, and to mixed-species scenarios, (c) the scaling of the attainable fidelities of entangling gates with the ion register size, (d) the actual decrease of duration or increase of fidelity of laseraddressed hide or unhide operations, which-as shuttling operations-serve the task of selecting a subset of ions for QEC, and (e) the impact of decay from the metastable state for hidden qubits on the overall error rates, which is to be determined from simulations.

\section{Experimental input for the effective noise model}

In order for simulation results to provide guidance towards the best strategy for logical qubit operation, we need to establish a noise model that captures the essential mechanisms for how shuttling operations contribute to errors but keeps the complexity and computational requirements reasonably small. We thus chose the following model: Each shuttling operation contributes, with a fixed amount of energy, to the radial and axial degrees of freedom of each ion involved in the operation. Despite the fact that the energy is mostly contributed in the form of a coherent oscillator displacement, we assume that there is no fixed phase relation between consecutive displacements corresponding to different shuttling operations. Therefore, the shuttling operations lead to momentum kicks, which heat up the ions. We do not distinguish different collective modes and rather keep track of the mean motional energies of each ion. For merging of ion strings, we assume instantaneous thermalization, such that the total energies are equally distributed among the ions. Whenever entangling gate operations are carried out, we take the motional excitation into account to estimate gate imperfections according to the infidelity estimates discussed in Sec. IV, which consider the excitation on spectator vibrational modes, as well as the excitation of the gate-mediating bus mode.

\section{Expected performance for shuttling operations}

The anticipated improvements are due to ongoing efforts such as the following: (a) Filter undistortion-the distortion induced by second-order low-pass filters on the segment supply lines is partially undone by correcting for the filtertransfer function, at the expense of control voltage amplitude as a resource. This increases the degree of control. (b) Increased control voltage range-the larger segment voltages generated by a second-generation waveform generator will increase the minimum confinement throughout separation or merger operations and enable crystal reordering at larger radial trap frequencies. (c) Ramp generationsoftware for automated voltage ramp generation will find optimized voltage ramps, possibly employing control techniques such as shortcuts to adiabaticity or optimal control [76]. According to these improvements of an existing setup with ${ }^{40} \mathrm{Ca}^{+}$ions, the anticipated key metrics for the different elementary shuttling operations are shown in Table IV.

\section{E. Readout and electronic control}

Protecting a logical qubit via QEC will require repetitive ancilla readout (see Fig. 5) and reset, feedback on the logical qubits, and likely sympathetic recooling of the crystal. Achieving high single-qubit readout fidelity generally requires a trade-off between minimizing the darkand bright-state histogram overlap and minimizing decay 
from the excited to the ground state (as well as repumping of dark states in hyperfine qubits). Additionally, state discrimination must be performed in real time; postprocessing techniques cannot be used to enhance fidelities. A control system, the M-ACTION, has been designed to address these challenges in the context of maintaining a logical qubit: Structured around a fast CPU communicating to field programmable gate arrays (FPGAs), this control system minimizes real-time processing delay, allows rapid prototyping of algorithms in $\mathrm{C}++$, and can feed back to hardware with low latency.

\section{State of the art}

Carrying out experiments on most trapped-ion control systems typically involves describing the experiment on a $\mathrm{PC}$ in a simple domain-specific language, running a simple compiler to produce real-time bytecode, and executing this on a peripheral device such as a FPGA board or PC card $[20,77]$ running a simple finite-state machine. This approach does not support arbitrary feedback requiring nontrivial calculation within latencies comparable to other ion-trapping operations, i.e., well below $10 \mu \mathrm{s}$.

An alternative approach is to design the system to have significant low-level processing power directly at the FPGA board; this allows more complex real-time decisions and calculations without being limited by communication bandwidth and will be essential for QEC and other protocols requiring feedback. This design principle has been implemented in the M-ACTION system [78] used in a number of recent experiments on calcium ions $[79,80]$. The system uses a chip consisting of a FPGA tightly coupled to two physical ARM central processing units, which allows standard $\mathrm{C}++$ to be compiled. Thus, the numerical libraries of $\mathrm{C}++$ can be fully utilized in decision processes, allowing low-latency decisions during experimental sequences [80].

Control electronics, including synthesizers generating qubit drive fields, must be linked to a stable master clock for both synchronization of distributed control electronics and provision of a stable phase reference against which qubit coherence is measured. This is vital because the common decoherence mechanism of dephasing represents a relative measure of the phase coherence of two effective oscillators, as outlined in Ref. [36]. Common approaches to the provision of stable references include the use of an atomic frequency standard with good long-term stability, such as commercial rubidium and cesium clocks, followed by a quartz oscillator providing superior broadband phase noise. Both long-term stability and short-term phase noise represent critical sources of error; analyses have demonstrated that the use of common lab-grade synthesizers serving as system master clocks can produce error rates nearing the percent level in less than $100 \mu$ s. Such error rates are easily suppressed by more than 4 orders of magnitude through appropriate selection of the master clock. In future systems with multiple master clocks, it will be essential to ensure that slowly varying drifts between clocks are minimized to maintain a fixed laboratory reference frame for operations [36].

\section{Building blocks and expected performance}

In the planned QEC scheme, a common step is to map a syndrome onto an ancilla qubit, read out its state, and reinitialize the ancilla as well as cool the ion chain. The dominant source of readout infidelity in both ${ }^{40} \mathrm{Ca}^{+}$and ${ }^{88} \mathrm{Sr}^{+}$optical qubits will likely be background counts for short readout times, which increase the dark and bright histogram overlap. This can be counteracted by increasing the photon collection time or efficiency, such that more photons are collected and the dark and bright histograms become more separated. Spontaneous decay from the D states is another source of infidelity for longer readout times, exceeding $10^{-4}$ after roughly $100 \mu$ s for ${ }^{40} \mathrm{Ca}^{+}$and dominating for very large detection times.

Assuming a reasonable collection efficiency of $0.6 \%$ and a background count rate of $10^{4} / \mathrm{s}$, and considering possible beam intensities similar to those available for ${ }^{40} \mathrm{Ca}^{+}$, the delay incurred in reading out the ${ }^{88} \mathrm{Sr}^{+}$ancilla will be $100-300 \mu$ s with an infidelity of below $10^{-3}$. A readout infidelity of $10^{-4}$ in $150 \mu$ s for the optical transition in ${ }^{40} \mathrm{Ca}^{+}$ has been achieved using Bayesian schemes that incorporate photon arrival times in the state estimate and attempt to identify spontaneous decays [81]. By increasing the photon collection efficiency and thus reducing the detection time to below $20 \mu$ s, this will be attainable with a background count rate of $2 \times 10^{3} / \mathrm{s}$ and collection efficiency of $3.5 \%$.

After readout, the ion chain can be recooled using electromagnetically induced transparency (EIT) cooling on the radial modes [82] and sideband cooling on the bus mode for multiqubit gates. EIT cooling takes several hundred microseconds depending on the geometry, ion-level structure, and initial temperature. The initial temperature depends strongly on the fluorescence lasers: On resonance, they will cause significant heating, whereas by red-detuning and by weakening them, Doppler cooling will occur at the expense of photon counts. Thus, an optimum may be found between readout time, readout fidelity, and heating, such that the total readout and cooling time is minimized.

After EIT cooling, the mean phonon number will already be below $\bar{n}=1$; thus, few sideband-cooling pulses are required. Since cooling times will be at least several hundred microseconds, there is significant time available for classical computation (determining if and where an error has occurred) and feedback latency (preparing the error-correction pulse or pulses) in M-ACTION. If the chain were cooled only once per several readouts, however, these classical delays could become the bottleneck. Computation for a seven-qubit code, even when using Bayesian readout, should take $5 \mu \mathrm{s}$, and feedback latency is around $50 \mu \mathrm{s}$. Ongoing efforts to reduce this will lead to a latency of $1.5 \mu \mathrm{s}$. 
The corrective operation when an error is detected, which involves a single-qubit rotation on a data qubit, is an optional step that can be avoided by altering future gates on that qubit to take the error into account. This requires more classical computation; however, we do not anticipate the computation time being a problem for a single logical qubit. It could, however, result in an increasingly broad tree of sequences. These must either be precomputed and preloaded onto the FPGA hardware or loaded onto the hardware in real time. Preloading the sequences will require more memory on the hardware and is infeasible beyond approximately 5-10 feedback cycles, whereas real-time loading will take up to $1 \mathrm{~ms}$ for tens of pulses. A scalable solution will be real-time loading using either a more efficient encoding scheme or a high-bandwidth communication link.

\section{EFFECTIVE ERROR MODELS FOR ELEMENTARY QEC OPERATIONS IN TRAPPED IONS}

In Sec. VI, we introduce specific trapped-ion protocols to assess the progress of QEC. In this section, we build on our previous discussion of the state-of-the-art and future developments in trapped-ion technology to model the noise on the elementary operations of these QEC protocols by certain quantum channels. As already noted previously in this manuscript, several works on QEC use circuit-error models with a unique quantum channel equally affecting all the elementary operations of the QEC cycle. In this work, we go beyond these assumptions and develop a more involved model with several distinct channels, the parameters of which can be set by microscopic calculations and/or experimental measurements. This model contains certain simplifications or limitations, which we comment on in due course.

\section{A. Dephasing channel for idle qubits}

During the QEC cycles, there are several operations in which the internal states of a subset of qubits are not affected. More specifically, these operations are (i) crystal reconfiguration, leaving all the qubit states unchanged, (ii) single-qubit rotations and Ms entangling gates, which leave the spectator qubits unchanged, and (iii) ancillary qubit measurement and recooling where the data qubits remain idle. In all of these processes, the idling qubits suffer mainly dephasing due to their coupling to the environment, e.g., fluctuating magnetic fields, which can be modeled by the identity followed by a dephasing channel acting on the particular subset of $m$ idle qubits $i_{1}, i_{2}, \ldots, i_{m} \in\{1, \ldots, N\}$. To simplify the model, we assume that the noise channel fulfills the i.i.d. criterion; i.e., it is temporally and spatially uncorrelated. This leads to the usual dephasing channel as described in Ref. [1] but applied to the set of idle qubits $\varepsilon_{\mathrm{d}}(\rho)=\varepsilon_{i_{1}}^{d} \circ \varepsilon_{i_{2}}^{d} \circ \cdots \circ \varepsilon_{i_{m}}^{d}(\rho)$, where

$$
\varepsilon_{i}^{d}(\rho)=\left(1-p_{d}\right) \rho+p_{d} \sigma_{i}^{z} \rho \sigma_{i}^{z}
$$

is a Kraus map and $p_{d}$ is the probability for a single phase flip. It would also be interesting to study spatially correlated dephasing, which does not necessarily imply a faster decoherence as occurs for GHZ states [33], but it also enables almost decoherence-free subspaces in certain codes [15].

One can easily estimate the phase-flip probability by calculating the time evolution of a single qubit subjected to a fluctuating shift of the transition frequency, which is modeled by a stochastic process. Assuming a Markovian regime, one finds $p_{d}=\frac{1}{2}\left(1-e^{-\Gamma_{d} t_{i}}\right) \approx\left(\Gamma_{d} / 2\right) t_{I}$, where $t_{I}$ is the time interval where the qubit remains idle, and $\Gamma_{d}$ is the rate of dephasing. This leads to a dephasing time $T_{2}=1 / \Gamma_{d}$, as measured in Ramsey-interferometry experiments where $\left\langle X_{i}\left(t_{I}\right)\right\rangle=\left\langle X_{i}(0)\right\rangle e^{-t_{I} / T_{2}}$ (see Sec. III B), which yields $p_{d}=t_{I} / 2 T_{2}$.

\section{B. Depolarizing channel for stabilizer mappings}

During the QEC cycles, the stabilizer readout is accomplished by mapping the syndrome information of the data qubits onto ancillary qubits. As described below, this can be accomplished by the combination of two multiqubit MS gates or by a sequence of two-qubit MS gates. We model the leading error of this mapping using a depolarizing channel, as described, for instance, in Ref. [1], after each stabilizer mapping in the QEC protocol. We have explored three types of depolarizing channels affecting $n$ active qubits involved in the MS gates (e.g., $n=5$ active qubits for QEC using multiqubit MS gates, formed by four data and one ancillary qubit labeled by $j_{1}, j_{2}, j_{3}, j_{4}, j_{5} \in\{1, \ldots, N\}$ ):

(i) Independent depolarizing noise.-The first error model consists of independent depolarizing channels $\varepsilon_{\mathrm{MS}}(\rho)=\varepsilon_{j_{1}}^{\mathrm{MS}} \circ \varepsilon_{j_{2}}^{\mathrm{MS}} \circ \cdots \circ \varepsilon_{j_{n}}^{\mathrm{MS}}(\rho)$ acting on each of the active qubits,

$$
\varepsilon_{j}^{\mathrm{MS}}(\rho)=\left(1-p_{\mathrm{MS}}\right) \rho+\frac{p_{\mathrm{MS}}}{3} \sum_{\alpha \in \Lambda_{\alpha}} \sigma_{j}^{\alpha} \rho \sigma_{j}^{\alpha},
$$

where $p_{\mathrm{MS}}$ is the probability for a MS depolarizing error, and $\Lambda_{\alpha}=\{x, y, z\}$. We note that this error model underestimates the occurrence of multiplequbit errors during the entangling gate and can thus overestimate the correcting power of the QEC. Therefore, we have also explored other channels.

(ii) Two-qubit depolarizing noise.-Provided that the $\mathrm{N}$-ion MS gate (4) can be understood as an all-to-all interaction between qubit pairs and is thus localunitary equivalent to applying CNOTs between all $N(N-1) / 2$ ion pairs, an error model that considers single- and two-qubit errors with the same error probability may be more realistic. This will certainly be the case for the QEC schemes based on sequences 
of five-qubit MS gates, where the noise is described by the quantum operation

$$
\begin{aligned}
\varepsilon_{\mathrm{MS}}(\rho)= & \left(1-p_{\mathrm{MS}}\right) \rho+\frac{p_{\mathrm{MS}}}{105} \sum_{i \in \Lambda_{\mathrm{a}}} \sum_{\alpha \in \Lambda_{\alpha}} \sigma_{i}^{\alpha} \rho \sigma_{i}^{\alpha} \\
& +\frac{p_{\mathrm{MS}}}{105} \sum_{j_{1}, j_{2} \in \Lambda_{\mathrm{a}}} \sum_{\alpha, \beta \in \Lambda_{\alpha}} \sigma_{j_{1}}^{\alpha} \sigma_{j_{2}}^{\beta} \rho \sigma_{j_{1}}^{\alpha} \sigma_{j_{2}}^{\beta} .
\end{aligned}
$$

Here, $p_{\mathrm{MS}}$ is the probability for a MS depolarizing error, we have introduced the set of $n$ indices for the active ions $\Lambda_{a}$, and the sum over multiple ion indices excludes coincidences of the pair of indices. For the five-ion MS gate, local unitary equivalent to 10 CNOTs, with each pair of ions potentially undergoing 15 possible Pauli errors (six single-qubit and nine two-qubit Pauli operators), this results in the prefactor $1 / 105$.

(iii) Multiqubit depolarizing noise.-As a worst-case scenario for the schemes based on multiqubit MS gates, we have also explored a model where any five-qubit error can occur with the same error probability because of a faulty five-ion MS gate. This can be described by the quantum operation

$$
\begin{aligned}
\varepsilon_{\mathrm{MS}}(\rho)= & \left(1-p_{\mathrm{MS}}\right) \rho+\frac{p_{\mathrm{MS}}}{1023} \\
& \times \sum_{j \in \Lambda_{\mathrm{a}} \alpha \in \tilde{\Lambda}_{\alpha}} \sigma_{j_{1}}^{\alpha} \sigma_{j_{2}}^{\beta} \sigma_{j_{3}}^{\gamma} \sigma_{j_{4}}^{\kappa} \sigma_{j_{5}}^{\zeta} \rho \sigma_{j_{1}}^{\alpha} \sigma_{j_{2}}^{\beta} \sigma_{j_{3}}^{\gamma} \sigma_{j_{4}}^{\kappa} \sigma_{j_{5}}^{\zeta},
\end{aligned}
$$

where $p_{\mathrm{MS}}$ is the probability for a MS depolarizing error to occur, and we have introduced $\boldsymbol{j}=\left(j_{1}, j_{2}, j_{3}, j_{4}, j_{5}\right), \quad$ and $\quad \boldsymbol{\alpha}=(\alpha, \beta, \gamma, \kappa, \zeta) \quad$ in $\tilde{\Lambda}_{\alpha}=\{0, x, y, z\}$. Here, the sum over multiple ion indices excludes coincidences of any indices; we have introduced $\sigma^{0}=\rrbracket_{2}$ as the identity operation; and the sum over possible Pauli errors excludes the global identity $\alpha=\beta=\gamma=\kappa=\zeta=0$ (i.e., no error), thus giving rise to a total of $4^{5}-1=1023$ possible Pauli error configurations.

To estimate how the error probability of the above depolarizing channels $p_{\mathrm{MS}}$ depends on the different experimental parameters, we calculate the state fidelity of the ideal MS gate followed by each of the depolarizing channels in Eqs. (14) and (15), $\mathcal{F}=\left\langle\Psi_{t}\left|\varepsilon_{\mathrm{MS}}\left(U_{\mathrm{MS}}\left|\Psi_{0}\right\rangle\left\langle\Psi_{0}\right| U_{\mathrm{MS}}^{\dagger}\right)\right| \Psi_{t}\right\rangle$, where the ideal gate produces a GHZ-type state $\left|\Psi_{t}\right\rangle=$ $U_{\mathrm{MS}, \phi}(\pi / 2)\left|\Psi_{0}\right\rangle$.

For the (i) independent depolarizing channel in Eq. (14), one obtains $\mathcal{F}=\left(1-p_{\mathrm{MS}}\right)^{5}+\frac{1}{30}\left(1-p_{\mathrm{MS}}\right)^{3} p_{\mathrm{MS}}^{2}+$ $\frac{1}{15}\left(1-p_{\mathrm{MS}}\right) p_{\mathrm{MS}}^{4} \approx 1-5 p_{\mathrm{MS}}$ for $p_{\mathrm{MS}} \ll 1$. In this case, only the processes where no error occurs contribute to the fidelity at the lowest order in $p_{\mathrm{MS}}$, such that the error probability is simply $p_{\mathrm{MS}}=(1-\mathcal{F}) / 5$. For the (ii) two-qubit depolarizing channel that includes oneand two-qubit errors with the same probability, Eq. (15), one finds $\mathcal{F}=1-\frac{95}{105} p_{\mathrm{MS}}$, such that $p_{\mathrm{MS}}=105(1-\mathcal{F}) / 95$. In this case, processes with no error and two Z-type errors leave the GHZ state invariant and contribute with the same order in $p_{\text {MS }}$. Finally, for the (iii) multiqubit depolarizing channel that includes all five-qubit errors with the same probability, Eq. (16), one finds $\mathcal{F}=1-\frac{1008}{1023} p_{\mathrm{MS}}$, such that $p_{\mathrm{MS}}=1023(1-\mathcal{F}) / 1008$. In this case, processes with no error, and two or four Z-type errors leave the GHZ state invariant and contribute with the same order in $p_{\mathrm{MS}}$.

The probability of the depolarizing channel can then be extracted by comparing it to the GHZ infidelity $\varepsilon=1-\mathcal{F}$ obtained by a microscopic Hamiltonian modeling the evolution of the trapped-ion MS gate [22]. In this way, one can include possible sources of noise and experimental imperfections that lead to evolutions that depart from the ideal MS gate $[22,23]$. We now discuss three different sources of infidelity.

(a) Gate infidelity due to the motional excitations.-The employed MS gate utilizes a bichromatic laser-ion interaction that simultaneously drives the blue and red motional sidebands corresponding to the c.m. axial mode. This acts as a bus mode that mediates an all-to-all qubit-qubit interaction, Eq. (4), capable of generating the aforementioned GHZ states. The motional excitation of this mode, as well as the presence of additional vibrational modes of the ion chain, lead to an infidelity caused by two main sources: (i) off-resonant couplings to the sidebands of spectator modes and (ii) fluctuation of the effective Rabi frequency of the laser-ion coupling due to the motional excitations of bus and spectator modes, i.e., Debye-Waller factors. If one assumes, as argued in Sec. III D, that there is a fast equilibration after ion-reconfiguration operations, resulting in a thermal vibrational state with a mean phonon number that increases after each particular crystal reconfiguration according to Table IV, it is possible to estimate the infidelity of the $N$-ion fully entangling MS gate $\mathcal{F}=1-\varepsilon_{m}$ as

$$
\begin{aligned}
\varepsilon_{m} \approx & \frac{\pi N\left(\delta-\omega_{z}\right)}{2 \omega_{z}^{2} t_{\mathrm{g}}} 0.8(\bar{n}+1) \\
& +\frac{\pi^{2} N(N-1) \eta^{4}}{8 N^{2}}\left(1.2 \bar{n}^{2}+1.4 \bar{n}\right),
\end{aligned}
$$

where $\omega_{z}$ is the c.m. axial mode frequency with mean phonon number $\bar{n}, \delta$ is the symmetric detuning of the bichromatic laser beams with respect to the electronic transition, $t_{\mathrm{g}}$ is the gate time, and $\eta=k_{\mathrm{L}} / \sqrt{2 m \omega_{z}}$ is the single-ion axial Lamb-Dicke parameter. The first term in this equation represents the infidelity due to unclosed phase-space trajectories of the spectator modes, whereas the second one is from the decrease of the Rabi frequency due to the thermal background of all modes, i.e., the DebyeWaller factor. Note that, although all the modes of the ion 
crystal participate in the infidelity, the error can be bounded with quantities that are characterized by the mean phonon number of the c.m. mode.

In order to apply this error estimate, Eq. (17), to the different steps of the QEC cycle based on crystal reconfiguration, we assume that the gate time $t_{g}\left(\bar{n}_{f}\right)$ after a number of crystal reconfigurations that increase the mean phonon number to $\bar{n}_{f}$ is modified with respect to the optimized gate time $t_{g}\left(\bar{n}_{0}\right)$ in Table II, where $\bar{n}_{0} \approx 0$ after laser cooling. This is accomplished by modifying the laser detuning, such that phase-space trajectories of the c.m. mode are still closed for the modified time

$$
t_{g}\left(\bar{n}_{f}\right)=t_{g}(0)\left(1+\frac{\eta^{2}(2 \bar{n}+1)}{N}\right) .
$$

Therefore, the gates become slightly slower the higher the mean phonon number is. Note that this delay will not result in an appreciable change in the dephasing, Eq. (13), that idle qubits suffer for each stabilizer readout, and one can assume that $2 t_{g}(0)$ is the dephasing time for idle qubits during each $N$-ion stabilizer mapping [see Eq. (13) above].

This motional infidelity $\varepsilon_{m}$ can become the leading source of error in the QEC protocol where the ancillary (readout) and physical ions are of the same species and are shuttled, merged, split, or rotated during the QEC cycle to extract the syndrome. Accordingly, recooling of the motion of the ion crystal via the ancillary ion cannot be exploited, as the scattered light would perturb the quantum state encoded in the data qubits. In this situation, the motional excitation of the ion string resulting from the different reconfiguration steps can become very large (see Table IV), yielding a motional infidelity that overcomes other possible sources of noise.

(b) Gate infidelity due to magnetic-field and laserintensity fluctuations.-Another possible source of noise in the MS gate is caused by fluctuations between the qubit frequency and the laser frequency, as well as laser-intensity fluctuations. We model these two sources of noise by stochastic processes that yield fluctuations of the qubit frequencies, and of the laser coupling strengths to the motional sidebands of the MS scheme, respectively. If one assumes that the time correlations of these processes decay much faster than the gate time (i.e., Markovian assumptions), then the gate infidelity can be expressed as $\mathcal{F} \approx 1-\left(\varepsilon_{d}+\varepsilon_{I}\right)$, where $\varepsilon_{\mathrm{I}}$ is the error due to intensity fluctuations and $\varepsilon_{\mathrm{d}}$ is the dephasing error due to, e.g., fluctuating magnetic fields experienced by the qubits during the gate. Such an error can be approximated by

$$
\varepsilon_{d} \approx 2 \Gamma_{d} t_{\mathrm{g}} \sum_{i, j} e^{-\left|z_{i}^{0}-z_{j}^{0}\right| / \xi_{c}}
$$

where $\Gamma_{d}$ is the rate of dephasing leading to a dephasing time $T_{2}=1 / \Gamma_{d}, z_{i}^{0}$ are the positions of the ions in the string, and $\xi_{\mathrm{c}}$ is a typical length scale over which magneticfield fluctuations are correlated; i.e., for $\xi_{c}=0$, we have local noise and $\varepsilon_{d} \approx 2 t_{\mathrm{g}} N / T_{2}$, whereas for $\xi_{c} \gg\left|z_{1}^{0}-z_{N}^{0}\right|$, we have global magnetic-field fluctuations and $\varepsilon_{d} \approx$ $2 t_{g} N^{2} / T_{2}$ would be the dephasing rate affecting a $\mathrm{GHZ}$ state. We note that, for the different mappings of the syndrome information into the ancilla qubits, the actual dephasing error will lie between these two limits, and its particular value will depend on the collective state of the qubits at the instants of time where the MS gates are applied. To simplify the description, we consider a conservative, worst-case scenario and use an error rate $\varepsilon_{d} \approx 2 t_{g} N^{2} / T_{2}$ consistent with the values reported in Table II.

Finally, intensity fluctuations during the gate will have two effects. On the one hand, the time dependence of the fluctuations can lead to a residual spin-motion entanglement due to imperfect closure of the phase-space trajectories of the bus c.m. mode. On the other hand, the acquired phase that depends on the area of the enclosed trajectory may also differ from the one required to generate the desired GHZ state. These two sources of error are accounted for, in corresponding order, by means of the following expression:

$$
\varepsilon_{\mathrm{In}} \approx \Gamma_{\mathrm{In}} t_{g} \eta^{2}\left(\bar{n}+\frac{1}{2}\right)+\Gamma_{\mathrm{In}} t_{g} \frac{\eta^{2}(N-1)}{4} .
$$

Here, $\Gamma_{\text {In }}$ is the rate of intensity fluctuations, obtained through its zero-frequency power spectral density, and it sets a typical time scale for the effects of intensity noise $T_{\text {In }}=1 / \Gamma_{\text {In }}$. We adjust this parameter to be consistent with the fidelities reported in experiments (see Table II).

In the QEC protocols based on two species, we use a different ion species for the ancillary and data qubits. Thus, we can exploit the ancillary ion for intermediate sympathetic recooling of the ion crystal. In any case, the population of vibrational modes remains small prior to the stabilizer mapping via MS gates. Hence, the (i) error due to thermal motional excitation in Eq. (17) will not be leading, but instead, it will contribute, together with other sources of error. We also consider (ii) dephasing and (iii) intensity fluctuations during the gate as additional sources of gate infidelity following Eqs. (19) and (20). Therefore, the MS-gate infidelity that can be used to extract the probability of the depolarizing channels corresponds to $\varepsilon=\varepsilon_{m}+\varepsilon_{d}+\varepsilon_{I}$.

\section{Depolarizing channel for spectroscopic decoupling}

Because of the use of spectroscopic decoupling protocols (see Sec. III C), in the noise model used in this study, we do not consider residual errors on the neighboring ions, and we model the error in this decoupling process by independent single-qubit depolarizing channels acting 
on each of the qubits being hidden or unhidden, $\varepsilon_{h}(\rho)=$ $\varepsilon_{h_{1}} \circ \varepsilon_{h_{2}} \circ \cdots \circ \varepsilon_{h_{m}}(\rho)$, where

$$
\varepsilon_{h}(\rho)=\left(1-p_{h}\right) \rho+\frac{p_{h}}{3} \sum_{\alpha \in \Lambda_{\alpha}} \sigma_{h}^{\alpha} \rho \sigma_{h}^{\alpha},
$$

and $p_{h}$ is the error probability. Taking the current and expected single-qubit gate infidelities $\varepsilon_{1}$ reported in Table II into account, we can estimate the probability of the depolarizing channel for spectroscopic decoupling as $p_{h}=9 \varepsilon_{1}$.

\section{Bit-flip channel for measurement and reset}

The QEC cycles also require a measurement and reset of the ancillary qubit. Faulty measurement or reset can be modeled by a bit-flip channel, as introduced in Ref. [1], that acts on the set of $n_{a}$ ancillary qubits $\left\{a_{1}, a_{2}, \cdots a_{n_{a}}\right\} \in$ $\{1, \ldots, N\}$, namely, $\varepsilon_{b}(\rho)=\varepsilon_{a_{1}} \circ \varepsilon_{a_{2}} \circ \cdots \circ \varepsilon_{a_{n_{a}}}(\rho)$, where

$$
\varepsilon_{a}(\rho)=\left(1-p_{\mathrm{b}}\right) \rho+p_{\mathrm{b}} \sigma_{a}^{x} \rho \sigma_{a}^{x},
$$

with $p_{b}$ the probability for a bit-flip error to occur. We estimate the value of this probability through the infidelities for measurement $p_{b}=\varepsilon_{\text {meas }}$ and qubit reset $p_{b}=\varepsilon_{\text {res }}$ reported in Table II.

\section{TRAPPED-ION TOPOLOGICAL QEC AND FAULT TOLERANCE}

\section{A. Basic properties of the seven-qubit color code}

We focus on the development of trapped-ion QEC protocols to implement a logical qubit based on the seven-qubit color code, assessing their potential to be useful for QEC by the operational measure described in Sec. II. The seven-qubit code constitutes an enabling building block of two main routes towards fault-tolerant quantum computation (FTQC). On the one hand, it is equivalent (up to local unitaries) to the seven-qubit Steane code $[1,5]$ and can, as such, be used as an elementary unit to achieve more and more robust logical qubits by means of concatenation. On the other hand, it constitutes the smallest, though functionally complete, representative of the class of 2D topological color codes [8], for which logical qubits of increasing robustness can be achieved by using codes defined in lattices of increasing size. We note that topological codes typically display higher error thresholds in comparison to concatenated ones, thus offering a practical and very promising route towards large-scale QEC.

One of the goals of our study is the identification of the accuracy requirements of the experimental building blocks used to realize complete QEC cycles on the logical qubit. For instance, a series of limitations on the experimental approach used in Ref. [15], such as a large overhead in spectroscopic decoupling operations, has already been identified. By a numerical analysis of the operational measures in Eqs. (2) and (3), we aim at deriving quantitative estimates on the experimental requirements to make a QEC based on this protocol beneficial. Moreover, the measure will also allow us to benchmark the performance of other protocols that avoid spectroscopic decoupling. In this sense, exploring a variety of protocols for this code is an ideal test bed for the development of key tools, which would be equally required in the implementation of other small- and medium-size quantum codes, such as the ninequbit Bacon-Shor code (see, e.g., Ref. [83]) or the rotated nine-qubit surface code of distance 3 [84].

Let us briefly summarize a few central properties of the seven-qubit color code that are relevant for the QEC protocols developed and studied below. This code allows one to store and manipulate a $k=1$ logical qubit, which is redundantly encoded in entangled states distributed over $n=7$ physical qubits. The code has a logical distance of $d=3$ and thus allows one to detect and correct at least $t=1$ arbitrary error (phase and/or bit-flip error) on any of the seven physical qubits. The code belongs to the family of CSS codes [4,5] and thus allows one to independently detect and correct bit- and phase-flip errors. Errors are identified by measuring the corresponding error syndrome, which is deduced from the sets of three four-qubit $Z$ - and $X$-type stabilizer operators associated with the three plaquettes of the code (see Fig. 3). If we denote the set of Pauli matrices of the physical qubits as $\left\{X_{i}=\sigma_{i}^{x}\right.$, $\left.Y_{i}=\sigma_{i}^{y}, Z_{i}=\sigma_{i}^{z}\right\}_{i=1}^{n}$, the stabilizers are

$$
\begin{aligned}
& S_{x}^{(1)}=X_{1} X_{2} X_{3} X_{4}, \quad S_{x}^{(2)}=X_{2} X_{3} X_{5} X_{6}, \quad S_{x}^{(3)}=X_{3} X_{4} X_{6} X_{7}, \\
& S_{z}^{(1)}=Z_{1} Z_{2} Z_{3} Z_{4}, \quad S_{z}^{(2)}=Z_{2} Z_{3} Z_{5} Z_{6}, \quad S_{z}^{(3)}=Z_{3} Z_{4} Z_{6} Z_{7} .
\end{aligned}
$$

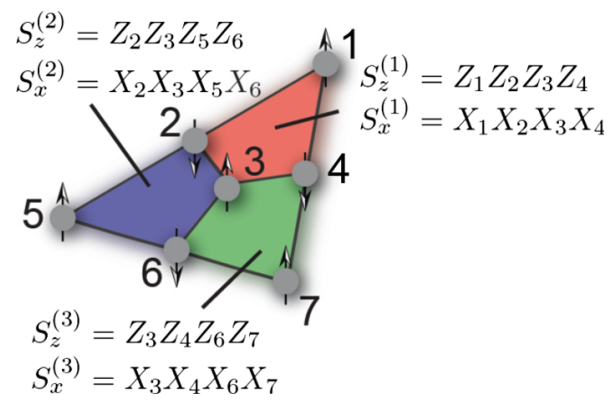

FIG. 3. Scheme of the seven-qubit color code: One logical qubit is embedded in seven data qubits forming a $2 \mathrm{D}$ triangular colorcode structure of three plaquettes. The code space is defined via $S_{i}^{x}$ and $S_{i}^{z}$ stabilizer operators (generators), each acting on a plaquette that involves four physical qubits. Logical operators such as $Z_{L}=\prod_{i} Z_{i}$, and similarly the other logical single-qubit Cliffordgate generators $X_{L}:=\prod_{i} X_{i}, H_{L}:=\prod_{i} H_{i}=\prod_{i}(1 / \sqrt{2})\left(X_{i}+Z_{i}\right)$, and $S_{L}:=\prod_{i} S_{i}^{\dagger}=\prod_{i} e^{-i(\pi / 4)\left(1-Z_{i}\right)}$, can be realized in a transversal, i.e., bit-wise, way. 
Logical states are encoded in the code space, which is defined as the simultaneous eigenspace of eigenvalue +1 of the set of all $s=6$ stabilizer generators (23), such that $k=$ $n-s=1$ coincides with the number of encoded qubits. Logical qubits employing larger distance codes, and thus allowing for the correction of multiple errors, can be constructed by encoding a logical qubit in larger lattice structures involving more physical qubits [8]. An interesting representative of this procedure is the distance- 5 color code (of 4-8-8 type [8]), which encodes a single logical qubit in 17 physical qubits arranged on a 2D lattice structure of eight plaquettes.

A distinguishing feature, as compared, e.g., to Kitaev's surface code $[85,86]$, is that the color code [8] permits a transversal realization of the entire group of Cliffordgate operations [1]. Thus, the realization of a logical Clifford gate on the logical qubit amounts to a bit-wise application of the corresponding gates on all physical qubits $X_{L}=\prod_{i} X_{i}, Z_{L}=\prod_{i} Z_{i}$ and, similarly, the other logical single-qubit Clifford-gate operations, such as the Hadamard $H_{L}$ and $K$ gate $K_{L}$. This property not only facilitates the practical implementation of logical gate operations, but, more crucially, it also prevents an uncontrolled propagation of errors through the quantum hardware - a central requirement to ultimately reach the FTQC regime. A universal set of logical gate operations can be achieved by complementing the Clifford operations with a single non-Clifford gate. For 2D color codes such an additional gate operation, e.g., the $\mathrm{T}$ gate [1] by magicstate injection [87], involves a quantum-state teleportation process between the register of system qubits and an ancilla qubit.

\section{B. Trapped-ion alternatives to CNOT-based QEC}

In this section, we develop trapped-ion alternatives to CNOT-based schemes for the readout of the four-qubit stabilizer operators of the color code, Eq. (23). This is the essential operation in a QEC cycle, which consists of measuring all $X$ - and $Z$-type stabilizers and performing conditional operations on the physical qubits. These readout schemes are also essential to encode a particular qubit state: Starting from $\otimes_{i}|0\rangle$, one would measure all of the $X$-type stabilizers, perform conditional operations to project onto the code subspace, and apply a single-qubit rotation at the logical level followed by the required QEC cycles.

In this section, we start by describing rules for the propagation of errors in circuits involving MS gates. These rules are used to understand the properties of schemes that work with a single ancillary ion (i.e., non-fault-tolerant schemes) and use either five-ion [Eq. (4)] or two-ion [Eq. (8)] MS entangling gates. The motivation to study these schemes is to gain insight on how important it is to avoid the direct occurrence of multiqubit errors in QEC protocols [15]. By using five or four ancillary qubits and sequences of two-ion MS gates, it is also possible to implement a trapped-ion version of the CNOT-based schemes for fault-tolerant stabilizer readout by DiVincenzo-Shor (DVS) [25] and DiVincenzo-Aliferis (DVA) [26], respectively. The main goal of exploring these schemes is to assess under which conditions, and in which experimental parameter regimes, such fault-tolerant protocols might offer advantages in reaching the breakeven point for useful QEC. Let us remark that the techniques presented here can easily be generalized to any other stabilizer of a different QEC code. Therefore, they will be an essential ingredient of future trapped-ion efforts for QEC.

\section{MS-gate error propagation}

For the construction of fault-tolerant quantum circuits, it is important to understand how errors propagate from one qubit to others by means of the entangling gate operations. The circuit identities in the left panel of Fig. 4 show the wellknown propagation of $X$ - and $Z$-type single-qubit errors through CNOT gates. Analogous error-propagation properties can be derived for fully entangling two-ion $X_{i, j}^{2}(\pi / 2)$ and $Y_{i, j}^{2}(\pi / 2)$ MS gates [Eq. (8)] (see right panel of Fig. 4). Errors of the same type as the basis of the MS gate commute with the gate operation, e.g., $X_{i, j}^{2}(\pi / 2) X_{i}=X_{i} X_{i, j}^{2}(\pi / 2)$, and thus do not propagate to the second qubit. On the contrary, errors of a type different from the MS-gate basis, e.g., a $Z_{i}$ occurring before a Ms gate $X_{i, j}^{2}(\pi / 2)$, are converted into an error of the type that is complementary to the error type, i.e., into a $Y_{i}$ error in this case. In addition, this triggers the creation of an error on the second qubit involved in the gate, i.e., here an $X_{j}$ error. This can be seen

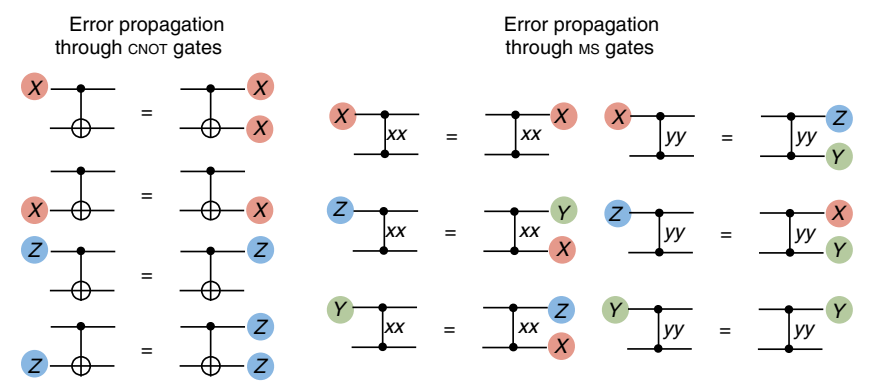

FIG. 4. Error propagation through CNOT and MS gates. (Left panel) An incoming Pauli error of the $X(Z)$ type at the control (target) qubit propagates onto a target (control) error of the same $X(Z)$ type after a CNOT gate. Conversely, an incoming Pauli error of the $Z(X)$ type at the control (target) qubit does not propagate into the target (control) qubit. (Right panel) Error propagation through an MS gate up to phase factors irrelevant for fault-tolerant arguments. The left column corresponds to $X X$-type MS gates $X_{i, j}^{2}$, whereas the right column describes $Y Y$-type MS gates $Y_{i, j}^{2}$. Pauli errors that anticommute with the MS-gate basis are rotated and propagated to the other qubit. Pauli errors in the same MSgate basis do not propagate. 


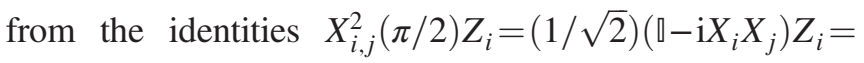

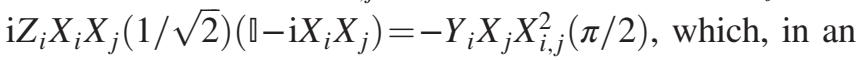
analogous fashion, also hold for $Y$-type MS gates and the other types of errors. Note that, in contrast to the CNOT-gate operation, the MS gate is symmetric in the indices of the two qubits; i.e., these propagation rules hold equally for errors arriving on the second qubit of the gate, e.g., $Y_{i, j}^{2}(\pi / 2) Z_{j}=Y_{i} X_{j} Y_{i, j}^{2}(\pi / 2)$.

\section{Non-fault-tolerant stabilizer readout}

In this section, we start by reviewing the scheme for stabilizer readout of the seven-qubit code [15], which uses a single-ancilla ion and multiqubit MS gates. We then extend this scheme to a protocol that is based on two-ion MS gates, which will be used to explore how important it is to avoid the direct occurrence of multiqubit errors from the multiion MS gates.

(i) Multiqubit MS stabilizer readout.-The readout of the stabilizers [Eq. (23)] can be accomplished by mapping the syndrome information of the data qubits onto a single ancillary qubit using two global five-ion MS gates [Eq. (4)] and an intermediate single-qubit rotation via a local ac-Stark shift [Eq. (6)] since

$$
\begin{aligned}
& U_{\mathrm{MS}, 0}(-\pi / 2) U_{\mathrm{R}_{j}, z}(-\pi / 2) U_{\mathrm{MS}, 0}(\pi / 2) \\
& =e^{i(\pi / 4) Z_{j} \Pi_{i \neq j} X_{i}},
\end{aligned}
$$

as shown in Ref. [24]. By applying this sequence to the ancillary ion and a particular subset of four qubits $\left\{j_{1}, j_{2}, j_{3}, j_{4}\right\}$ belonging to a particular plaquette stabilizer [Eq. (23)], one can map all stabilizers $\left\{S_{\alpha}^{(1)}, S_{\alpha}^{(2)}, S_{\alpha}^{(3)}\right\}_{\alpha=x, z}$ onto the ancillary qubit. For instance, for the first $X$-type stabilizer, one finds $U_{\mathrm{MS}, 0}(-\pi / 2) U_{\mathrm{R}_{0}, z}(-\pi / 2) U_{\mathrm{MS}, 0}(\pi / 2)=$ $\exp \left(\mathrm{i}(\pi / 4) Z_{0} S_{x}^{(1)}\right)$. The stabilizer information can then be measured by performing a Ramsey-type sequence on the ancillary qubit (see Fig. 5). The ancilla qubit is initialized in $|0\rangle_{0}$, and after the pulse $U_{R_{0}, \phi}=U_{R, \phi}(\theta / 2) U_{R_{0}, z}(\pi) U_{R, \phi}(-\theta / 2) U_{R_{0}, z}(-\pi)$, one maps the stabilizer information into the ancilla using the above scheme. Finally, after applying the pulse on the ancilla qubit $U_{R_{0}, \phi+\pi / 2}=$ $U_{R, \phi+\pi / 2}(\theta / 2) U_{R_{0}, z}(\pi) U_{R, \phi+\pi / 2}(-\theta / 2) U_{R_{0}, z}(-\pi)$, one measures it in the computational basis $M_{0}^{z}$, obtaining \pm 1 when the ancilla qubit is found in state $|0\rangle_{0}$ or $|1\rangle_{0}$, respectively. These outcomes correspond to the \pm 1 eigenvalue information of the corresponding stabilizer.

(ii) Two-qubit MS stabilizer readout.-Let us now introduce a circuit for the readout of a four-qubit stabilizer based on two-ion MS gates. Such a circuit could be constructed by compiling the known

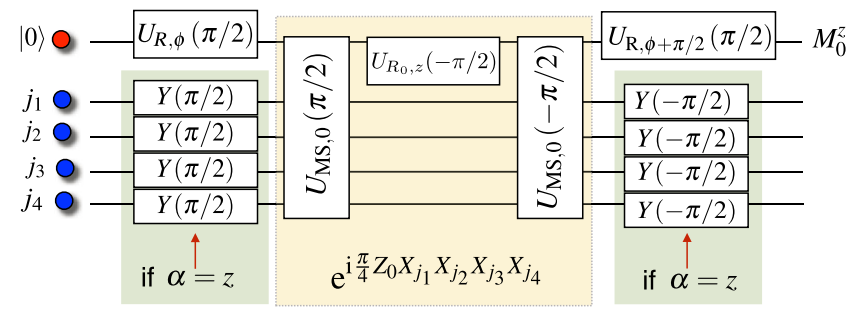

FIG. 5. Stabilizer readout based on multiqubit MS gates: The yellow box contains two five-ion MS gates, interspersed by a single-qubit rotation of the ancillary qubit around the $Z$ axis. This sequence realizes a coherent mapping of the $+1 /-1$ eigenvalue information of $X$-type stabilizers [Eq. (23)] onto two orthogonal states of the ancilla qubit (red dot), initially prepared in a superposition state on the equator of the Bloch sphere. Note that the basis of the initial rotation $U_{R, \phi}(\pi / 2)$ required to prepare the ancilla qubit in this way, specified by the angle $\phi$, can be arbitrary as long as the final rotation $U_{R, \phi+\pi / 2}(\pi / 2)$ of the ancilla is shifted by $\pi / 2$. In other words, the relative phase between these two single-qubit pulses on the ancilla needs to be well defined, but there does not need to be a fixed phase reference between the addressed laser, driving resonant single-qubit rotations, and the lasers driving global rotations and the entangling MS gate. For the readout of $Z$-type stabilizers [Eq. (23)], the data qubits must be rotated via $Y( \pm \pi / 2)$ at the beginning and at the end of the mapping, to effectively switch between $X$ - and $Z$-type stabilizers.

circuits based on four CNOTs, using the fact that a two-ion MS gate is equivalent to a two-qubit CNOT, up to local unitary operations [88]. Alternatively, one can construct such circuits that minimize the building blocks by noticing that

$$
\begin{aligned}
U_{x}^{(i, j)} & :=\left|x_{+}\right\rangle\left\langle\left. x_{+}\right|_{i} \otimes \mathbb{q}_{j}+\mid x_{-}\right\rangle\left\langle\left. x_{-}\right|_{i} \otimes \mathrm{i} X_{j}\right. \\
& =X_{j}\left(\frac{-\pi}{2}\right) X_{i, j}^{2}\left(\frac{\pi}{2}\right), \\
U_{y}^{(i, j)} & :=\left|y_{+}\right\rangle\left\langle\left. y_{+}\right|_{i} \otimes \mathbb{q}_{j}+\mid y_{-}\right\rangle\left\langle\left. y_{-}\right|_{i} \otimes \mathrm{i} Y_{j}\right. \\
& =Y_{j}\left(\frac{-\pi}{2}\right) Y_{i, j}^{2}\left(\frac{\pi}{2}\right),
\end{aligned}
$$

where $\quad\left|x_{ \pm}\right\rangle=(|0\rangle \pm|1\rangle) / \sqrt{2}$ and $\quad\left|y_{ \pm}\right\rangle=$ $(|0\rangle \pm \mathrm{i}|1\rangle) / \sqrt{2}$. This identity shows that a combination of a two-qubit MS gate between an ancillarydata qubit pair $(0, j)$ and a single-qubit $\pi / 2$ pulse on the data qubit $j$ acts essentially as a rotated version of a CNOT gate. Thus, by sequentially applying this pair of operations between the ancilla qubit and the four plaquette qubits, we realize the mapping of certain stabilizers $S_{x}^{(m)}=X_{j_{1}} X_{j_{2}} X_{j_{3}} X_{j_{4}}$ onto the ancilla qubit. Considering that the ancilla qubit is initially in $|0\rangle_{0}$, while the data qubits are in an arbitrary state $|\psi\rangle$, we find 


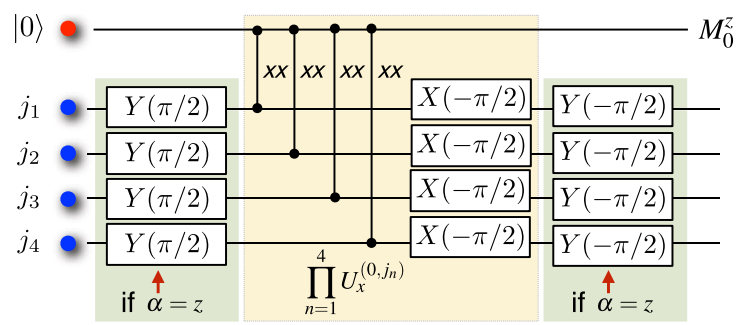

FIG. 6. Stabilizer readout based on sequential two-qubit MS gates: $X$-type $(\alpha=x)$ and $Z$-type $(\alpha=z)$ stabilizer readout circuits, using sequential two-ion MS $X_{0, j}^{2}$ gates [Eq. (8)], depicted by solid lines with black dots between the single ancilla qubit and each of the data qubits involved in a particular stabilizer, and with an $X X$ label that defines the basis of the entangling gate [Eq. (4)]. The MS gates have to be combined with single-qubit rotations $X_{j}(\pi / 2), Y_{j}( \pm \pi / 2)$ on the data qubits [Eq, (25)] to achieve the stabilizer mapping [Eq. (26)]. The measurement of the ancillary qubit in the computational basis is denoted as $M_{0}^{z}$.

$$
\begin{aligned}
\prod_{n=1}^{4} U_{x}^{\left(0, j_{n}\right)}|0\rangle_{0}|\psi\rangle= & |0\rangle_{0} \frac{1}{2}\left(1+S_{x}^{(m)}\right)|\psi\rangle \\
& +|1\rangle_{0} \frac{1}{2}\left(1-S_{x}^{(m)}\right)|\psi\rangle
\end{aligned}
$$

Hence, the two possible values of the stabilizer \pm 1 can be directly inferred by measuring the ancilla qubit $M_{0}^{z}$ in the $Z$ basis, such that the initial and final Ramsey pulses of the multiqubit readout of Fig. 5 are not required any longer. Similarly, Z-type stabilizer operators can be measured by interchanging the basis of the data (plaquette) qubits from $X$ to $Z$ before and after applying the above sequence of gates, $Y_{j}(\pi / 2) X_{j} Y_{j}(-\pi / 2)=-Z_{j}$. The resulting circuits for measuring $X$ - and $Z$-type stabilizer operators are shown in Fig. 6.

Note, however, that using a single ancillary qubit does not allow for a fault-tolerant measurement of the stabilizers for the seven-qubit code. The reason for this is that a singlequbit error can propagate to the set of data qubits and lead to two errors therein. In the subsequent round of QEC, these will then lead to a logical error (see Fig. 7 for details). Similar effects would occur for the previous scheme based on five-ion MS gates, also forbidding a fault-tolerant behavior.

\section{Fault-tolerant DiVincenzo-Shor stabilizer readout with two-ion MS gates}

In this section, we develop a fault-tolerant version of the stabilizer readout using two-ion MS gates. Let us consider the CNOT-based approach by DVS [25], in which a four-qubit ancilla GHZ-type state ("cat state") is prepared, verified, and subsequently coupled transversally, and thus fault tolerantly, to the respective four data qubits. This scheme requires, besides the seven data qubits of the color code, five additional ancilla qubits: four for the ancilla GHZ state (indices $a_{1}, a_{2}, a_{3}$, and $\left.a_{4}\right)$ and one extra ancilla qubit $\left(a_{0}\right)$ for verifying the GHZ state in a measurement. The preparation, verification, transversal coupling, and decoding for the readout of a single stabilizer can be accomplished by 12 CNOT gates and a couple of Hadamard gates. Since the CNOT for two ion qubits in a larger register can be constructed using a single MS gate and four single-qubit rotations $Y_{a_{n}}(-\pi / 2) X_{a_{n}}(-\pi / 2) X_{j_{n}}(-\pi / 2) X_{a_{n}, j_{n}}^{2}(\pi / 2) Y_{a_{n}}(\pi / 2)=$ $e^{i \pi / 4}\left(|0\rangle\left\langle\left. 0\right|_{a_{n}} \otimes \mathbb{1}_{j_{n}}+\mid 1\right\rangle\left\langle\left. 1\right|_{a_{n}} \otimes X_{j_{n}}\right)\right.$, the straightforward translation of the DVS protocol onto a trapped-ion hardware would require 12 MS gates and 50 single-qubit rotations. Let us now discuss, step by step, an alternative Ms-based approach that reduces the total number of gates (see Figs. 8 and 9).

(i) Preparation of the ancilla GHZ state.-The fourqubit GHZ state can be prepared by a sequence of two-ion $X X$ and $Y Y$ MS gates acting on the ancilla qubits initially prepared in $\left|\psi_{0}\right\rangle=\left|0_{a_{1}}, 0_{a_{2}}, 0_{a_{3}}, 0_{a_{4}}\right\rangle$. A single $X X$ MS gate leads to a Bell state of the ancilla qubits $a_{1}, a_{2}$, rewritten as $X_{a_{1}, a_{2}}^{2}(\pi / 2)\left|\psi_{0}\right\rangle=$ $(1 / \sqrt{2})\left(\left|x_{+}\right\rangle_{a_{1}}\left|y_{-}\right\rangle_{a_{2}}+\left|x_{-}\right\rangle_{a_{1}}\left|y_{+}\right\rangle_{a_{2}}\right)\left|0_{a_{3}}, 0_{a_{4}}\right\rangle=:\left|\psi_{1}\right\rangle$. A subsequent $Y Y$ entangling gate applied to $a_{2}$ and $a_{3}$ extends this state into a three-qubit GHZ-type state, namely, $Y_{a_{2}, a_{3}}^{2}(\pi / 2)\left|\psi_{1}\right\rangle=(1 / \sqrt{2})\left(\left|x_{+}\right\rangle_{a_{1}}\right.$ $\left.\left|y_{-}\right\rangle_{a_{2}}\left|x_{-}\right\rangle_{a_{3}}+\left|x_{-}\right\rangle_{a_{1}}\left|y_{+}\right\rangle_{a_{2}}\left|x_{+}\right\rangle_{a_{3}}\right\rangle\left|0_{a_{4}}\right\rangle=:\left|\psi_{2}\right\rangle$. Finally, a $X$-type MS gate on $a_{3}$ and $a_{4}$ produces a GHZ-type state $X_{a_{3}, a_{4}}^{2}(\pi / 2)\left|\psi_{2}\right\rangle=:\left|\psi_{3}\right\rangle$, where we have introduced (a)

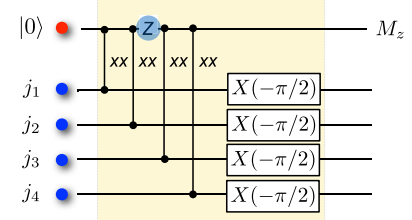

(c)

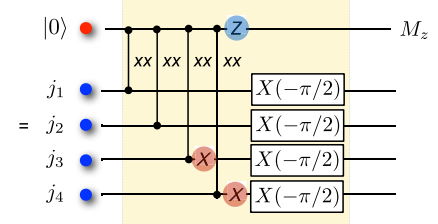

(b)

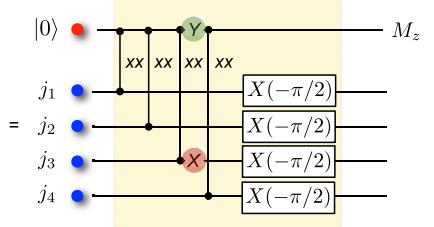

(d)

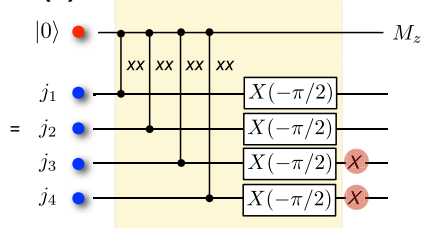

FIG. 7. Non-fault tolerance of single-ancilla MS-based stabilizer readout: Illustration of an error event, in which a single phase-flip error $Z$ on the ancilla qubit occurs between the second and the third MS gates (a). This error propagates into the data qubit layer (b) after the third MS gate, where it results in two bit single-qubit flip errors on the data qubits (c). These two errors correspond ultimately to a logical bit-flip error (d) that cannot be corrected by the code. 


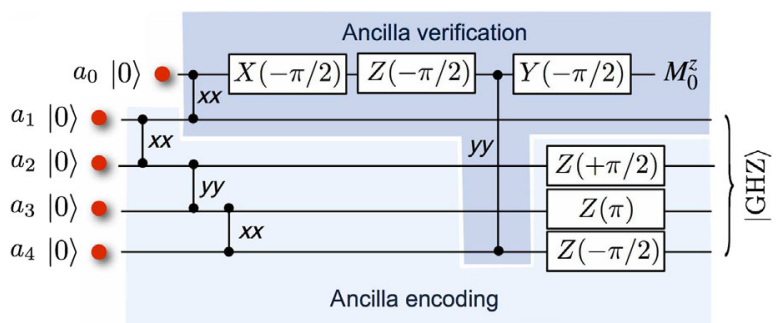

FIG. 8. MS-based circuit for preparation and verification of a GHZ state in the DiVincenzo-Shor scheme: The first three MS gates create a four-qubit GHZ state, which is, up to singlequbit $Z$ rotations, equivalent to the desired GHZ state $(1 / \sqrt{2})\left(\left|x_{+}, x_{+}, x_{+}, x_{+}\right\rangle+\left|x_{-}, x_{-}, x_{-}, x_{-}\right\rangle\right)$. The state is verified by coupling it via two additional MS gates involving the first and the fourth ancilla qubit to a verification qubit. Note that the $Z$-type rotation on the ancilla verification qubit is incorporated to remove a relative phase in the GHZ state. Some parts of the circuit can be executed in parallel, such as part of the verification circuit, while the GHZ state is still being built up.

$$
\begin{aligned}
\left|\psi_{3}\right\rangle= & \frac{1}{\sqrt{2}}\left(\left|x_{+}\right\rangle_{a_{1}}\left|y_{-}\right\rangle_{a_{2}}\left|x_{-}\right\rangle_{a_{3}}\left|y_{+}\right\rangle_{a_{4}}\right. \\
& \left.+\left|x_{-}\right\rangle_{a_{1}}\left|y_{+}\right\rangle_{a_{2}}\left|x_{+}\right\rangle_{a_{3}}\left|y_{-}\right\rangle_{a_{4}}\right),
\end{aligned}
$$

which can be converted into a standard GHZ-type state after its verification.

(ii) Verification of the GHZ state.-The GHZ-type state $\left|\psi_{3}\right\rangle$ [Eq. (27)] can be verified by coupling the first $a_{1}$ and fourth $a_{4}$ ancilla qubits to the verification ancilla qubit $a_{0}$ via $X$ - and $Y$-type MS gates, which, together with local unitary rotations on the verification qubit, yield the operations $U_{x}^{\left(a_{1}, a_{0}\right)}$ and $U_{y}^{\left(a_{4}, a_{0}\right)}$ in Eq. (25), respectively. The expressions (25) show

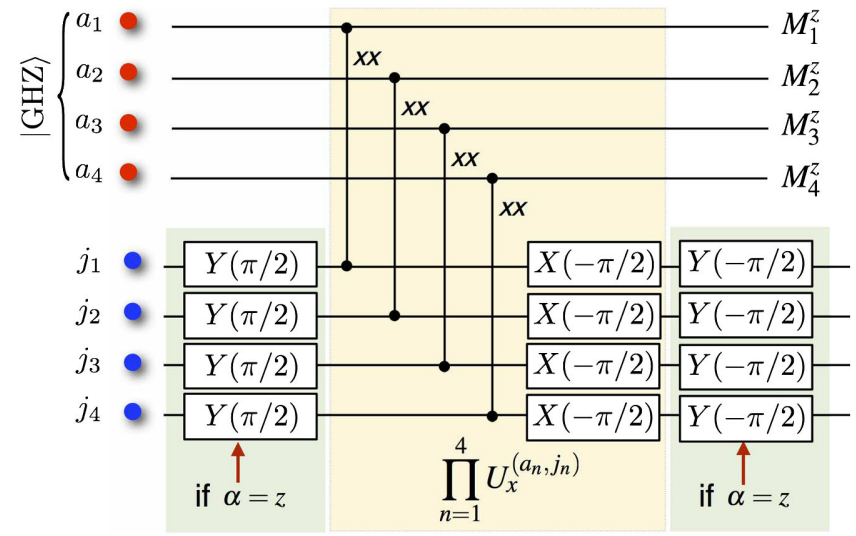

FIG. 9. MS-based circuit for transversal coupling in the DiVincenzo-Shor scheme: The stabilizer $S_{\alpha}^{(n)}$ information is encoded in the relative phase of the ancilla GHZ state $(1 / \sqrt{2})\left(\left|x_{+} x_{+} x_{+} x_{+}\right\rangle \pm\left|x_{-} x_{-} x_{-} x_{-}\right\rangle\right)$by a sequence of two-ion MS gates and local rotations (25). This relative phase is revealed by the even or odd parity of the Z-basis measurements of the four ancilla qubits. that, under these two operations, the ancilla verification qubit initially prepared in $|0\rangle_{a_{0}}$ is either not flipped at all (i.e., first component of the GHZ state $|\psi\rangle_{3}$ ) or flipped twice (i.e., second component of the GHZ state $\left|\psi_{3}\right\rangle$ ), gaining an additional phase shift that can be compensated with a local $Z$ rotation. Therefore, for a perfect preparation of the GHZ-type state $U_{y}^{\left(a_{4}, a_{0}\right)} Z_{a_{0}}(-\pi / 2) U_{x}^{\left(a_{1}, a_{0}\right)}\left|0_{a_{0}}\right\rangle\left|\psi_{3}\right\rangle=\left|0_{a_{0}}\right\rangle\left|\psi_{3}\right\rangle$, and the verification qubit should ideally end up in $|0\rangle_{a_{0}}$, which can be checked by measuring $M_{0}^{z}=+1$ in the computational basis.

The state $\left|\psi_{3}\right\rangle$ is finally converted by local $Z$ rotations into the desired GHZ state $Z_{a_{2}}(\pi / 2) Z_{a_{3}}(\pi) Z_{a_{4}}(-\pi / 2)\left|\psi_{3}\right\rangle=|\mathrm{GHZ}\rangle$, where we have introduced

$|\mathrm{GHZ}\rangle=\frac{1}{\sqrt{2}}\left(\left|x_{+}, x_{+}, x_{+}, x_{+}\right\rangle+\left|x_{-}, x_{-}, x_{-}, x_{-}\right\rangle\right)$,

which will be coupled transversally to the data qubits. The circuit for the MS-based scheme used for the preparation as well as verification of the ancilla GHZ state is shown in Fig. 8.

(iii) Coupling of the ancilla GHZ state to the data qubits.-To realize the readout of an $X$-type stabilizer operator, the verified four-qubit ancilla GHZ state is then coupled transversally to the corresponding four data qubits. Again, using the operators in Eq. (25), it can be shown that the sequence of pairwise unitaries $U_{x}^{(4)}=\prod_{n=1}^{4} U_{x}^{\left(a_{n}, j_{n}\right)} \quad$ leads to $\left|\psi_{4}\right\rangle=$ $U_{x}^{(4)}|\mathrm{GHZ}\rangle|\psi\rangle=(1 / \sqrt{2})\left(\left|x_{+}, x_{+}, x_{+}, x_{+}\right\rangle|\psi\rangle+\mid x_{-}, x_{-}\right.$, $\left.\left.x_{-}, x_{-}\right\rangle S_{x}^{(m)}|\psi\rangle\right)$, where we have introduced an arbitrary basis state of the four data qubits $|\psi\rangle$. Accordingly, these sequential operations map the \pm 1 eigenvalue information of a stabilizer $S_{x}^{(m)}=X_{j_{1}} X_{j_{2}} X_{j_{3}} X_{j_{4}}$, and thus part of the error syndrome, onto the relative phase of the ancillas $\quad\left|\psi_{4}\right\rangle=(1 / \sqrt{2})\left(\left|x_{+}, x_{+}, x_{+}, x_{+}\right\rangle \pm \mid x_{-}\right.$, $\left.\left.x_{-}, x_{-}, x_{-}\right\rangle\right)|\psi\rangle$. This relative phase of +1 or -1 will result in even or odd parity of the combined outcome $\left(M_{1}^{z}, M_{2}^{z}, M_{3}^{z}, M_{4}^{z}\right)$ of a subsequent measurement of the four ancilla qubits in the $Z$ basis as shown in Fig. 9. In this figure, we also show the additional local rotations that must be performed for the measurement of Z-type stabilizers. As customary for stabilizer codes, the same circuit can be used as a building block to prepare the state of the encoded qubit.

To demonstrate the fault-tolerant nature of the constructed circuit, we must show that, if at most a single error (single-qubit error, two-qubit gate error, or measurement error) occurs anywhere in the circuit, it will not result in an uncorrectable error on the data qubits that would yield 
a logical error. Note that $X$ errors resulting at the output of the circuit are not dangerous, as they can only result in a wrong relative sign of the GHZ state, which is later coupled to the data qubits. This can ultimately result in stabilizer measurement errors. Such stabilizer measurement errors are taken care of by applying several rounds (up to three) of readout to reliably decode the error syndrome. With respect to the fault tolerance of the circuit, the key point is that such $X$ errors never propagate to data qubits since they commute with the $X X$ MS gates used during the coupling stage, both for $X$ - and $Z$-type stabilizer readout.

In contrast, undetected phase-flip errors $(Z)$ generated during the preparation and verification of the GHZ state in Fig. 8 will propagate to the data qubit layer during the coupling step in Fig. 9, resulting in bit-flip errors $(X)$. If two bit-flip errors are introduced into the code, this will result in an uncorrectable logical error. However, the preparation and verification circuit is constructed in such a way that any combination of two phase-flip errors is detectable, as it will necessarily result in an $M_{0}^{z}=-1$ measurement of the ancilla verification qubit $a_{0}$. If this is the case, the GHZ state must be discarded, and another attempt is made at preparing and verifying the required ancilla GHZ state. It is tedious but straightforward to show that all dangerous two-qubit phase-flip errors that can affect the data qubits are equivalent to a $Z_{3} Z_{4}$ error and will be detected in the verification step through the outcome $M_{0}^{z}=-1$. Note that a $Z_{1} Z_{2}$ error is equivalent to a $Z_{3} Z_{4}$ error, as the resulting two bit-flip errors in the code are equivalent up to an $S_{x}$ stabilizer. Three-qubit phase-flip errors, e.g., $Z_{2} Z_{3} Z_{4}$, are equivalent to a single phase-flip error $Z_{1}$ using the same argument, which propagates to the data layer but only results in a single $X_{1}$ bit-flip error. This is an allowed process, as singlequbit errors will be picked up and corrected for in the next QEC round to leading order.

\section{Fault-tolerant DiVincenzo-Aliferis stabilizer readout with two-ion MS gates}

In this section, we develop a fault-tolerant MS-based version of the DVA approach [26], which was originally introduced in terms of CNOT gates. Similar to the DVS scheme discussed above, an ancilla GHZ state (28) is initially prepared by a sequence of MS gates. The main difference of the DVA protocol is that its verification is postponed until the end of the readout step. Hence, the GHZ state is coupled transversally to the data qubits, after which the ancilla state is decoded and measured to obtain the stabilizer information. Importantly, the decoding circuit is constructed in such a way that it also allows one to unambiguously detect the occurrence of two single-qubit errors that have propagated to the data qubits, potentially causing a logical error. If such a situation is detected, the corresponding two-qubit error-correction operation is either physically applied to the data qubits or used on a software level to update the Pauli frame.

DiVincenzo and Aliferis argue that, by postponing the verification step involving measurements to the end, this scheme can be highly beneficial and avoid bottlenecks when the measurements of qubits are much slower than gate operations [26], which is typically the case in trappedion hardware (see Tables II and IV). Furthermore, only four ancilla qubits are needed, as compared to the five needed for the DVS scheme of Fig. 8. Another nice feature is that the verification is not of a stochastic nature, which can considerably simplify the time control and synchronization in a larger QEC protocol. Previous studies have aimed at a comparison of DVS and DVA CNOT-based schemes for the Steane code in a trapped-ion architecture [89]. Here, we develop similar schemes based on two-ion MS gates and take into account the specific architectural constraints of our experimental system.

(i) DiVincenzo-Aliferis QEC based on CNOT gates.Figure 10 shows the standard circuit based on CNOT gates for the fault-tolerant measurement of a fourqubit stabilizer. If no error occurs at all, the measurement $M_{2}^{x}$ in the $X$ basis of the second ancilla qubit, which was initially prepared in $\left|x_{+}\right\rangle_{a_{2}}$, will reveal the desired \pm 1 stabilizer information after decoding. Additionally, the remaining ancilla qubits ("check qubits") will end up in the state $|0\rangle_{a_{n}}$ and yield an $M_{1}^{z}=+1, M_{3}^{z}=+1$, and $M_{4}^{z}=+1$ outcome. For this circuit, it can be shown that all dangerous two-qubit errors on the ancilla qubits that propagate to the data qubits, and would induce a logical error, are equivalent to an $X_{a_{3}} X_{a_{4}}$ error. This error, which could result, e.g., from a bit-flip error before the CNOT gate involving ancilla qubits $a_{3}$ and $a_{4}$ during the GHZ-state preparation (encoding), would propagate through two of the CNOT gates in

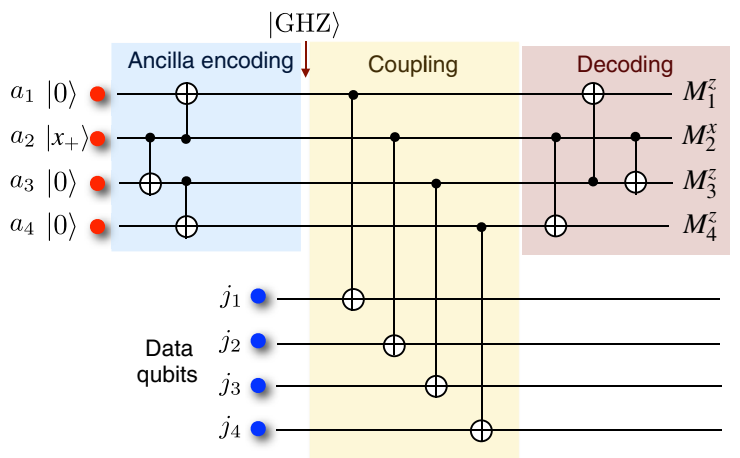

FIG. 10. CNOT-based circuit for DiVincenzo-Aliferis-type QEC: This circuit involves the preparation of an ancilla four-qubit GHZ state by CNOT gates, its transversal coupling to the data qubit layer, the ancilla state decoding, and the measurements of the ancillas. 
the coupling step to the $j_{3}$ and fourth $j_{4}$ qubits. Note, however, that the circuit is constructed in such a way that these two errors, $X_{a_{3}} X_{a_{4}}$, propagate among the ancilla qubits during the decoding stage, causing a bit flip on all three ancilla check qubits and thus yielding $M_{1}^{z}=-1, M_{3}^{z}=-1$, and $M_{4}^{z}=-1$. One can finally show that this outcome on the check ancillas is uniquely associated with the occurrence of such an $X_{a_{3}} X_{a_{4}}$ error. In other words, any single error on a data qubit occurring during the decoding circuit cannot cause the same outcome and thus cannot be confused with the $X_{a_{3}} X_{a_{4}}$ error. Therefore, if the $(-1,-1,-1)$ outcome is obtained, one can correct the two errors by applying $X_{j_{3}} X_{j_{4}}$ to the data qubits, be it physically or on a software bookkeeping level.

(ii) DiVincenzo-Aliferis QEC based on MS gates.Figure 11 displays the detailed circuit for a faulttolerant DiVincenzo-Aliferis-type stabilizer measurement based on two-ion MS gates. The encoding of the four ancilla qubits, i.e., the preparation of the four-qubit GHZ state [see Eq. (28)] by three two-ion MS gates followed by three local Z-type rotations, occurs in the same way as for the DVS scheme, as discussed in Sec. V B 3. Similarly, the coupling to the data qubits takes place transversally by a series of four $X X$ Ms gates and four local $X$ rotations on the

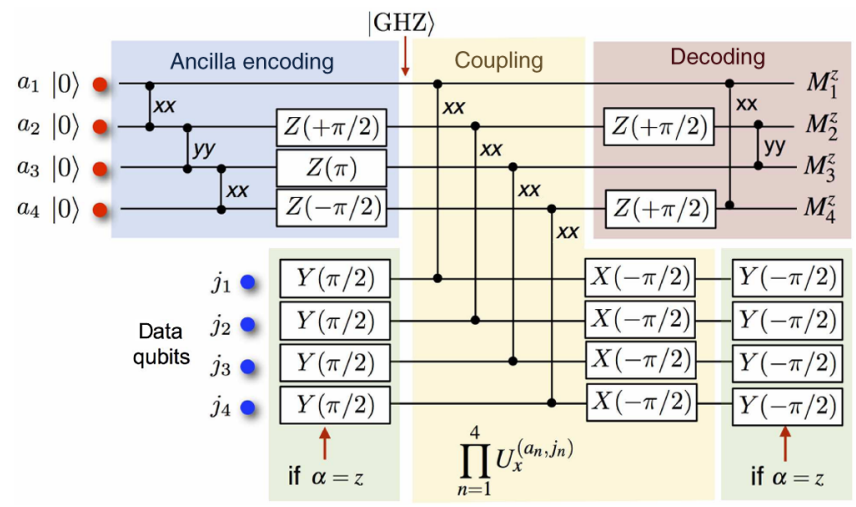

FIG. 11. MS-based circuit for DiVincenzo-Aliferis-type QEC: Initially, a sequence of three two-ion MS gates and local $Z$ rotations is used to prepare the ancilla $|\mathrm{GHZ}\rangle$ [see Eq. (28)]. This state is subsequently coupled transversally by four $X$-type MS gates to the data qubits, thereby mapping the $S_{x}$ stabilizer eigenvalue information onto the relative phase of the GHZ state. The decoding circuit, formed by local $Z$ rotations and two two-ion MS gates, is constructed in such a way that a harmful two-qubit error, which has propagated to the code layer, is unambiguously signaled by the $M_{3}^{z}=+1, M_{4}^{z}=-1$ measurement outcome of the third and fourth ancilla qubits. The measurement of Z-type stabilizers $(\alpha=z)$ is almost identical, also introducing $Y$ rotations of the four data qubits before and after applying the four $X$-type MS gates of the coupling step. data qubits. Before the decoding step, the four ancilla qubits are ideally (if no error has occurred) in the state $(1 / \sqrt{2})\left(\left|x_{+} x_{+} x_{+} x_{+}\right\rangle \pm\left|x_{-} x_{-} x_{-} x_{-}\right\rangle\right)$, depending on whether the data qubits are in $\mathrm{a}+1$ or -1 eigenstate of the measured stabilizer. Under the two $Z$ rotations of Fig. 11, this state is transformed into $Z_{a_{2}}(\pi / 2) Z_{a_{4}}(\pi / 2)(1 / \sqrt{2})\left(\left|x_{+}\right\rangle_{a_{1}} \mid x_{+}\right.$ \rangle$\left._{a_{2}}\left|x_{+}\right\rangle_{a_{3}}\left|x_{+}\right\rangle_{a_{4}} \pm\left|x_{-}\right\rangle_{a_{1}}\left|x_{-}\right\rangle_{a_{2}}\left|x_{-}\right\rangle_{a_{3}}\left|x_{-}\right\rangle_{a_{4}}\right)=:\left|\tilde{\psi}_{3}\right\rangle$, which is a locally equivalent GHZ-type state

$$
\begin{aligned}
\left|\tilde{\psi}_{3}\right\rangle= & \frac{1}{\sqrt{2}}\left(\left|x_{+}\right\rangle_{a_{1}}\left|y_{+}\right\rangle_{a_{2}}\left|x_{+}\right\rangle_{a_{3}}\left|y_{+}\right\rangle_{a_{4}}\right. \\
& \left. \pm\left|x_{-}\right\rangle_{a_{1}}\left|y_{-}\right\rangle_{a_{2}}\left|x_{-}\right\rangle_{a_{3}}\left|y_{-}\right\rangle_{a_{4}}\right) .
\end{aligned}
$$

From this state, it can be readily seen that the subsequent $X$-type MS gate on ancillas $a_{1}, a_{4}$ and the $Y$-type MS gate on ancillas $a_{2}, a_{3}$ decouple the third and fourth qubits, leaving the first two ancilla qubits in one of two possible Bell-type states $Y_{a_{2}, a_{3}}^{2}(\pi / 2) X_{a_{1}, a_{4}}^{2}(\pi / 2)\left|\tilde{\psi}_{3}\right\rangle=\left|\mathrm{B}_{ \pm}\right\rangle \otimes|1\rangle_{a_{3}} \otimes|0\rangle_{a_{4}}$, where

$$
\left|\mathrm{B}_{ \pm}\right\rangle=\frac{1}{\sqrt{2}}\left(\left|x_{+}\right\rangle_{a_{1}}\left|y_{+}\right\rangle_{a_{2}} \mp\left|x_{-}\right\rangle_{a_{1}}\left|y_{-}\right\rangle_{a_{2}}\right) .
$$

Let us now discuss how to access the stabilizer eigenvalue information, simultaneously tracking possible dangerous errors. The first two ancilla qubits being in the Bell-type state [Eq. (30)] are measured, revealing the \pm 1
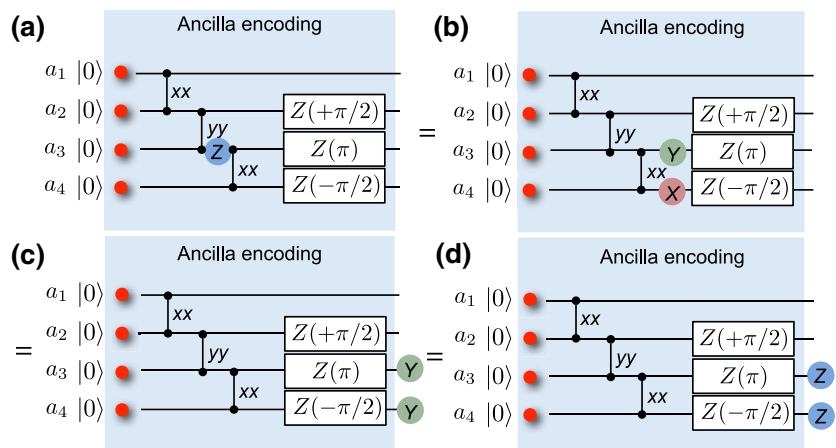

FIG. 12. Dangerous error propagation during the encoding step of the DiVincenzo-Aliferis-type scheme: Subfigures (a)-(d) illustrate how a single phase-flip error $Z_{a_{3}}$, occurring after the $Y Y$ MS gate of the encoding step, results in two phase-flip errors $Z_{a_{3}} Z_{a_{4}}$ that feed into the data qubits. Note that in the last step [from (c) to (d)], we have made use of the fact that a two-qubit $Y_{a_{3}} Y_{a_{4}}$ error is equivalent to $Z_{a_{3}} Z_{a_{4}}$. This can be seen, e.g., by recognizing that the $Y_{a_{3}} Y_{a_{4}}$ error corresponds to simultaneous bit- and phase-flip errors on both qubits, $Y_{a_{3}} Y_{a_{4}} \equiv Z_{a_{3}} Z_{a_{4}} X_{a_{3}} X_{a_{4}}$, and that $X_{a_{3}} X_{a_{4}}$ leaves the ancilla GHZ state $(1 / \sqrt{2})\left(\left|x_{+} x_{+} x_{+} x_{+}\right\rangle+\left|x_{-} x_{-} x_{-} x_{-}\right\rangle\right)$ invariant. 


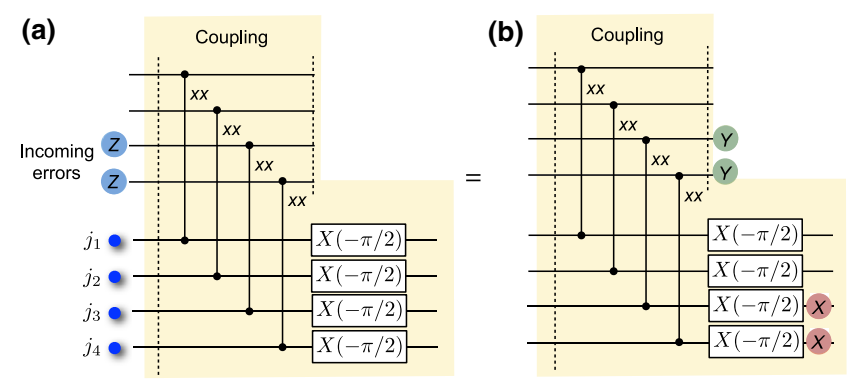

FIG. 13. Dangerous error propagation during the coupling step of the DiVincenzo-Aliferis-type scheme: The circuits show how an incoming $Z_{a_{3}} Z_{a_{4}}$ error, resulting, e.g., from a single phase-flip error in the encoding step in Fig. 12, is converted into a pair of $Y$-type errors on the ancilla qubits and furthermore propagate into the data qubit layer, where they result in two bit-flip $X_{j_{3}} X_{j_{4}}$ errors.
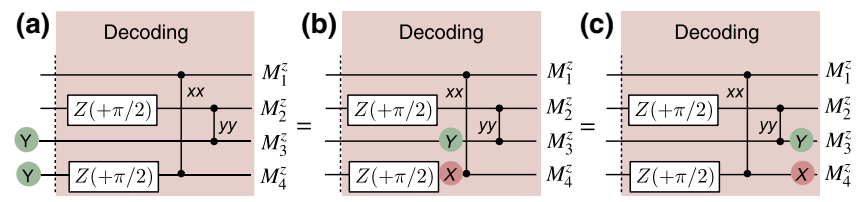

FIG. 14. Detection of the dangerous two-qubit errors in the DiVincenzo-Aliferis-type scheme: The circuits show how an incoming $Y_{a_{3}} Y_{a_{4}}$ error, resulting from the transversal-coupling step in Fig. 13, results in bit flips $\left(Y_{a_{3}}\right.$ and $\left.X_{a_{4}}\right)$ on the third and fourth ancilla qubits. However, in this case, the third and fourth qubits end up in $|0\rangle_{a_{3}}$ and $|1\rangle_{a_{4}}$, respectively, which results in an $M_{3}^{z}=+1, M_{4}^{z}=-1$ measurement outcome.

stabilizer eigenvalue by the odd or even parity of the outcome $\left(M_{1}^{z}, M_{2}^{z}\right)$. The third and fourth ancilla qubits act as checks and are expected to yield $M_{3}^{z}=-1$ and $M_{4}^{z}=+1$ in the ideal case without any errors. Similar to the case of the DVS scheme discussed above, it can be shown that all dangerous two-qubit errors in the circuit of Fig. 11 are equivalent to a $Z_{a_{3}} Z_{a_{4}}$ error at the end of the encoding circuit. One example of such dangerous error histories is shown in Figs. 12-14 for the encoding, transversal coupling, and decoding steps of the DVAtype scheme, respectively. As shown in these figures, this type of $Z_{a_{3}} Z_{a_{4}}$ error (see Fig. 12), which has propagated to two $X_{j_{3}} X_{j_{4}}$ errors in the code layer (see Fig. 13), is unambiguously signaled by the $M_{3}^{z}=+1$, $M_{4}^{z}=-1$ outcome of the third and fourth ancilla qubits (see Fig. 14).

\section{TRAPPED-ION PROTOCOLS TO ASSESS THE BREAKEVEN POINT FOR BENEFICIAL QEC}

Based on the description of the experimental capabilities in Sec. III, the development of an effective error model composed of different quantum channels in Sec. IV, and the trapped-ion toolbox for QEC in Sec. V, we can now present the different trapped-ion protocols to assess the breakeven point for beneficial QEC [Eqs. (2) and (3)] in the sevenqubit color code. This code, being equivalent to the sevenqubit Steane code [5], has been subjected to a series of studies assessing its QEC performance and error thresholds (we refer the reader to Ref. [90] for a comparative study of this code and other small-scale QEC codes). Depending on the modeling of the noise, typical error thresholds lie around the $10^{-4}$ level $[90,91]$. Additionally, we remark that previous work has also explored the performance of this code in a trapped-ion architecture [89], considering CNOTgate and shuttling-based protocols in a two-dimensional array of coupled traps, paying particular attention to the influence of available ancilla resources.

As mentioned in the introduction, the QEC protocols that we explore in this work exploit optimized MS-gate-based circuitry and are embedded in a single, segmented linear trap. The various protocols we investigate are based on either (i) spectroscopic decoupling, i.e., storing idling qubits temporarily in Zeeman sublevels that do not belong to the computational subspace, or (ii) ion reconfiguration, i.e., combination of shuttling, splitting, merging, and rotations of the ion crystal to physically move idle qubits to the storage region. For all the different protocols within these two types, we have developed three conceptual layers:

(1) Real-space representation.- A sketch of the sequence of operations in real space and time, which is particularly useful to visualize the effect of ion-crystal reconfigurations in the shuttling-based protocols.

(2) Circuit representation. - Circuit diagrams that show the entirety of elementary operations that should be applied in the real experiment. Besides the gate operations, measurements, etc., these contain a list of the ion-crystal-reconfiguration building blocks (splitting, shuttling, merging, and rotation operations).

(3) Quantum-channel representation.- Circuit diagrams containing information about the sequence of quantum channels, which reflect the effective noise models used to describe imperfect operations in Sec. IV, as well as the dependence of channel parameters on the experimentally relevant metrics (e.g., gate times and infidelities, coherence times). This underlies the numerical simulations of the following section.

In the following, we describe in detail these three layers for the first shuttling-based protocol and restrict to the realspace representation for the remaining ones.

\section{A. Non-fault-tolerant trapped-ion QEC protocols}

In this section, we discuss the protocols for a QEC cycle with an eight- or nine-ion crystal, where seven data qubits are used to implement the aforementioned seven-qubit 
color code, and one additional ancillary qubit is exploited for syndrome extraction by measuring the six stabilizers in Eq. (23) (see Fig. 3). For one of the protocols, we require an extra ion to implement laser cooling of the ion crystal. By using a single ancillary qubit, this QEC protocol cannot be made fault tolerant (see Sec. V B 2). However, even in this case, it is very instructive to use it to benchmark the experimental progress towards fault-tolerant quantum computation according to the criteria in Eqs. (2) and (3). As shown in Sec. VII, one could already prove the nontrivial beneficial character of our QEC protocol by improving the experimental hardware according to Tables II and IV. Moreover, this is the less-demanding possible scenario for future trapped-ion experiments along the lines of Ref. [15].

We consider both shuttling- and hiding-based approaches, as well as stabilizer mappings based on either multiqubit MS gates (cf. Fig. 5) or on a sequence of twoqubit MS gates (cf. Fig. 6). The criteria for beneficial QEC exposed in Eqs. (2) and (3) of Sec. II can be assessed through the quantum-information protocols detailed in Table I. In order to implement this protocol numerically, we now present the explicit scheduling of different shuttling- and hiding-based approaches for a single QEC cycle in a trapped-ion seven-qubit color code.

\section{Shuttling-based, single-species, multiqubit gate protocol}

Let us start by considering a shuttling-based approach to the multiqubit mapping of each of the data-qubit stabilizers onto the ancillary qubit (see Fig. 5), both of which belong to the same atomic species. Since all the ions in this protocol are of the same species, and the lasers responsible for the entangling MS gates [Eq. (4)] act globally on the ion chain, one needs to combine storage and processing or manipulation zones in the trap, and reconfigure the ion crystal in order to sequentially apply the readout of all six stabilizers of the code [Eq. (23)].

In Fig. 15, we depict the real-space representation of this protocol, which utilizes a single arm of the segmented ion trap of Fig. 1. In this case, we only make use of two storage zones, $S_{1}$ and $S_{2}$, surrounding a central manipulation region $M_{2}$, within one arm of the trap. Initially, Alice encodes an arbitrary state $|\psi\rangle=\alpha|0\rangle+\beta|1\rangle$ in the seven data qubits and hands them to Bob, who can ask his assistant Igor to perform an imperfect round of QEC before he decides if the original state was either $|\psi\rangle$ or $\left|\psi_{\perp}\right\rangle=\beta^{*}|0\rangle-\alpha^{*}|1\rangle$. In this protocol, Igor has an additional ancillary ion of the same species, which can be used for the readout of the stabilizer information. He distributes the seven data qubits within the two storage zones forming two separate ion crystals and locates the ancillary ion in the manipulation zone (see Fig. 15). The steps of such a QEC cycle are described by different operations in the figure (time evolution occurs downwards), in which these crystals are split, such that the

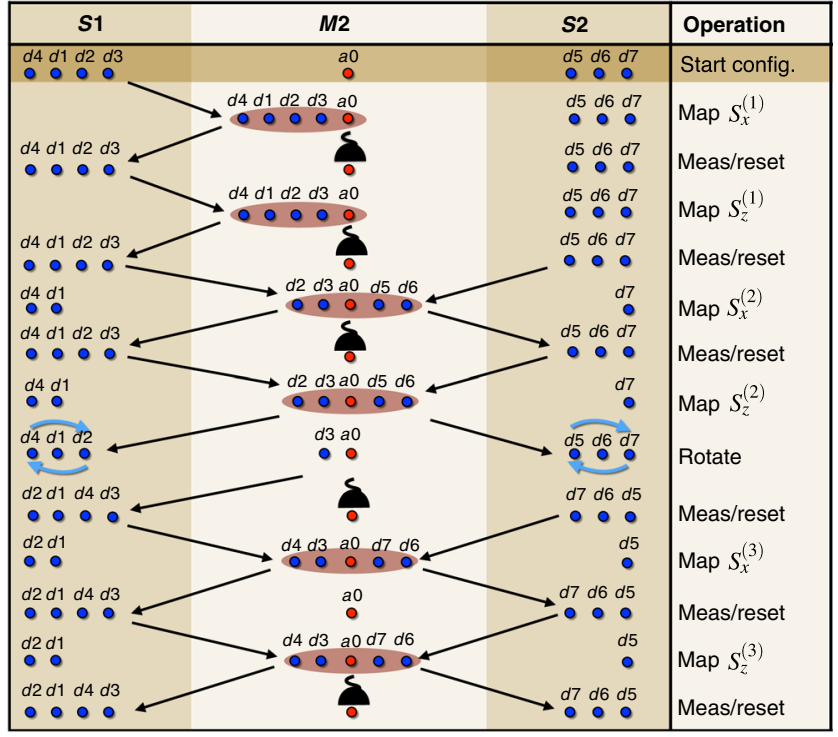

FIG. 15. Real-space representation of shuttling-based onespecies QEC cycle with multiqubit MS gates: Data and ancillary ions are represented by blue and red dots, respectively, and are distributed within the storage and manipulation regions of the first row. The black arrows pointing to the storage zone represent splitting operations followed by a shuttling of a subset of physical ions onto the processing zone, after which they all merge with the static ancillary ion. The black arrows towards the storage zones represent splitting operations, followed by a shuttling of the physical ions back to the storage zone, where they are merged with any physical ions that might already be present there. The blue arrows within the storage zone represent crystal rotations that reorder the physical ions. On the right column, we specify the times where the stabilizer mappings Map $S_{\alpha}^{(m)}$, implemented by multiqubit entangling gates and represented by red ellipses, and ancillary measurements or reset (Meas or reset), represented by a black detector, are applied.

data qubits of a given stabilizer can be shuttled to the manipulation region and merged with the ancillary ion. At this stage, Igor applies the stabilizer mapping in Fig. 5 by shining the corresponding lasers onto the ions of the manipulation zone. After splitting the chain and shuttling the data qubits back to the storage zone, the ancillary ion can be measured by state-dependent fluorescence, such that Igor can collect the syndrome information. Note that the scattered photons do not affect the information stored in the physical qubits, as these have been shuttled to the distant storage regions.

These steps must be repeated for each of the stabilizers [Eq. (23)] of the seven-qubit color code. As depicted in Fig. 15, the last two stabilizer mappings require a reordering of the ion crystals in the storage zones, which can be accomplished by rotating the crystal, effectively implementing a mirror image about the symmetry axis of the code, such that the roles of plaquettes 2 and 3 are interchanged (see Fig. 3). In this way, one can repeat the same crystal-reconfiguration operations of the second set of 

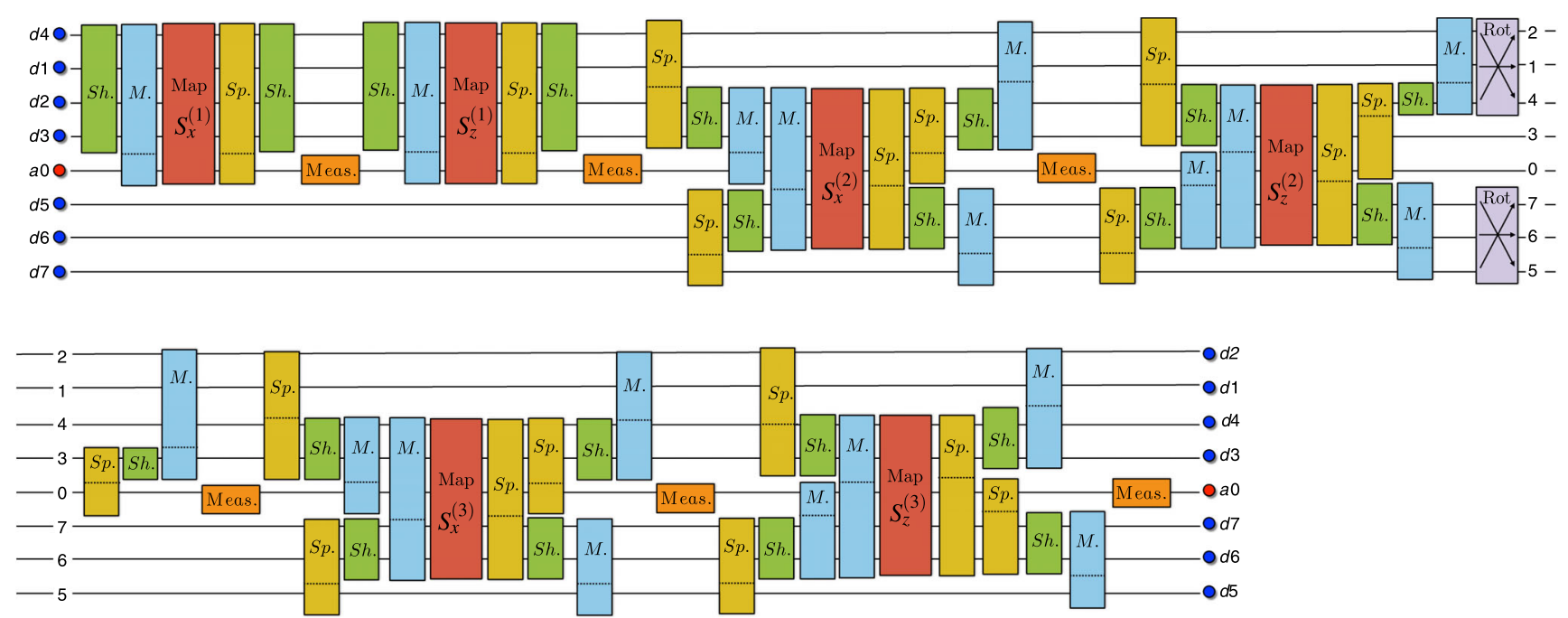

FIG. 16. Circuit representation of shuttling-based one-species QEC cycle with multiqubit MS gates: The data $\left\{d_{1}, \ldots, d_{7}\right\}$ and ancillary $a_{0}$ ions are arranged vertically, and a set of boxes represents the elementary operations taking place at a particular time step: "Sh." stands for shuttling of the ions within the green boxes, "M." stands for the merging of the two sets of ions separated by a dotted line within the blue boxes, "Sp." stands for splitting of the crystal into two sets of ions separated by a dotted line within the yellow boxes, "Meas." stands for the ancillary ion measurement in the orange boxes, and "Rot." stands for the rotation of the ion crystal within the purple boxes, with arrows representing the corresponding crystal reordering. Note that some crystal-reconfiguration operations, such as merging or splitting and shuttling, can be operated simultaneously in different trap zones. Finally, Map $S_{\alpha}^{(m)}$ stands for the mapping of a particular stabilizer $S_{\alpha}^{(m)}$ involving the data and ancillary ions within the red boxes. These red boxes contain the sequence of quantum gates in Fig. 5.

stabilizers and finalize the syndrome extraction. We note that similar combinations of rotations and split, shuttler or merge operations will be a crucial building block of all shuttling-based QEC protocols in the following sections.

In Fig. 16, we describe the circuit representation of this protocol, where one can keep track of the sequential order of the different elementary operations (time evolving from left to right in this figure). At each time step, a given box describes which operation takes place on which set of ions (see the caption of Fig. 16 for a detailed account).

In order to explore the performance of Igor's QEC cycle under realistic experimental errors and sources of noise, we need to consider the time intervals during which these operations take place, as idle qubits will be subjected to a dephasing noise [Eq. (13)] that degrades the information stored in the code. Moreover, we also need to consider that the ion reconfigurations excite the motional modes of the ion crystal, affecting the fidelity of the MS gates [Eq. (17)] in subsequent stabilizer mappings, which enter in the depolarizing channels of Eqs. (14)-(16). This goes beyond standard noise models in the literature, all of which assume that the gate errors are nonincreasing with the depth of the circuit. Finally, we need to take into account that the measurement and reset of the ancillary ion are also faulty, which is accounted for by the bit-flip channel [Eq. (22)]. Accordingly, the circuit representation is translated into the quantum-channel description of Fig. 17, where the above errors are represented as particular Markovian error channels (see Sec. IV) with error probabilities that depend on the history of previous operations and on the total time required for each of the steps (see the caption of Fig. 17 for details). This circuit of ideal gates, interspersed with dephasing, depolarizing, and bit-flip channels, is numerically simulated in Sec. VII to estimate the breakeven point that determines when the QEC cycle becomes useful.

\section{Shuttling-based, two-species, multiqubit gate protocol}

Let us now reconsider the same QEC cycle with a twospecies ion crystal, where the seven data qubits and the additional ancillary qubit are of a different atomic species. This has two important implications: (i) It reduces the number of crystal reconfigurations that must be implemented by Igor since the measurement of the ancillary qubit does not need to be performed on an isolated qubit. Even if the physical ions are in the same processing region as the ancilla ion, the photons that are scattered while the ancilla ion is being measured will not be absorbed by them, and thus the encoded state will not be affected. On the other hand, the photon recoil onto the ion crystal can induce motional excitations that would compromise the fidelities of subsequent MS entangling gates. However, (ii) the use of two species allows Igor to apply sympathetic recooling of the ion crystal, which minimizes the number of motional excitations due to recoil or crystal reconfigurations prior to any MS gate in the stabilizer mappings and thus reduces the motional error [Eq. (17)] during the entangling gates. 


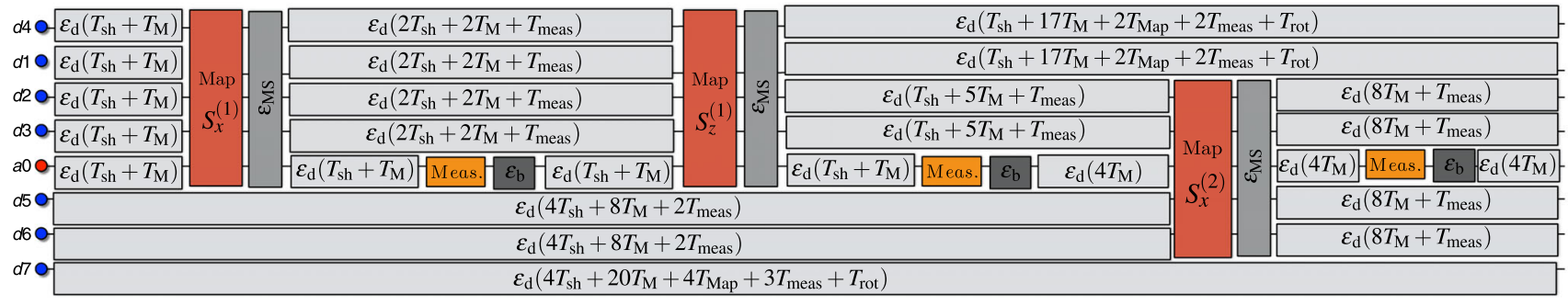

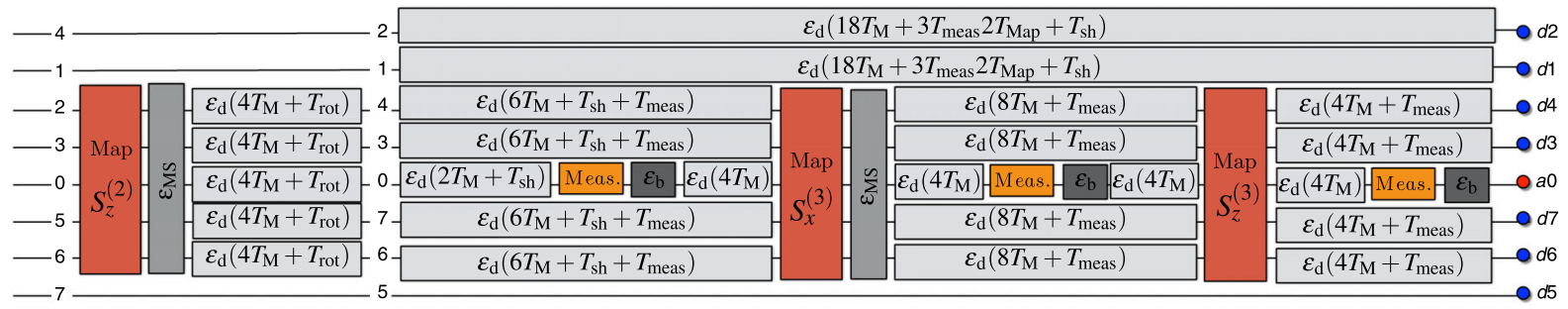

FIG. 17. Quantum-channel representation of shuttling-based one-species QEC cycle with multiqubit MS gates: The ion reconfiguration steps of Fig. 16 lead to an increase of the phonon populations and a dephasing of the idle qubits during the time required for these reconfigurations to take place. Additionally, idle ions also dephase during the time lapse of the stabilizer mapping. These time intervals are $T_{\text {sh }}$ for shuttling, $T_{M}$ for merging or splitting, $T_{\text {Map }}$ for stabilizer mapping, $T_{\text {meas }}$ for measuring, and $T_{\text {rot }}$ for rotations. The dephasing channel applied during a certain period is depicted by light grey boxes, with the channel $\varepsilon_{\mathrm{d}}\left(n_{\mathrm{sh}} T_{\mathrm{sh}}+n_{M} T_{M}+n_{\text {Map }} T_{\text {Map }}+n_{\text {meas }} T_{\text {meas }}+\right.$ $n_{\text {rot }} T_{\text {rot }}$ ) acting on a particular qubit, where $n_{o}$ is the number of operations of the type $o$ that occur within that period. The actual channel corresponds to Eq. (13) with a probability $p_{d}=\sum_{o} n_{o} T_{o} / 2 T_{2}$, with $T_{2}$ being the coherence time of the qubits. The ancilla readout and reset measure is modeled by a bit-flip channel (22) acting on the ancillary qubit $\varepsilon_{b}$ with a probability given by the sum of the measurement and reset errors $p_{b}=\varepsilon_{\text {meas }}+\varepsilon_{\text {res }}$, as reported in Table II, and represented by dark grey boxes after each measurement. Finally, map is modeled by an ideal stabilizer mapping acting on the particular set of qubits, followed by a depolarizing channel $\varepsilon_{\mathrm{MS}}$ (i.e., grey box) given by one of the three channels of Eqs. (14)-(16) with an error probability $p_{d}=\varepsilon_{m}+\varepsilon_{d}+\varepsilon_{I}$ that depends on the current phonon number through Eq. (17), and the gate time via Eqs. (19) and (20).

The real-space representation of this two-species protocol is depicted in Fig. 18. As mentioned previously, the number of crystal-reconfiguration operations is highly reduced with respect to the one-species protocol in Fig. 15. Additionally, intermediate recooling steps can also be introduced before any stabilizer mapping, such that the fidelity of the corresponding MS entangling gates is not compromised as the QEC cycle proceeds. The circuit and quantum-channel representations are somewhat similar to those of the one-species protocol in Figs. 16 and 17, and will not be presented in detail. However, we note that they are also important, as they provide a scheduling of the physical operations that would have to be applied in an experiment, and they also contain the relevant information for the numerical modeling of Sec. VII.

\section{Shuttling-based, two-species, two-qubit gate protocol}

We now consider a similar shuttling-based, two-species QEC protocol, but we rely on sequential two-qubit MS gates for the stabilizer mapping (see Sec. V B 2). We recall that using the combination of a MS gate between a pair of ancilla $a_{0}$ and data $d_{j}$ qubits, followed by a rotation on the data qubit, one can implement the conditional gates $U_{x}^{\left(a_{0}, d_{j}\right)}$ in Eq. (25). The sequential combination of these gates, together with possible rotations on data qubits
$Y_{d_{j}}( \pm \pi / 2)$, leads to a mapping of the stabilizer information into the ancilla qubit (see Fig. 6), which can be obtained from its fluorescence $M_{0}^{z}$.

In order to reduce the complexity of all the crystal reconfigurations required to perform the sequential gates of the QEC cycle, Igor makes use of all five regions of the segmented trap in Fig. 1 (i.e., two manipulation zones $M_{1}$ and $M_{2}$ interspersed between three storage regions $S_{1}, S_{2}$, $S_{3}$ ). Moreover, since the ancilla qubit is not measured after each of the conditional gates $U_{x}^{\left(a_{0}, d_{j}\right)}$, Igor needs to recool the crystal several times during each stabilizer readout without affecting the ancilla and data qubits. Therefore, we consider that the ancilla and data ions are of the same species, but we equip Igor with an extra ion of a different species $c$ for sympathetic recooling of the crystal. Hence, Igor has $7+1+1$ ions for his imperfect round of QEC, which he distributes within the segmented trap as depicted in the starting configuration of Fig. 19. The data qubits of a given stabilizer are shuttled one by one from the storage regions onto the manipulation zone $M_{2}$, where sympathetic recooling represented by blue rectangles is applied prior to the data-ancilla mapping $U_{x}^{\left(a_{0}, d_{j}\right)}$ or $Y_{d_{j}}(+\pi / 2) U_{x}^{\left(a_{0}, d_{j}\right)} Y_{d_{j}}(-\pi / 2)$ for an $X$ - or $Z$-type stabilizer readout, labeled as $\operatorname{Map}\left(a_{0}, d_{j}\right)$ and represented by red ellipses. After all the four data qubits of a particular 


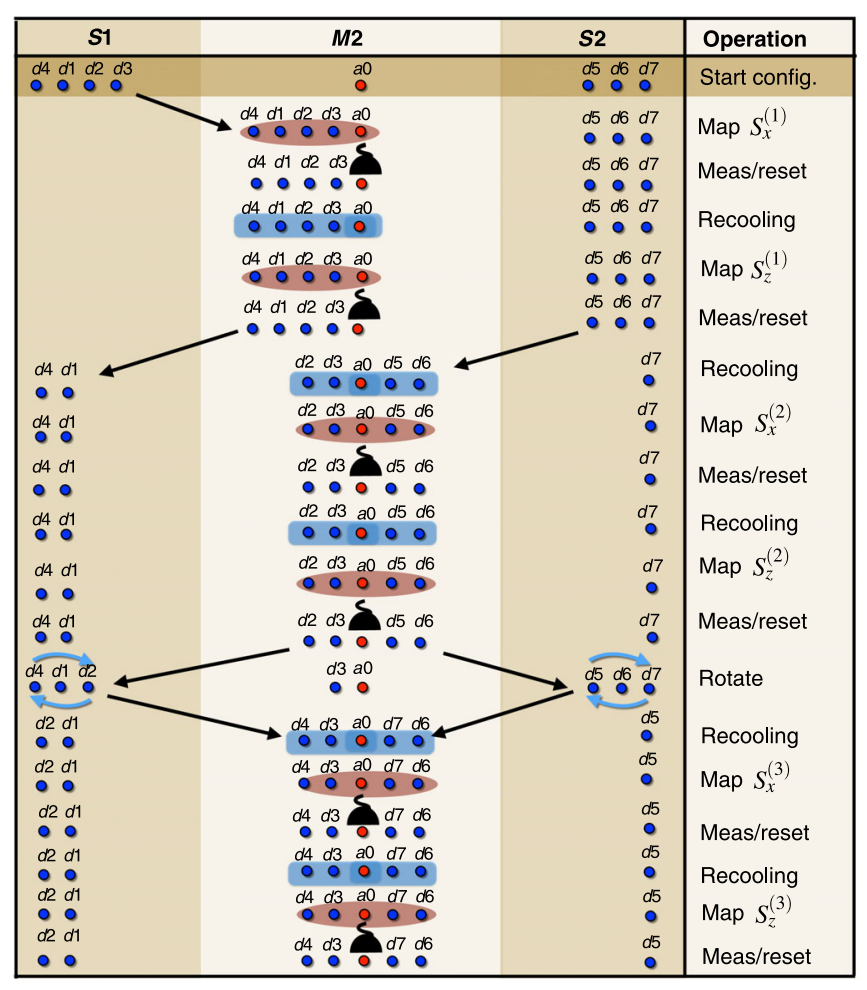

FIG. 18. Real-space representation of shuttling-based, twospecies QEC cycle with multi-qubit MS gates: We use the same conventions as in Fig. 15. As announced in the main text, the number of crystal reconfigurations is reduced with respect to the shuttling-based one-species protocol. In the rightmost column, we specify the times when crystal rotations, stabilizer mappings, ancillary measurements, and the new sympathetic (Recooling), represented by blue rectangles, are applied.

stabilizer have been coupled to the ancilla ion, Igor can proceed to isolate the ancilla-cooling pair of ions in the manipulation zone and collect the state-dependent fluorescence of the ancilla ion, in this way inferring the stabilizer information.

As can be seen in this figure, the complexity of the crystal-reconfiguration operations increases considerably, such that idle qubits will suffer more environmental dephasing during the QEC cycle. Therefore, although the multiqubit errors [Eq. (16)] of the collective stabilizer mapping of the previous subsections are avoided in this scheme, and the fidelity for two-qubit MS gates is higher than that of five-multiqubit ones (see Table II), we do not expect any big improvement of the non-fault-tolerant protocol. A possible advantage can only take place if a fully fault-tolerant scheme is implemented.

\section{Hiding-based, two-species, multiqubit gate protocol}

An alternative approach to the shutting-based protocols presented above is to work with a static ion crystal but equip Igor with spectroscopic decoupling capabilities. Accordingly, Igor can take a particular subset of ions from a given stabilizer mapping by spectroscopically decoupling

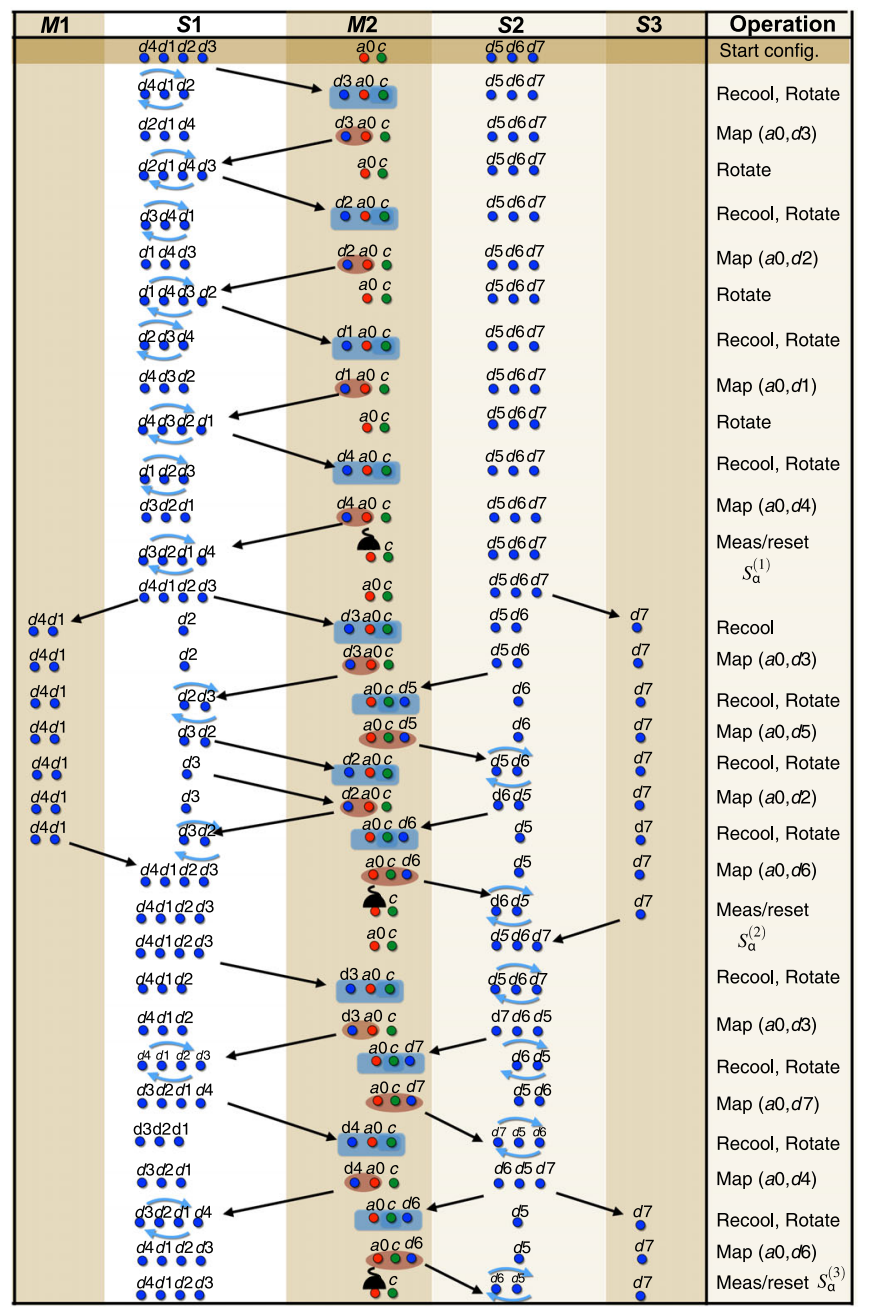

FIG. 19. Real-space representation of shuttling-based twospecies QEC cycle with two-qubit MS gates: We represent half of a cycle of QEC for the sequential measurement of $\left\{S_{\alpha}^{(1)}, S_{\alpha}^{(2)}, S_{\alpha}^{(3)}\right\}$, either for $\alpha=x$ or for $z$, and use the same conventions as in Figs. 15 and 18, placing the extra cooling ion (green circle) in the manipulation zone $M_{2}$. In the rightmost column, we specify the time steps when crystal-reconfiguration operations, sympathetic recooling, and ancilla measurement/reset take place. The new mapping functions, represented by red ellipses, consist of the data-ancilla conditional gate $\operatorname{Map}(a 0, d j)=U_{x}^{\left(a_{0}, d_{j}\right)}$ for a $X$-type stabilizer or its combination with local rotations $\operatorname{Map}(a 0, d j)=Y_{d_{j}}(+\pi / 2) U_{x}^{\left(a_{0}, d_{j}\right)} Y_{d_{j}}(-\pi / 2)$ for a $Z$-type stabilizer. We recall that these local rotations can be obtained from the available set of gates in Eqs. (5) and (6) by simple refocusing sequences $Y_{d_{j}}(\theta)=$ $U_{\mathrm{R}, \pi / 2}(\theta / 2) U_{\mathrm{R}_{d_{j}}, z}(\pi) U_{\mathrm{R}, \pi / 2}(-\theta / 2) U_{\mathrm{R}_{d_{j}}, z}(-\pi)$.

them and coherently shelving the physical qubit's population in electronic states that do not couple to the lasers that drive the gate operations on the qubit transition (see also Secs. III B and IV C).

We consider the scenario of a two-species encoding. This allows one to measure the ancilla qubit after the stabilizer 


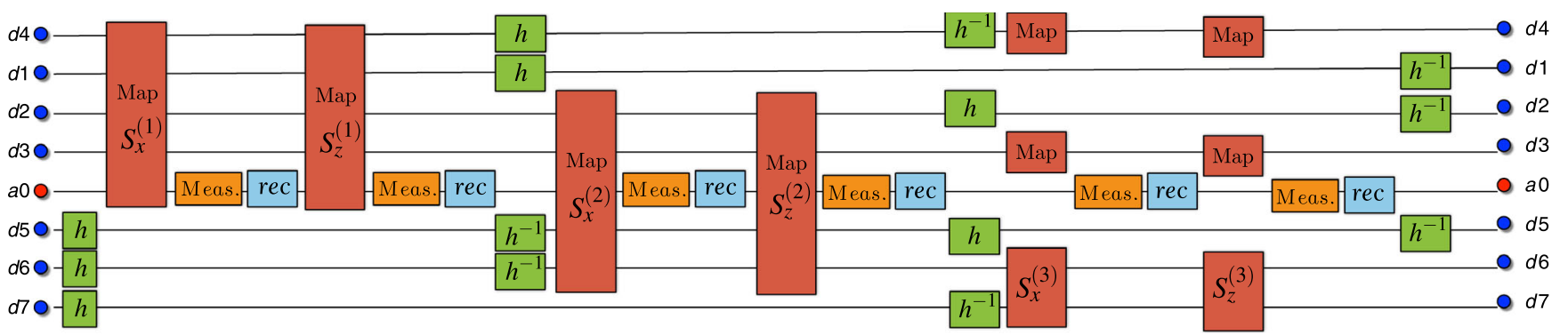

FIG. 20. Circuit representation of the hiding-based, two-species QEC cycle with multiqubit MS gates: We use the same conventions as in Fig. 16. The additional hiding $h$ and unhiding operations $h^{-1}$, represented by green boxes, are realized by composite pulse sequences to coherently map the state of physical qubits from $4 S_{1 / 2}\left(m_{f}=-1 / 2\right)$ and $3 D_{5 / 2}\left(m_{f}=-1 / 2\right)$ to a set of storage $D$ states (see Sec. III C).

mapping, without affecting the state of nonhidden data qubits. In addition, the ancilla qubit, encoded in an ion species that is different from the data qubits, enables recooling of the entire ion string after each fluorescence ancilla measurement, thus minimizing motional excitations due to photon recoil.

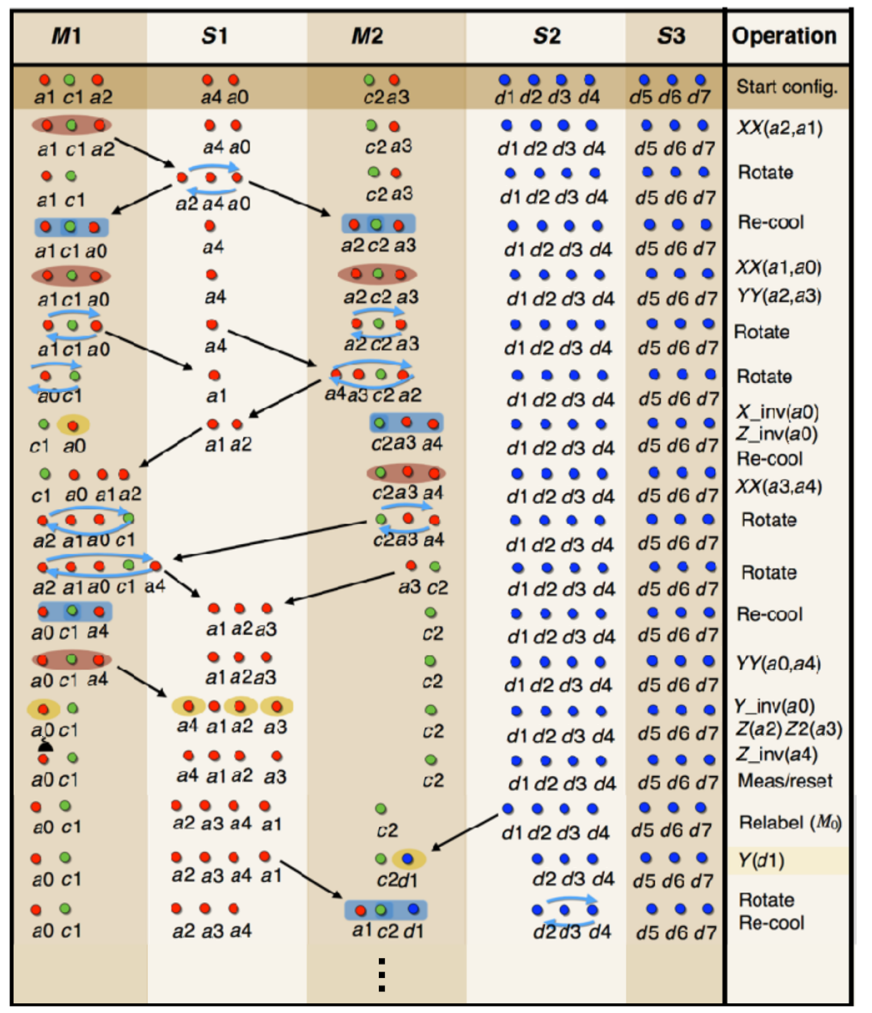

The scheme for a complete QEC cycle involves an overall number of 12 hiding and unhiding operations, each of which can be realized by a composite pulse sequence (nine single-ion pulses detailed in Sec. III C) that maps the state of data qubits to a set of storage $D$ states coherently [15]. Rather than displaying the real-space configuration, it

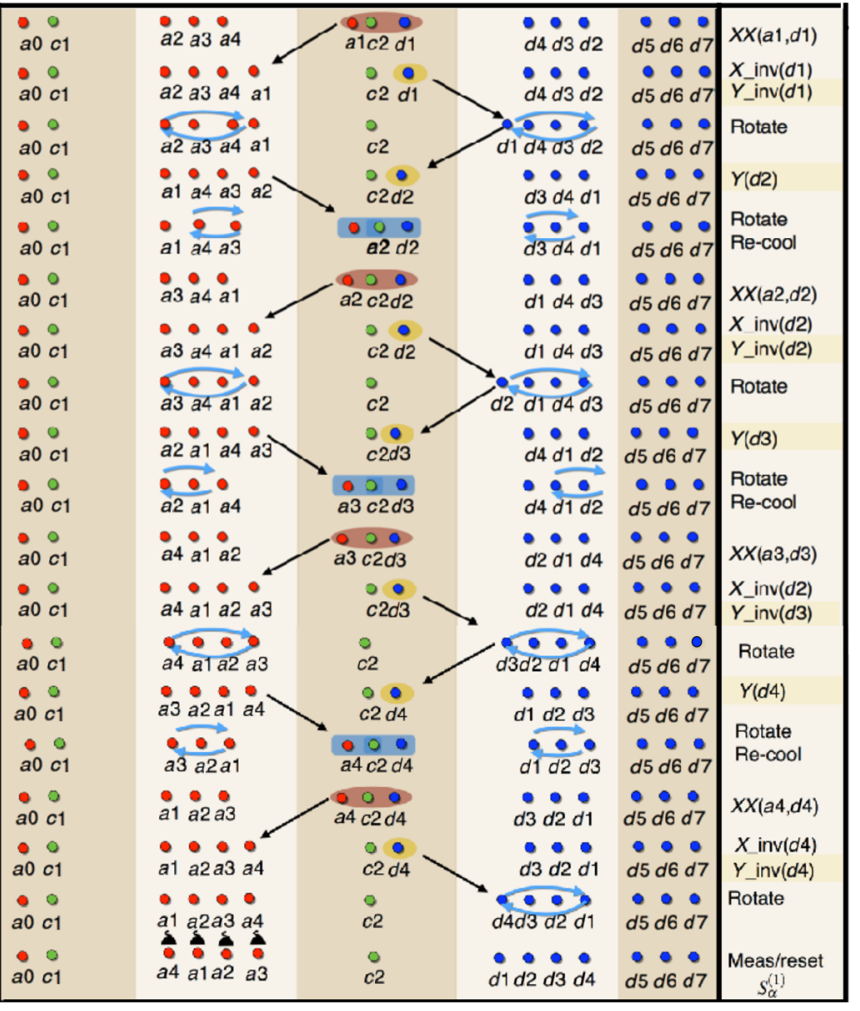

FIG. 21. Real-space representation of the shuttling-based, two-species QEC cycle with a DiVincenzo-Shor scheme: We represent the sequence of operations for the fault-tolerant DiVincenzo-Shor readout of a single stabilizer operator $S_{\alpha}^{(1)}$ for $\alpha=x$ or $z$ in two panels, and we use the same conventions as in Figs. 15, 18, and 19. The extra cooling ions $\mathrm{c} 1, \mathrm{c} 2$ are placed in the manipulation zones $M_{1}, M_{2}$, and are again depicted by a green circle. The rightmost columns of both panels contain the sequence of operations that take place. In addition to the ones described in previous figures, we include $X$ - and $Y$-type Ms gates $X X(i, j)$ and $Y Y(i, j)$ depicted by red ellipses, as well as single-qubit rotations $X(j), X \_$inv $(j)$ corresponding to $X_{j}( \pm \pi / 2)(7)$, and analogously for $Y(j), Y \_$inv $(j)$, and $Z(j), Z \_$inv $(j)$, all of which are depicted by yellow ellipses. We also introduce $Z 2(j)$, which corresponds to $Z_{j}(\pi)$. Finally, $\operatorname{some} Y(j), Y$ inv $(j) \operatorname{rotations}$ on the rightmost columns of each panel are shown inside a yellow rectangle, which implies that they are only applied for a $Z$-type stabilizer readout $(\alpha=z)$. We have also included a classical relabeling operation, Relabel, conditional on the measurement outcome $M_{0}^{z}$. 
is more instructive in this case to give the circuit representation since no crystal reconfiguration is used and all ions reside in the same manipulation zone. In Fig. 20, green boxes depict the hiding or unhiding pulses, which determine which qubits are involved in the respective stabilizer represented by red boxes. Idle qubits undergo dephasing with a strength according to the time durations of the respective mapping, measurement, recooling, and (un) hiding operations acting on the other qubit(s).

\section{B. Fault-tolerant, trapped-ion QEC protocols}

In this section, we discuss the trapped-ion protocols for fault-tolerant QEC with the seven-qubit color code. As emphasized in previous sections, we need to go beyond a single ancillary qubit by focusing on either the DVS or DVA schemes described in Secs. V B 3 and V B 4, respectively. We consider only shuttling-based approaches since the number of spectroscopic decoupling pulses for a hiding-based scheme would increase dramatically for these fault-tolerant protocols. Therefore, a fully hiding-based fault-tolerant protocol with a static ion crystal is likely to perform worse than the shuttling-based alternatives.

\section{Shuttling-based, two-species, DiVincenzo-Shor scheme}

We first consider the trapped-ion implementation of the DVS scheme, which combines the ancilla encoding and verification of Fig. 8 with the subsequent transversal coupling to data qubits of Fig. 9 for each of the code stabilizers. To implement a cycle of QEC using this scheme, Igor must be equipped with five additional ancillary ions $a_{0}, \ldots, a_{4}$. Furthermore, to simplify all of the required crystal reconfigurations, we equip Igor with a couple of cooling ions $c_{1}, c_{2}$ of a different atomic species such that sympathetic recooling can be implemented prior to any entangling MS gate. Igor distributes the seven data qubits, together with the ancillary and cooling ions, according to the starting configuration of Fig. 21. In the left panel of this figure, prior to the ancilla measurement $M_{0}^{z}$, we depict the different operations that Igor must apply for the ancilla encoding and verification. If this part of the protocol is successful, $M_{0}^{z}=+1$, one relabels the ancillary qubits and proceeds with the rest of the scheme. The set of instructions that follow this relabeling corresponds to the transversal coupling to the data qubits of Fig. 9 and ends in the measurement of the remaining ancillas $\left(M_{1}^{z}, M_{2}^{z}, M_{3}^{z}, M_{4}^{z}\right)$, the parity of which allows Igor to infer the $S_{\alpha}^{(1)}$ stabilizer information. After reordering of the data qubits to move the ions of another stabilizer to the storage region $S_{2}$, one can repeat the same procedure in Fig. 9 for the next stabilizer and proceed to complete a full round of QEC. Note that most of these reordering operations can be implemented during the measurement period such that no extra dephasing occurs. To take advantage of the faulttolerant nature of the scheme, Igor should run two full rounds of stabilizer readout. If the results coincide, he should then apply a decoder to determine which error has occurred and apply a particular single-qubit $X$ - or $Z$-type rotation to the corresponding data qubit to correct for it.

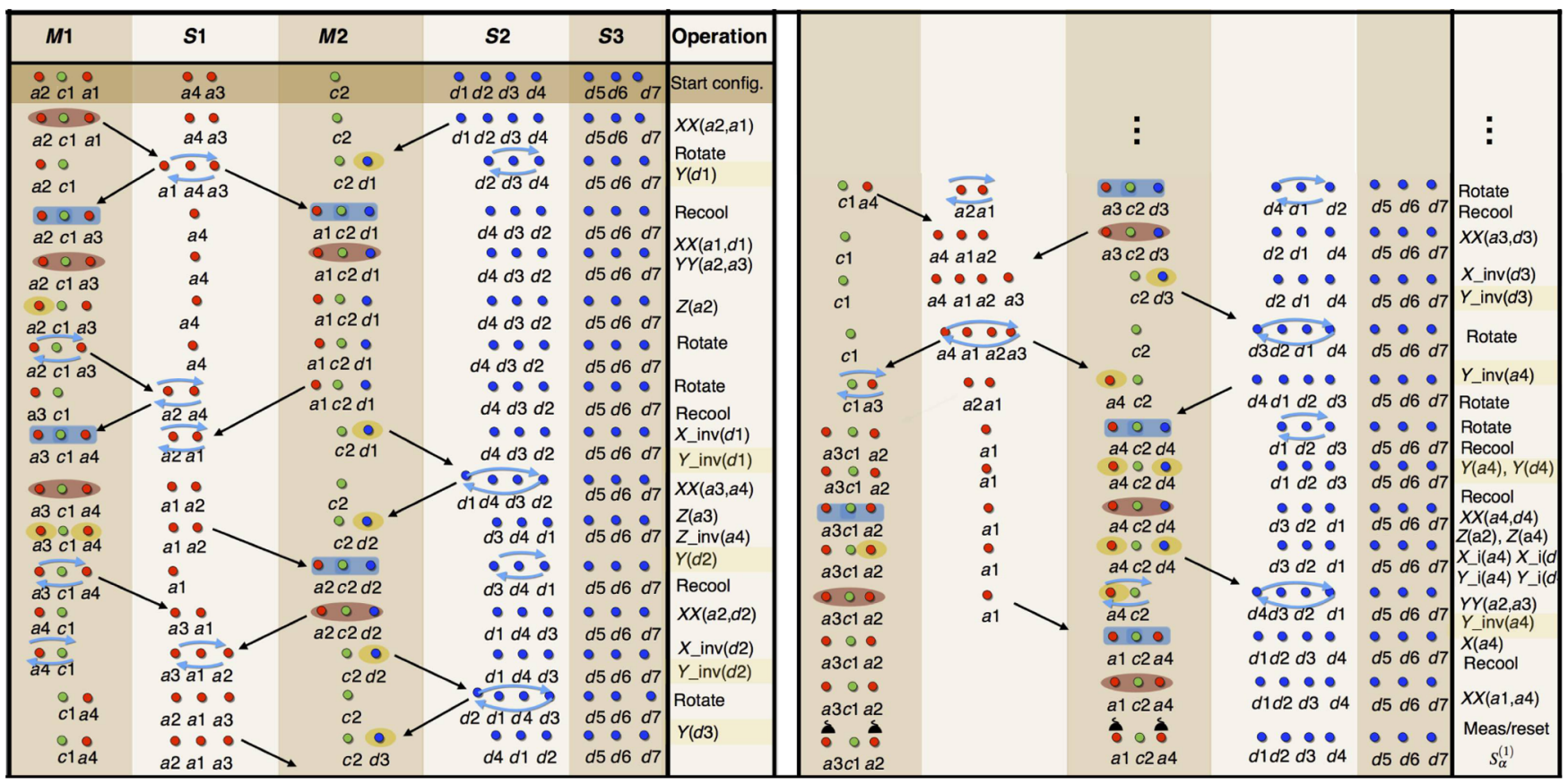

FIG. 22. Real-space representation of the shuttling-based, two-species QEC cycle with a DiVincenzo-Aliferis scheme: We represent the operations for the fault-tolerant DiVincenzo-Aliferis readout of a single stabilizer operator $S_{\alpha}^{(1)}$, either for $\alpha=x$ or $z$, in two panels, and we use the same conventions as in Figs. 15, 18, 19, and 21. 
If the measurement results do not match, Igor should apply the full readout scheme once more and use the decoder on the third set of stabilizer values.

\section{Shuttling-based, two-species, DiVincenzo-Aliferis scheme}

Let us now describe the trapped-ion implementation of the DVA scheme in which the ancilla encoding, coupling, and verification must occur along the prescription of Fig. 11. In this case, it suffices to equip Igor with four additional ancillary ions $a_{1}, \ldots, a_{4}$ and a couple of cooling ions $c_{1}, c_{2}$ of a different atomic species. The distribution of these ions within the different zones of the segmented trap is specified in the real-space representation of Fig. 22, where we also list the operations that Igor must perform for the readout of the $S_{\alpha}^{(1)}$ stabilizer. In contrast to the DVS scheme, the verification step takes place in the final measurement step and depends on the outcome $\left(M_{3}^{z}, M_{4}^{z}\right)$ of the check ancilla qubits. If Igor obtains $(+1,-1)$, this signals that two errors have propagated into the code space, and Igor must apply $X$ - or $Z$-type rotations to the $\left(j_{3}, j_{4}\right)$ data qubits. Simultaneously, Igor uses the parity of $\left(M_{1}^{z}, M_{z}^{z}\right)$ to infer the eigenvalue information of the stabilizer. Once again, he proceeds with the readout of the remaining stabilizers in a modular fashion, which requires an intermediate reordering of the data qubits (i.e., bringing the ions belonging to the stabilizer to be measured into the storage zone $S_{2}$ ). Once this is achieved, the sequence of operations is again described by the realspace representation of Fig. 22.

To take full advantage of the fault-tolerant nature of the scheme, Igor must perform two or three rounds of stabilizer readout and then apply a minimum-weight decoder to determine which error has occurred and how to correct it.

\section{NUMERICAL STUDIES OF THE PERFORMANCE OF TRAPPED-ION QEC PROTOCOLS}

\section{A. Computing resources and numerical approach}

Having established our criterion for beneficial QEC in Eqs. (2) and (3), and described the different trapped-ion protocols together with their quantum-channel description in Figs. 15-22, let us now describe our numerical approach to assess the performance of these QEC schemes.

Our strategy for numerical analysis is to perform exact simulation of the physical system using pure states in a Monte Carlo method. The results of the simulation are achieved by averaging over the output of at least tens of thousands of individual runs; in each run, at each opportunity for an error event, the question of whether it occurs (and, when relevant, what the error's severity is) is resolved by drawing a random number. Once sufficiently many results are aggregated, one obtains the same data as would result from a single numerical run using a density matrix.
The advantages are twofold: First, the memory requirements of the pure state simulation are more modest, allowing for a simulation on the order of 30-40 qubits. A direct simulation of 30 qubits with the density matrix approach would require a matrix of $2^{60} \approx 10^{18}$ elements, which is infeasible. This will become crucial when considering fault-tolerant schemes and also when upgrading these protocols to include larger-distance codes or instances with more than one logical qubit. The second advantage of the Monte Carlo approach is that it is trivial to parallelize; thus, one can fully make use of cluster computing resources.

The hardware used for this work is a cluster of approximately 400 nodes, each of which is based on a motherboard with two Intel E5-2640v3 CPUs and between 64 and 256 GB of memory. The nodes are connected by Intel TruScale QDR Infiniband, and, in principle, they can be efficiently used to collectively model a quantum system. However, in practice, the efficient use is to operate the nodes in parallel and independently of one another, aggregating results afterwards according to the Monte Carlo paradigm outlined above.

\section{B. Simulation results}

In this subsection, we present the simulation results for all the different QEC protocols discussed above. One type of QEC cycle is based on the multiqubit MS gate (see Figs. 15 and 18), whereas the other is based on the sequential two-qubit MS gates, including both the nonfault-tolerant (see Fig. 19) and the fault-tolerant approaches (see Figs. 21 and 22). Generally, we will use the same Alice-Igor-Bob framework for assessment of the beneficial character of QEC, which has been discussed earlier: Alice prepares a perfect instance of the encoded qubit, and this logical qubit is then subjected to a period of environmental exposure during which Igor may perform one or more cycles of error correction before Bob assesses the integrity of the qubit by attempting to determine the encoded state (from one of the two choices). For the simulations with multiqubit MS gates, the initial encoded state was chosen randomly, while for those simulations with two-qubit MS gates, since they proved to show more encouraging results, we only let Alice encode the $|+\rangle$ state since it is the most vulnerable state under dephasing environmental noise. Therefore, Bob's success probability $\mathcal{P}_{B}$, shown in the following two sections, has two ranges: The first is from 1 to 0.75 , while the second varies from 1 to 0.5 ; however, both cases correspond to the complete decay of the qubit from full coherence to total decoherence.

For each of these cases, two sets of figures are presented depending on the choice of trapped-ion parameters: First, we present a set of figures drawn from the best fidelities that have been reported to date in relevant experiments. An exception is the single-species shuttling-based scheme and the two-species shuttling-based DVS scheme, where the 
TABLE V. MS-gate infidelities in different QEC cycle scenarios: We use our model of the MS gate $\varepsilon=\varepsilon_{m}+\varepsilon_{d}+\varepsilon_{I}$ in Eqs. (17), (19), and (20) to reproduce the current performance of five-ion MS gates in Table II. Then, we use the model to predict the infidelities for the stabilizer mappings for the scenarios of QEC with a warmer ion background and those with the expected improved conditions.

\begin{tabular}{lcccccc}
\hline \hline & \multicolumn{3}{c}{ Ion-crystal reconfiguration } & & \multirow{2}{*}{ Spectroscopic decoupling } \\
\cline { 2 - 4 } Approach & \multicolumn{2}{c}{ One species without recooling } & Two species with recooling & & \multirow{2}{*}{ Two species with recooling } \\
\hline Five-ion MS infidelity & Current value & Anticipated value & Current value & Anticipated value & Current value & Anticipated value \\
Map $S_{1}^{x}$ & $2.1 \%$ & $0.29 \%$ & $4.9 \%$ & $0.21 \%$ & $4.9 \%$ & $0.21 \%$ \\
Map $S_{1}^{z}$ & $5.2 \%$ & $0.46 \%$ & $4.9 \%$ & $0.21 \%$ & $4.9 \%$ & $0.21 \%$ \\
Map $S_{2}^{x}$ & $\ldots$ & $0.85 \%$ & $4.9 \%$ & $0.21 \%$ & $4.9 \%$ & $0.21 \%$ \\
Map $S_{2}^{z}$ & $\ldots$ & $1.5 \%$ & $4.9 \%$ & $0.21 \%$ & $4.9 \%$ & $0.21 \%$ \\
Map $S_{3}^{x}$ & $\ldots$ & $2.4 \%$ & $4.9 \%$ & $0.21 \%$ & $4.9 \%$ & $0.21 \%$ \\
Map $S_{3}^{z}$ & $\ldots$ & $3.2 \%$ & $4.9 \%$ & $0.21 \%$ & $4.9 \%$ & $0.21 \%$ \\
\hline \hline
\end{tabular}

performance of the MS gates after a few stabilizer mappings is expected to be so bad that one can directly discard the approach (see Tables II-V). Second, we present a set of figures for the future parameters that we anticipate will be possible to reach in the near future. We refer to these two cases as the "Current" and the "Future" (or "anticipated") performance, respectively.

\section{Shuttling-and hiding-based QEC with multiqubit MS gates}

Figures 23-27 constitute the results of our first set of simulations, i.e., those involving MS gates with multiple qubits (five qubits, specifically). Note that for these simulations, the qubit-reset fidelity is assumed to be $5 \times 10^{-3}$ (see Table II). The results shown in each figure correspond to one of the trapped-ion QEC protocols described in detail in the previous section, and we refer to them here as "single-species shuttling without cooling" (shuttling-based protocol 1, in Fig. 24), "dual-species shuttling with cooling" (shuttling-based protocol 2, in Figs. 25 and 26), and "hiding" (hiding-based protocol, in Figs. 23 and 27).

Each data point in our figures is the averaged result of at least 40000 runs. Each curve is formed from 200 data points and therefore involves eight million runs in total. For reference, the grey curves show how a single physical qubit would perform if used in the same setting described in Sec. II; i.e., Alice prepares it in a given state and Bob measures it to guess the state (vs the orthogonal state) after the state has been subjected to environmental noise. Moreover, the red curve in each figure shows the performance of the color code of seven physical qubits but without the error correction provided by Igor midway through the time $\tau$ during which the encoded state is subjected to environmental noise. The blue curves show how this changes when Igor's cycle is indeed performed. Regrading the criteria for beneficial QEC discussed in Sec. II, we wish to see the blue curve above the red one such that criterion [Eq. (2)] is achieved, and ideally even above the grey one, implying that (at least for some choices of duration of the experiment) Eq. (3) is also fulfilled. If our simulations display such crossing, we can conclude that advantageous QEC could be achieved in the experiments, given that the particular performance of the different

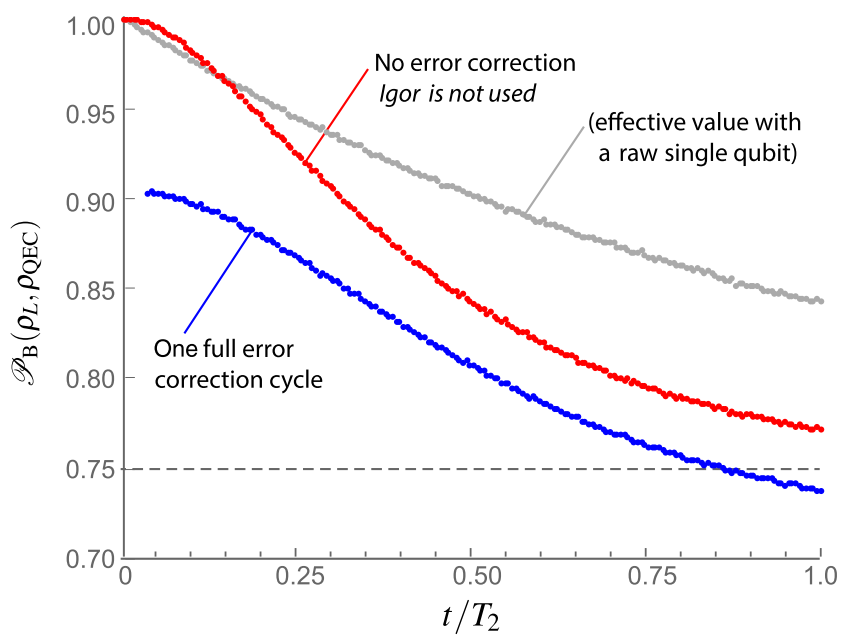

FIG. 23. Success probability $\mathcal{P}_{\mathrm{B}}$ under the hiding-based, twospecies protocol QEC cycle with multiqubit MS gates (cf. Fig. 20): The parameters underlying the simulation correspond to the current values from Tables II-V. Here and in the following figures, the $x$ axis gives time in units of the environmental dephasing time $T_{2}$. The noise model for the imperfect five-ion MS gate operations corresponds to the quantum channel (i) of independent depolarizing noise [Eq. (14)]. Results show that even for this optimistic noise model, there is no time window in which the application of an imperfect QEC cycle is beneficial as compared to not applying it [i.e., the regime in Eq. (2) is never attained]. The underlying reason for this is that the current fiveion MS gate fidelity is insufficient to reach the crossover point. Although not shown in the figure, we note that similar results are found for the shuttling-based protocol 2, for which reaching the crossover point with current parameters is not possible. Here and elsewhere, each random qubit selected for Alice to encode is $U|0\rangle$, where unitary $U$ is formed by selecting three angles $\phi_{1}, \phi_{2}$, and $\phi_{3}$ uniformly from 0 to $2 \pi$ and setting $U=\cos \phi_{1}\left(\cos \phi_{2} I+i \sin \phi_{2} Z\right)+i \sin \phi_{1}\left(\cos \phi_{3} Y+\sin \phi_{3} X\right)$, where $I$ is the identity and $X, Y, Z$ are the Pauli operators. 


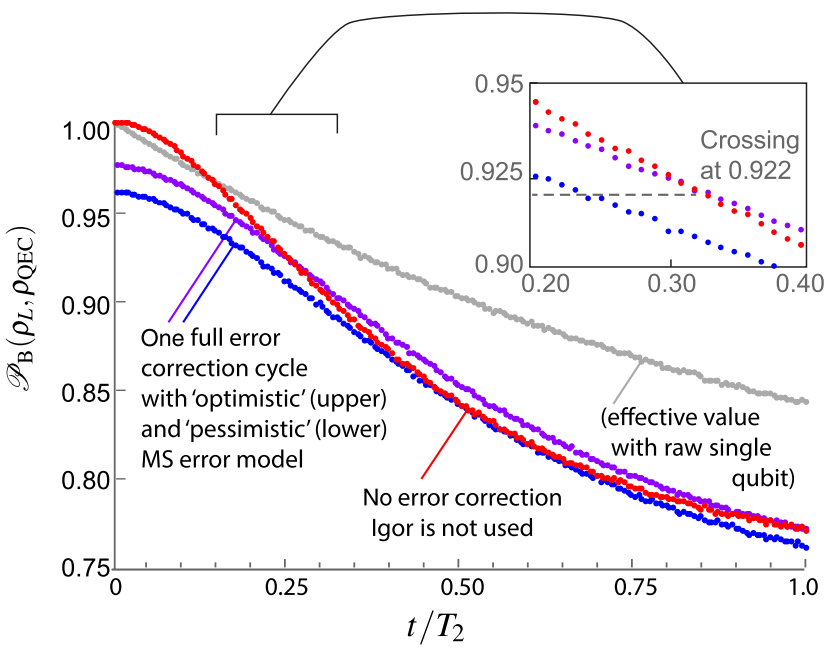

FIG. 24. Success probability $\mathcal{P}_{\mathrm{B}}$ under the single-species, shuttling-based QEC cycle with multiqubit MS gates (cf. Fig. 17): The parameters underlying the simulation correspond to the anticipated improved values from Tables II-V. We use two noise models for the imperfect five-ion MS gate operations involved in the stabilizer mappings: the optimistic model (i) of independent depolarizing channels [Eq. (14)] and the pessimistic model (iii) with a multiqubit depolarizing channel [Eq. (16)]. When we adopt the optimistic noise model and we employ Igor (purple curve), then for $t / T_{2}>0.3$ there exists a small advantage as compared to not using Igor to correct the logical qubit (red curve). Thus, Eq. (2) is fulfilled. However, when multiple qubit errors are fully enabled by the noise model, and we use the multiqubit depolarizing channel ("Igor," blue data points), the advantage disappears. This highlights the importance of modeling correlated errors appropriately, thus going beyond simplified error models that use the same single-qubit channel after each elementary operation in the quantum circuit. For reference, the behavior of an unencoded, bare physical qubit under the same environmental (dephasing) noise is also shown (grey data points). The inset shows a zoom of the parameter interval in which QEC becomes advantageous: For a total waiting time $\tau$ larger than about $300 \mathrm{~ms}$, it becomes advantageous to apply an imperfect Igor QEC cycle but only for independent depolarizing noise. In this figure and those following, the inset shares the same axes labels as the main plot.

building blocks is realized. We note that in Fig. 26 there is an additional curve, in green, which shows the effect of applying Igor's correction twice, at $33 \%$ and $67 \%$ of the Alice-Bob time interval; again, one hopes to see the curves associated with Igor surpass the red or even the grey curves.

From our numerical simulations (see Fig. 23 for the hiding approach), we can conclude that the "Current" performance figures for the gate times, fidelities, and so on, would be insufficient, in general, to prove a beneficial QEC cycle: The curves representing the integrity of the logical qubit at the end of the period $\tau$ are strictly lower when error correction is applied midway vs simply omitting any such correction. This is principally caused by the complexity of the required circuits and by the higher error rate of the entangling MS gates as compared to other building blocks of the protocol. Essentially, in these low-distance codes, the MS gates introduce more noise than can be removed by the QEC cycle. For the shuttlingbased approaches with current parameters (not shown in figures), a similar poor performance is found [e.g., for the one-species scheme without recooling, the MS gates become so noisy after a few rounds (see Table V) that we can directly discard it from reaching the breakeven point, Eq. (2)].

Remarkably, our results for the "Future" performance are far more encouraging. The least successful hardware variant is QEC protocol based on a single-species shuttling without recooling (cf. Fig. 24). For this protocol, the crossing into beneficial effects of the QEC cycle [Eq. (2)] occurs only when the total time $\tau$ is such that the logical qubit receives considerable dephasing from the environment. Moreover, the beneficial effect can vanish entirely when one moves to a more pessimistic model for the MS gate noise including correlations [noise models (ii) and (iii) discussed in Sec. IV B]. Additionally, the desirable property of outperforming the unprotected physical qubit in

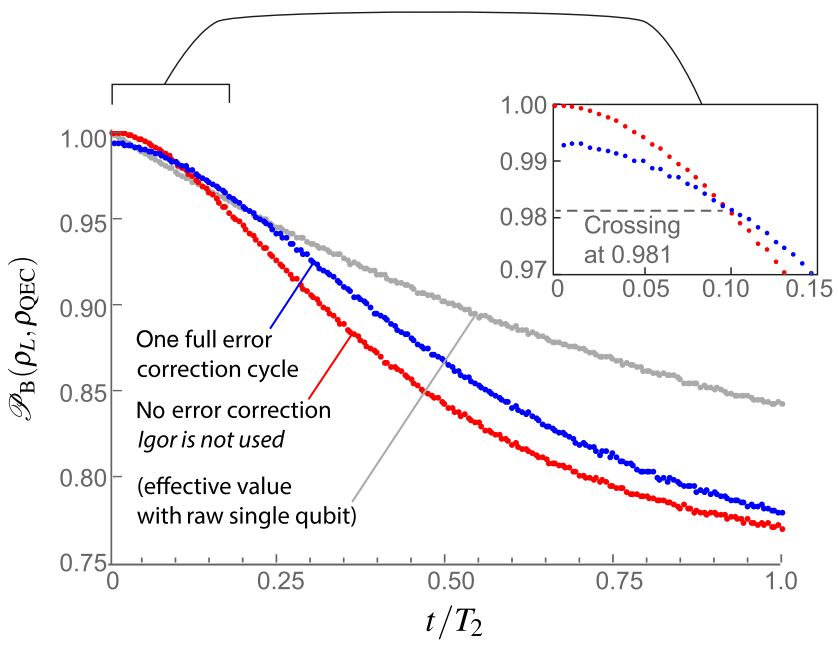

FIG. 25. Success probability $\mathcal{P}_{\mathrm{B}}$ under the two-species, shuttling-based QEC cycle with multiqubit MS gates (cf. Fig. 18): The parameters underlying the simulation correspond to the anticipated improved values from Tables II-V. The noise model for the imperfect five-ion MS gate operations corresponds to the worstcase noise model (iii) of multiqubit depolarizing noise [Eq. (16)]. Results show that there exists an ample parameter region (at times larger than about $100 \mathrm{~ms}$ ) in which the application of an imperfect Igor QEC cycle becomes advantageous [Eq. (2)] as compared to not applying it. Note that this takes place at $\mathcal{P}_{\mathrm{B}}$ values of 0.981 , much higher than in the shuttling-based scenario 1, with a marginal gain at $\mathcal{P}_{\mathrm{B}}$ values of about 0.92 . Note that in the present scheme, for not-too-long total times $\tau$, below about $200 \mathrm{~ms}$, applying an imperfect QEC cycle is advantageous even as compared to a single, nonencoded physical qubit undergoing dephasing noise of the same strength, such that the more-stringent regime [Eq. (3)] for beneficial QEC can also be achieved. 


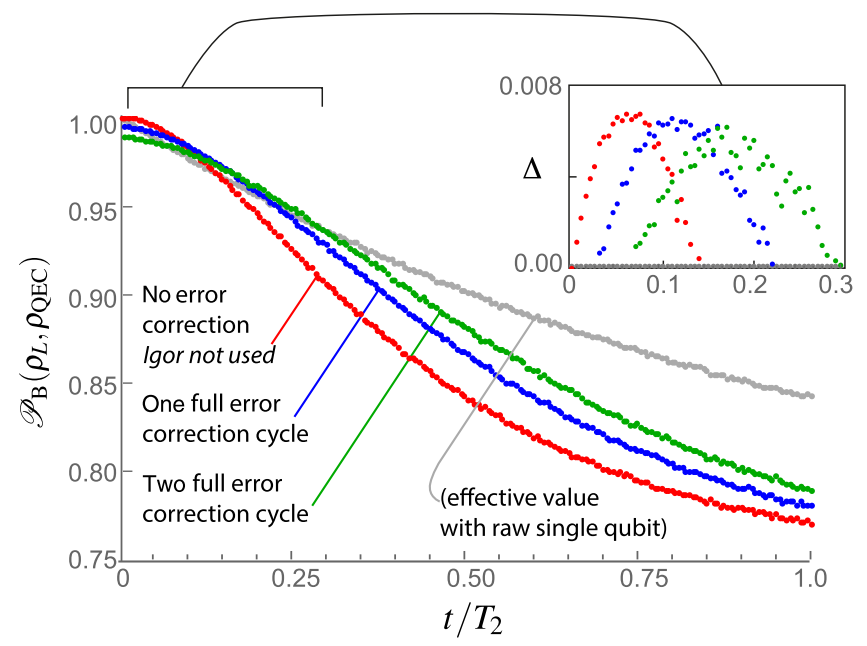

FIG. 26. Success probability $\mathcal{P}_{\mathrm{B}}$ under repetitive, two-species, shuttling-based QEC cycles with multiqubit MS gates (cf. Fig. 18): We consider the same scenario as in Fig. 25 with the anticipated improved values of Tables II-V, but the model for the imperfect five-ion MS gate operations corresponds to the physically motivated noise model (ii) of one- and two-qubit depolarizing quantum channels [Eq. (15)]. For a single QEC cycle, direct comparison to the results of Fig. 25 shows no appreciable difference. Hence, we can conclude that using the morepessimistic noise model with a multiqubit depolarizing channel [Eq. (16)] or using the one with equally likely one- and two-qubit errors [Eq. (15)] does not make any difference. We also depict the results for two rounds of QEC (green dots), where one sees an increase of the region [Eq. (3)] where QEC is advantageous compared to a single nonencoded physical qubit with respect to the case with a single QEC round. The inset plots $\Delta$, which is $\mathcal{P}_{\mathrm{B}}$ relative to that for the single-qubit memory. We see that multiple rounds of QEC allow us to sustain the logical qubit for a longer period of time. In the inset, we display the relative performance $\Delta$ of the encoded (red), single-cycle QEC (blue), and two-cycle QEC (green) with respect to the unprotected physical qubit, which is obtained by subtracting the bare qubit $\mathcal{P}_{\mathrm{B}}$ of the main panel (grey line), from $\mathcal{P}_{\mathrm{B}}$ for the different schemes, also in the main panel. As emphasized before, we observe a wider region of advantage for the two cycles of QEC.

our particular task of state discrimination through the QEC cycle [Eq. (3)] cannot be achieved. Fortunately, the results for the two-species shuttling (cf. Figs. 25 and 26) and the hiding protocols (cf. Fig. 27), using the future performance numbers, are considerably more encouraging. One sees that the crossing to a beneficial error-correction cycle happens early and with a high value of integrity. Importantly, this implies that multiple rounds of error correction can be beneficially applied such that the logical qubit can be sustained for a longer time (see Fig. 26).

Let us now address if one could obtain still better results by implementing the QEC using sequential two-qubit MS gates rather than multiqubit MS gates. While this would necessitate more gates in total, each gate has a higher fidelity and, moreover, would propagate errors from the ancillary qubits onto the data qubits in a more restricted

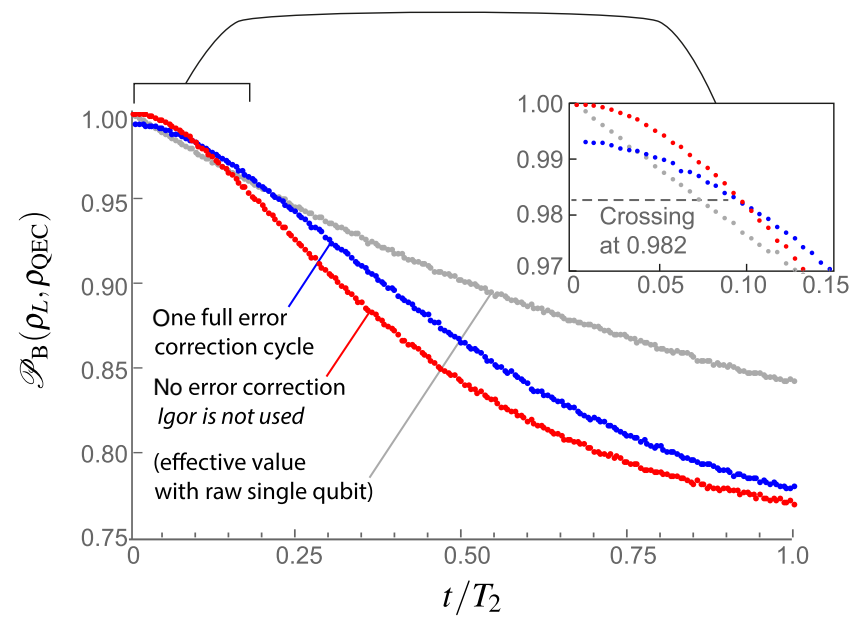

FIG. 27. Success probability $\mathcal{P}_{\mathrm{B}}$ under the hiding-based, twospecies QEC cycle with multiqubit MS gates (cf. Fig. 20): The parameters underlying the simulation correspond to the future improved values from Tables II-V. Experimental capabilities (two-species, cooling, etc.) are the same as in Fig. 23, and we use the more challenging (worst-case) noise model (iii) of multiqubit depolarizing noise [Eq. (16)]. Results show that there exists a clear parameter window for which the application of an imperfect Igor QEC cycle becomes advantageous both as compared to not applying it [Eq. (2)] and compared to an unprotected single physical qubit [Eq. (3)]. Note that the $\mathcal{P}_{\mathrm{B}}$ value where the QEC cycle crossover towards a beneficial Igor takes place is around 0.982 , which is very similar to the behavior found for the shuttling-based protocol 2 with future parameters (cf. Fig. 25).

fashion. In addition to addressing this question numerically, the sequential two-qubit MS gates will be an essential ingredient for the realization of fault-tolerant QEC, which we also explore in this section. The necessary analysis and scheduling for this second approach was described above in Sec. VI B. We now describe the results of the corresponding set of simulations, which employ the two-qubit MS gate as the entangling operation. For this second set of simulations, we also assumed the better value of $1 \times 10^{-3}$ for the qubit-reset fidelity from Table II.

\section{Shuttling-based, two-species QEC with two-qubit MS gates: Exploring fault tolerance}

Before presenting the simulation results to explore the breakeven point for the full fault-tolerant QEC protocols, let us first present a simpler analysis to verify that the DiVincenzo-Shor and DiVincenzo-Aliferis schemes with MS gates of Sec. V are indeed fault tolerant in the formal sense. To achieve this, the periods $\tau$ of environmental exposure in the Alice-Igor-Bob scenario are removed. Instead, Alice presents the flawless logical qubit directly to Igor, who performs a redundant round of error correction and then directly passes the logical qubit to Bob for the logical state discrimination. Hence, there is no effect of the environmental noise, except during the time when Igor is 
applying his imperfect error-correction cycle. We introduce a parameter $\lambda$ to control the severity of the imperfections in the operations applied by Igor, which multiplies the error rates that have been identified as the expected hardware targets (see Tables II-V). Setting $\lambda=1$ corresponds to assuming that all these targets are exactly met. By plotting Bob's success probability $\mathcal{P}_{\mathrm{B}}$ as a function of $\lambda$, we should see a linearly descending curve for a non-FT protocol (because any single gate failure within Igor's circuit can have the consequence of reducing $\mathcal{P}_{\mathrm{B}}$ ), whereas a characteristically inverted quadratic curve should arise for a true FT protocol (because it requires two or more gate failures to damage the integrity, the probability of which goes as $\lambda^{2}$ ). The results of our numerical simulations are shown in Fig. 28. The predicted shape of the curve is indeed observed through our numerics, thus verifying that the analytically derived protocols based on MS gates, and their translation into the numerical simulation, are correctly fault tolerant.

It is relevant to note that our approach of requiring betterthan-breakeven performance, when Igor enters the picture, is closely related to the concept of a pseudo-threshold where a higher level of concatenation outperforms the preceding level (see, e.g., Ref. [91]). Moreover, as we noted in the introduction to Sec. VI, thresholds in the range of $10^{-4}$ are expected in the context of fault tolerance [90,91]. Therefore, the crossing seen in Fig. 28 and the

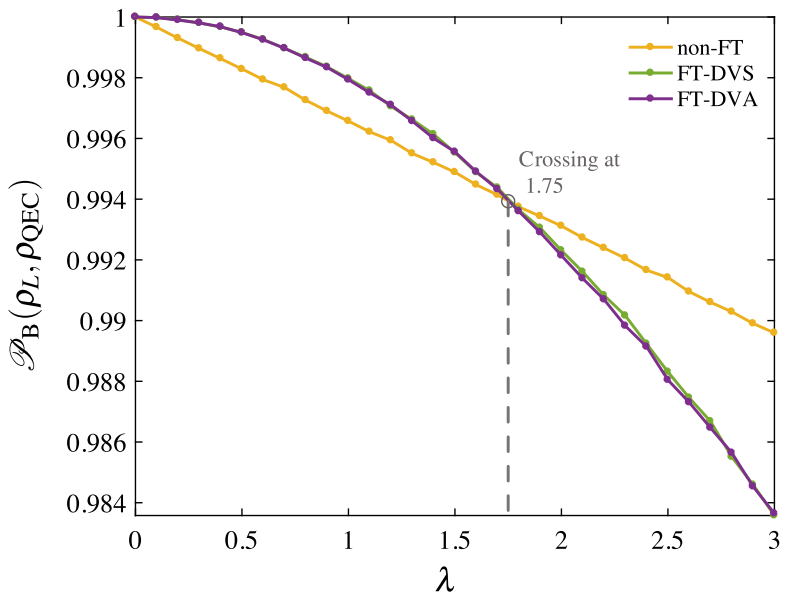

FIG. 28. Success probability $\mathcal{P}_{\mathrm{B}}$ under two-species, shuttlingbased QEC cycles with only QEC errors: This graph shows how $\mathcal{P}_{\mathrm{B}}\left(\rho_{L}, \rho_{\mathrm{QEC}}\right)$ changes with parameter $\lambda$, which is defined in the main text and adjusts error rates within Igor's cycle. The three plots show a non-fault-tolerant, two-species, shuttling-based scheme based on two-qubit MS gates (cf. Fig. 19), and the fault-tolerant DV-S scheme (cf. Fig. 21), and the fault-tolerant DV-A scheme (cf. Fig. 22). As described in the text, the inverted quadratic curves, as compared to the linear behavior, are the signature of a correct fault-tolerant circuit. The simulation parameters correspond to the future improved values from Tables II-V. The noise applied for the imperfect two-ion MS gate operations follows the standard depolarizing model. corresponding gate fidelities (i.e., 1.75 times those in Tables II-V) are reassuringly close to expectations. The very recent suggestions of Chao and Reichardt [92] for smaller ancilla structures in fault-tolerant circuits may provide some further boost to the transition.

Having thus verified the nature of the circuits, we can proceed to assess their performance when there are finite periods of exposure to the environment. First, we simulate using the current values of operational infidelities from Tables II-V. The results are shown in Fig. 29. In this figure, and the remaining figures in this section, the underlying protocol is that of two-species shuttling with recooling (as described in previous sections). Typically, for the figures in this section, each data point is aggregated from $10^{6}$ numerical experiments, and each full curve involves about 100 data points; thus, a curve represents approximately $10^{8}$ numerical experiments.

Figure 29 shows two reference lines for the criteria of beneficial QEC: The grey line indicates the performance with a single physical qubit [Eq. (3)], while the red line shows the performance with an encoded logical qubit when no error correction is performed by Igor [Eq. (2)]. As one would expect, for very short periods of environmental exposure, the red line lies above the grey one since the probability for two (or more) errors affecting the encoded data qubits is much smaller than the single-qubit error probability affecting the bare qubit. The yellow line shows

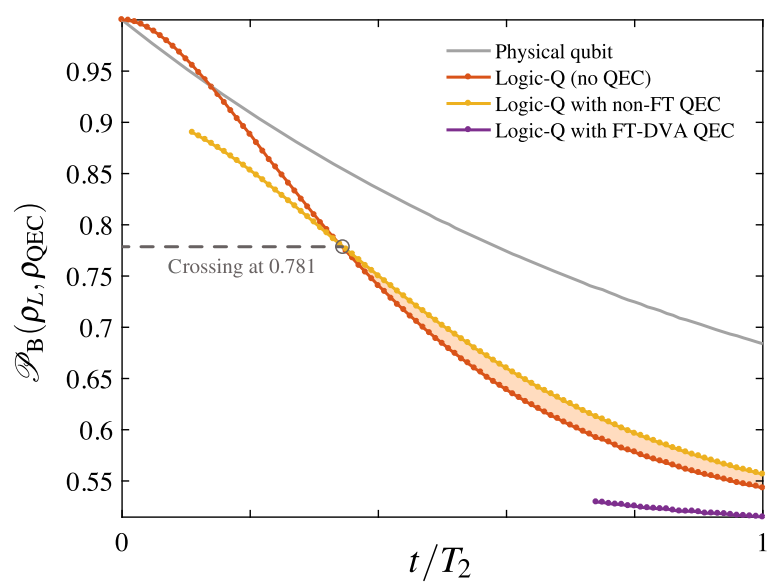

FIG. 29. Today's hardware: Success probability $\mathcal{P}_{\mathrm{B}}$ with QEC according to the shuttling-based, two-species protocol. The standard Alice-Igor-Bob protocol with the parameters underlying the simulation corresponding to the current values from Tables II-V. The red curve shown here is Bob's performance in the absence of Igor, such that the occurring errors only come form the environment (see Table I). For reference, the equivalent plot for a single physical qubit with the same environmental noise is also shown (grey curve). We see that using a logical qubit and the non-FT QEC cycle (yellow) can produce a small positive effect; the shaded area indicates this beneficial region. However, the fully FT protocol (purple line), when it is possible (see main text), is always inferior. 
TABLE VI. Resource overview for the different QEC protocols. We describe the number of various operations that conform each QEC cycle [i.e., measurement of all the six stabilizers, Eq. (23)] within the different trapped-ion protocols introduced in Sec. VI. The number of basic operations can be obtained from the different schemes presented in the Figs. 15-22. In the two rightmost columns, we present the total time required for each QEC cycle according to the current and anticipated values given in Tables II and IV. Note, however, that some of the local single-qubit gates require the use of refocusing techniques that combine various single-qubit gates and that some operations can be done in parallel in different manipulation zones of the ion trap. The latter can lead to a minimization of the overall time of the QEC cycle (see Table VII for the optimized QEC cycle times).

\begin{tabular}{|c|c|c|c|c|c|c|c|c|c|}
\hline & $\begin{array}{l}\text { Two-ion } \\
\text { MS gate }\end{array}$ & $\begin{array}{l}\text { Five-ion } \\
\text { MS gate }\end{array}$ & $\begin{array}{l}\text { Single-qubit } \\
\text { gate }\end{array}$ & Meas. & Recooling & $\begin{array}{l}\text { Split, shuttle, } \\
\text { and merge }\end{array}$ & Rotation & $\begin{array}{c}\text { Total time } \\
\text { (current) } \\
(\mathrm{ms})\end{array}$ & $\begin{array}{c}\text { Total time } \\
\text { (anticipated) } \\
\text { (ms) }\end{array}$ \\
\hline \multicolumn{10}{|l|}{$\begin{array}{l}\text { Non-fault-tolerant trapped-ion } \\
\text { QEC protocols }\end{array}$} \\
\hline $\begin{array}{l}\text { Shuttling-based, single-species } \\
\text { multiqubit gate (A.1.) }\end{array}$ & $\ldots$ & 12 & 42 & 6 & $\ldots$ & 20 & 2 & 6.7 & 1.7 \\
\hline $\begin{array}{l}\text { Shuttling-based, two-species } \\
\text { multiqubit gate (A.2.) }\end{array}$ & $\cdots$ & 12 & 42 & 6 & 6 & 6 & 2 & 6.8 & 1.4 \\
\hline $\begin{array}{l}\text { Shuttling-based, two-species } \\
\text { two-qubit gate (A.3.) }\end{array}$ & 24 & $\ldots$ & 48 & 6 & 24 & 54 & 36 & 23.6 & 7.2 \\
\hline $\begin{array}{l}\text { Hiding-based, two-species } \\
\text { multiqubit gate (A.4.) }\end{array}$ & $\ldots$ & 12 & 150 & 6 & 6 & $\ldots$ & $\cdots$ & 6.3 & 1.1 \\
\hline \multicolumn{10}{|l|}{$\begin{array}{l}\text { Fault-tolerant trapped-ion } \\
\text { QEC protocols }\end{array}$} \\
\hline $\begin{array}{l}\text { Shuttling-based, two-species } \\
\text { DiVincenzo-Shor (B.1.) }\end{array}$ & 54 & $\ldots$ & 84 & 24 & 54 & 190 & 150 & 71.2 & 22.4 \\
\hline $\begin{array}{l}\text { Shuttling-based, two-species } \\
\text { DiVincenzo-Aliferis (B.2.) }\end{array}$ & 54 & $\cdots$ & 78 & 24 & 54 & 190 & 144 & 71.0 & 22.2 \\
\hline
\end{tabular}

the performance of the non-FT QEC protocol that employs sequential two-qubit MS gates (cf. Fig. 19). As can be observed, while this line never beats the $\mathcal{P}_{\mathrm{B}}$ for the bare physical qubit [Eq. (3)], it does indeed exceed the performance of the encoded qubit [Eq. (2)] when the environmental exposure is severe. Note that this result indicates the superior performance of the QEC schemes with two-ion MS gates vs the previous ones based on multi-ion MS gates. We recall that for the corresponding multi-ion MS circuits, no clear advantage could be seen using current hardware performance; i.e., there was no equivalent to the yellowred crossing of Fig. 29. Let us remark that this result is nontrivial since the complexity of the circuits using sequential two-ion MS gates increases considerably with respect to the schemes that exploit multiqubit MS gates (compare Fig. 19 to Fig. 18). In any case, the breakeven point [Eq. (2)] is achieved when the integrity of the qubit has already decayed considerably. In order to take full advantage of QEC, improving on this feature, we now consider the DV-S and DV-A schemes for fault-tolerant QEC.

The purple line of Fig. 29, just visible in the lower-right corner of the plot, is the performance of the DV-A protocol. The line does not exist over the majority of the graph simply because there is insufficient time to perform a full error-correction cycle because of the long circuit depth associated with Fig. 22. The time required for Igor's actions is summarized in Tables VI and VII. Note that for the FT approaches, it is necessary to evaluate each stabilizer more than once in order to control measurement errors; Igor performs the checks twice and then a third time if the first two outcomes do not agree. For the Shor FT scheme, it is necessary to prepare and verify an ancilla state prior to stabilizer measurement. If the verification fails, we must restart the ancilla preparation. In our simulations, we allow for up to four such restarts; the probability that more are needed is negligible even for current technologies.

Notice that in Fig. 29, even when the total protocol time is large enough to permit Igor to act, the performance of the DV-A scheme is very poor, and none of the breakeven points for useful QEC can be reached. The conclusion from

TABLE VII. Time required for one cycle of error correction with both current and future hardware, assuming the shuttlingbased, two-species protocol. Parameter $n$ is the number of rounds of error correction applied (usually $n=2$, but $n=3$ when the initial two rounds disagree). Parameter $m$ is the number of additional attempts at preparing the GHZ ancilla state beyond the minimum because of detection of error(s) when verifying the ancilla. On average, $m=0.66$ for current hardware and $m=0.02$ for future hardware.

\begin{tabular}{lcc}
\hline \hline Protocol & $\begin{array}{c}\text { Total time } \\
\text { (current) }(\mathrm{ms})\end{array}$ & $\begin{array}{c}\text { Total time } \\
\text { (anticipated) }(\mathrm{ms})\end{array}$ \\
\hline non-FT & 21.2 & 5.9 \\
FT DVA & $49.3 \times n$ & $14.3 \times n$ \\
FT DVS & $46.0 \times n+3.7 \times m$ & $13.1 \times n+1.0 \times m$ \\
\hline \hline
\end{tabular}




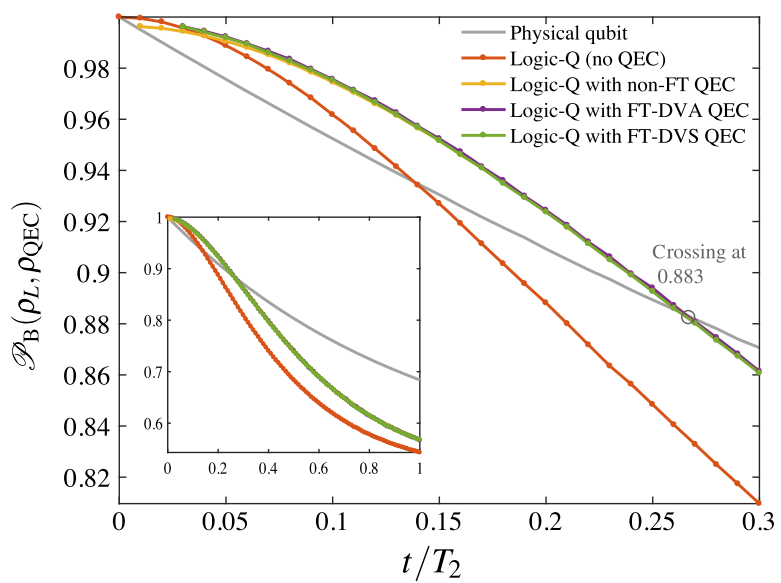

FIG. 30. Future hardware: Success probability $\mathcal{P}_{\mathrm{B}}$ with QEC according to the shuttling-based, two-species protocol. The scenario simulated in this case is the same as in Fig. 29, except that the parameters applied correspond to the future improved values from Tables II-V. Performance is obviously profoundly improved, as discussed in the main text.

this set of simulations is that a device built with the "current" performance numbers could suffice for a basic demonstration of QEC but could not possibly make a successful demonstration of a fault-tolerant QEC code. Fortunately, this picture changes as we move to the future performance figures.

Figure 30 shows the results of repeating the simulations in Fig. 29 but now with the future anticipated values from Tables II-V. One can see that the performance is profoundly improved. Now, the lines for the non-FT Igor, and both the DV-A and DV-S fault-tolerant protocols, lie almost on top of one another. Moreover, they both beat the "no-Igor" red line, fulfilling Eq. (2) for the whole period of time studied numerically. Additionally, they also lie above the "single physical qubit" grey line, fulfilling Eq. (3), over a wide range of values of the environmental exposure time. Finally, we note that for longer times, one could use multiple rounds of error correction. Although the non-FT curve and the two FT curves seem nearly identical, the latter outperform the non-FT protocol for small levels of environmental error (see top left of the figure). This is consistent with our expectations from Fig. 28, where we learned that, when Igor's hardware performs exactly at the future anticipated level (i.e., $\lambda=1$ ), the FT protocols are superior to the non-FT one.

The curves in Fig. 30 are so encouraging that the trapped-ion hardware development may not need to reach the expected values of Tables II-V in order to achieve the goal of beneficial QEC. In order to test this feature, we have tripled the error rates in all operations and analyzed the performance for the QEC procedures. The results are shown in Fig. 31. We see that now there is a slight variation in performance with the non-FT Igor marginally superior to FT-DV-A, which in turn is marginally superior to FT-DV-S.

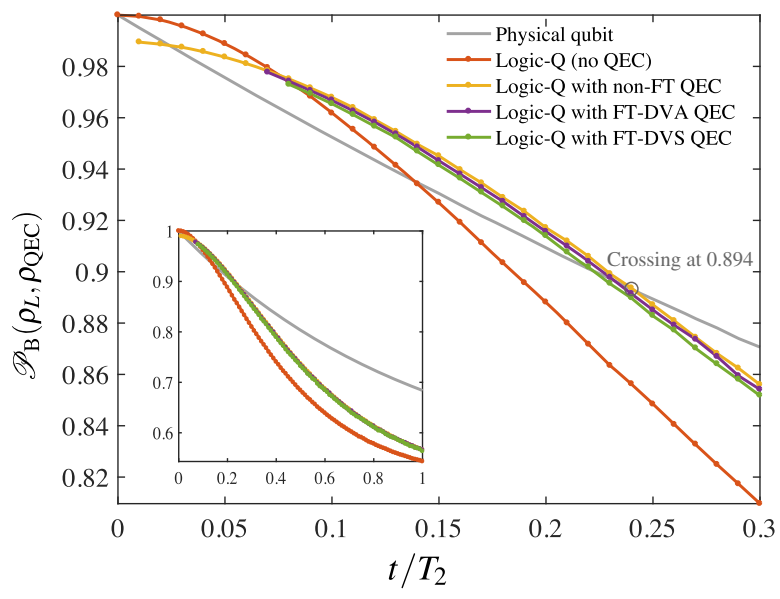

FIG. 31. Inferior future hardware: Success probability $\mathcal{P}_{\mathrm{B}}$ with QEC according to the shuttling-based, two-species protocol. The error model and scenarios simulated here are the same as in Fig. 30. The operational numbers are scaled to yield a performance that is 3 times worse than those of the future improved values: Each operation takes 3 times longer, and the gate fidelities are 3 times worse (the environmental dephasing rate remains unchanged). As discussed in the main text, while the performance here is degraded vs the preceding figure, it is nevertheless sufficient to largely demonstrate the goal of beneficial QEC.

(Note that it is to be expected that the FT circuits are now inferior to the non-FT circuit since by tripling the error we are now at the far right of the range in Fig. 28.) In Fig. 31, we also see that the non-FT Igor is quicker to perform than DV-A, which in turn is quicker than DV-S, since the curves are not plotted when there is an insufficient Alice-Bob time interval for a complete Igor cycle. Additionally the figure shows that the crossing of the curves with error correction and the physical qubit occurs only slightly earlier in Fig. 31 (0.894) than in Fig. 30. The conclusion from this figure is that, even using a system with 3 times greater operational infidelities with respect to the expected future estimates of our work, one could nevertheless support a strong demonstration of beneficial QEC. This is very encouraging for the near-term development of trapped-ion QEC.

In our final set of simulations, shown in Fig. 32, we consider multiple rounds of error correction considering the future expected levels of infidelity. These results show that, by several rounds of QEC, one can protect the logical qubit at a level that is superior to a single physical qubit over a sustained period of time. By the simple principle of selecting the number of Igor cycles according to the total Alice-Bob interval, we find that the coherence time of the logical qubit can reach values nearly twice as big as the raw physical qubit - a very significant alteration that should be easily observed and that demonstrates the "encoded qubit alive" goal clearly. Of course, a factor of 2 is far from sufficient to achieve large-scale quantum computing, but this is to be expected since we are employing only one layer of a small code. For further error suppression, one would 


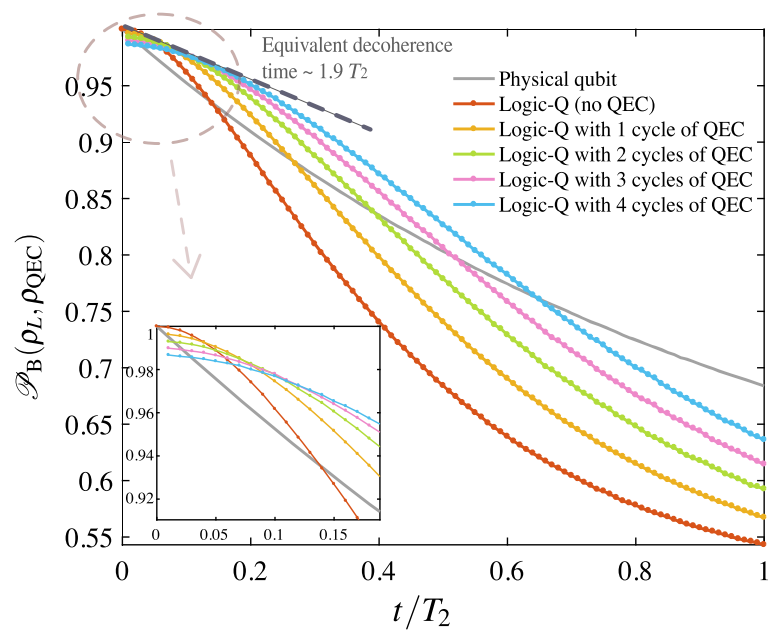

FIG. 32. Multiple cycles of future hardware: Success probability $\mathcal{P}_{\mathrm{B}}$ with QEC according to the shuttling-based, two-species protocol. The parameters underlying the simulation correspond to the future improved values from Tables II-V. The QEC method used here is the non-fault-tolerant one (cf. Fig. 19), as it takes the shortest possible time and thus has more potential if more errorcorrection cycles are to be applied within one round. Results show that the application of more QEC cycles sustains the logical qubit for a longer time, as depicted by the grey dashed line, which is drawn based on the envelope of the different curves. As discussed in the main text, the dashed line allows us to infer the effective rate of decay of integrity of the logical qubit.

either concatenate the Steane code (recursively replacing each data qubit with a full logical qubit, through at least three or four levels) or one would scale using topological techniques. As the Steane code is the smallest instance of the 2D color code, the latter would be an attractive optionbut such considerations are beyond the scope of the present paper.

This concludes our review of our numerical simulations. It is worth reiterating that, in every case, we presume that Alice and Bob are perfect since we are interested in the integrity of the memory itself, separate from the creation or analysis of the logical qubit. One may wonder whether this presents difficulties for experimental tests since, in reality, Alice, Igor, and Bob are merely phases of a single experiment and presumably suffer the same error rates. An analysis of this point is beyond the scope of the present paper, but it has been considered in a subsequent work [93]. The encouraging conclusion is that, for a broad class of error models (including the typical ones), experimental evaluation of integrity is possible even with noisy Alice and Bob. The key observation is that it typically suffices for Alice to randomly choose between a fixed subset of possible states to send; then, if one can find a fault-tolerant circuit for Alice to use to prepare each such state, and a corresponding fault-tolerant analysis method for Bob, one finds that the noisy nature of their actions has relatively little impact on the measured integrity.
Finally, we remark that while the error models considered here have been stochastic, the framework we have introduced applies to any form of noise; therefore, a full exploration of coherent and even non-Markovian noise is an interesting prospect. A recent paper has highlighted the potential for coherent errors to impact QEC performance in a qualitatively different way [94].

\section{CONCLUSIONS AND OUTLOOK}

In this work, we have presented a detailed description of current and future experimental capabilities for the implementation of topological QEC with trapped-ion crystals. We have also described the characteristics of the main sources of noise and imperfections in the experiments. Based on this discussion, we have introduced a complete trapped-ion toolbox for QEC, including a discussion of fault-tolerant designs based on the characteristics of the trapped-ion set of available gate operations. Using this toolbox, we have presented different protocols to implement a QEC cycle based on the seven-qubit color code, which exploit either crystal reconfiguration or spectroscopic decoupling or recoupling techniques. We have derived effective error models for the different building blocks of these QEC cycles, which are closely connected with the experimental sources of noise and go well beyond the simplified standard approaches that use quantum channels affecting all QEC operations with the same probability. Using these effective models for the current and expected performance of the QEC building blocks, we have performed extensive numerical simulations to determine the experimental conditions that are required for these QEC protocols to become beneficial, a fundamental and necessary condition for any future implementation of QEC. Moreover, we have also assessed the conditions for the encoded logical qubit to outperform the physical unprotected qubit for a particular quantum-information task.

From this study, and in light of our numerical results, we can draw the following conclusions. The performance of the seven-qubit trapped-ion color code for the single- or two-species, shuttling- or hiding-based, QEC protocols with multiqubit MS gates, and assuming the "current" performance of the experimental building blocks, is inadequate to achieve the breakeven point of beneficial QEC. Therefore, the trapped-ion hardware must be improved. Using the operational criteria [Eqs. (2) and (3)] introduced in this work, we have been able to assess and quantify the required experimental improvements towards the QEC goal and to present realistic values of the different building blocks that must be achieved (see Tables II-V). Our numerical results for the "future" expected improvements show that the crossing onto a beneficial QEC cycle can indeed be achieved with either the shuttling- or the hidingbased protocols with multiqubit MS gates and that it occurs at much earlier times and with a much higher value of the integrity of the encoded qubit. Therefore, we conclude that 
it will be of primary importance to incorporate and optimize the QEC building blocks towards the values introduced in Tables II-IV for the success of trapped-ion implementations of the QEC color code with two-species ion crystals. Moreover, we have observed a clear advantage of the QEC schemes based on sequential two-qubit MS gates, especially in the context of the fault-tolerant designs. Therefore, our studies show that the natural next step in the progress towards trapped-ion fault-tolerant QEC will be to upgrade the syndrome extraction routines according to the schemes introduced here. We finally note that, for the protocols presented, not only is the necessary condition for a beneficial QEC cycle [Eq. (2)] fulfilled, but we have also shown that the encoded qubit can perform better than the unprotected qubit [Eq. (3)]. Moreover, we have shown that repetitive QEC cycles can sustain the integrity of the logical qubit for increasing periods of time, provided that the above breakeven point is achieved.

\section{ACKNOWLEDGMENTS}

The research is based upon work supported by the Office of the Director of National Intelligence (ODNI), Intelligence Advanced Research Projects Activity (IARPA), via the U.S. Army Research Office Grant No. W911NF-16-1-0070. The views and conclusions contained herein are those of the authors and should not be interpreted as necessarily representing the official policies or endorsements, either expressed or implied, of the ODNI, IARPA, or the U.S. Government. The U.S. Government is authorized to reproduce and distribute reprints for Governmental purposes notwithstanding any copyright annotation thereon. Any opinions, findings, and conclusions or recommendations expressed in this material are those of the author(s) and do not necessarily reflect the view of the U.S. Army Research Office (ARO). We also acknowledge support by U.S. ARO through Grant No. W911NF-14-1-010. A. B. acknowledges support from Spanish MINECO Project No. FIS2015-70856-P, and CAM Regional Research Consortium QUITEMAD+. P. S., T. M., and R. B. acknowledge support from the Austrian Science Fund (FWF), through the SFB FoQus (FWF Project No. F4002-N16) and the Institut für Quanteninformation GmbH. S. C. B. acknowledges support from EPSRC Grant No. EP/M013243/1.

[1] M. A. Nielsen and I. L. Chuang, Quantum Computation and Quantum Information (Cambridge University Press, Cambridge, England, 2000).

[2] T. D. Ladd, F. Jelezko, R. Laflamme, Y. Nakamura, C. Monroe, and J. L. O'Brien, Quantum Computers, Nature (London) 464, 45 (2010).

[3] P. W. Shor, Scheme for Reducing Decoherence in Quantum Computer Memory, Phys. Rev. A 52, R2493 (1995).
[4] A. R. Calderbank and P. W. Shor, Good Quantum ErrorCorrecting Codes Exist, Phys. Rev. A 54, 1098 (1996).

[5] A. M. Steane, Error Correcting Codes in Quantum Theory, Phys. Rev. Lett. 77, 793 (1996).

[6] D. Aharonov and M. Ben-Or, Fault-Tolerant Quantum Computation with Constant Error, Proceedings of the 29th Annual ACM Symposium on Theory of Computation (ACM, New York, 1998); SIAM J. Comput. 38, 1207 (2008).

[7] A. Y. Kitaev, Fault-Tolerant Quantum Computation by Anyons, Ann. Phys. (Amsterdam) 303, 2 (2003).

[8] H. Bombin and M. A. Martin-Delgado, Topological Quantum Distillation, Phys. Rev. Lett. 97, 180501 (2006).

[9] D. G. Cory, M. D. Price, W. Maas, E. Knill, R. Laflamme, W. H. Zurek, T. F. Havel, and S. S. Somaroo, Experimental Quantum Error Correction, Phys. Rev. Lett. 81, 2152 (1998).

[10] E. Knill, R. Laflamme, R. Martinez, and C. Negrevergne, Benchmarking Quantum Computers: The Five-Qubit Error Correcting Code, Phys. Rev. Lett. 86, 5811 (2001).

[11] J. Chiaverini, D. Leibfried, T. Schaetz, M. D. Barrett, R. B. Blakestad, J. Britton, W. M. Itano, J. D. Jost, E. Knill, C. Langer, R. Ozeri, and D. J. Wineland, Realization of Quantum Error Correction, Nature (London) 432, 602 (2004).

[12] M. D. Reed, L. DiCarlo, S. E. Nigg, L. Sun, L. Frunzio, S. M. Girvin, and R. J. Schoelkopf, Realization of ThreeQubit Quantum Error Correction with Superconducting Circuits, Nature (London) 482, 382 (2012); D. Riste, S. Poletto, M.-Z. Huang, A. Bruno, V. Vesterinen, O.-P. Saira, and L. DiCarlo, Detecting Bit-Flip Errors in a Logical Qubit Using Stabilizer Measurements, Nat. Commun. 6, 6983 (2015).

[13] P. Schindler, J. T. Barreiro, T. Monz, V. Nebendahl, D. Nigg, M. Chwalla, M. Hennrich, and R. Blatt, Experimental Repetitive Quantum Error Correction, Science 332, 1059 (2011).

[14] N. M. Linke, M. Gutierrez, K. A. Landsman, C. Figgatt, S. Debnath, K. R. Brown, and C. Monroe, Fault-Tolerant Quantum Error Detection, arXiv:1611.06946.

[15] D. Nigg, M. Müller, E. A. Martinez, P. Schindler, M. Hennrich, T. Monz, M. A. Martin-Delgado, and R. Blatt, Quantum Computations on a Topologically Encoded Qubit, Science 345, 302 (2014).

[16] J. Kelly, R. Barends, A. G. Fowler, A. Megrant, E. Jeffrey, T. C. White, D. Sank, J. Y. Mutus, B. Campbell, Yu Chen, Z. Chen, B. Chiaro, A. Dunsworth, I.-C. Hoi, C. Neill, P. J. J. O'Malley, C. Quintana, P. Roushan, A. Vainsencher, J. Wenner, A. N. Cleland, and J. M. Martinis, State Preservation by Repetitive Error Detection in a Superconducting Quantum Circuit, Nature (London) 519, 66 (2015).

[17] A. D. Córcoles, E. Magesan, S. J. Srinivasan, A. W. Cross, M. Steffen, J. M. Gambetta, and J. M. Chow, Demonstration of a Quantum Error Detection Code Using a Square Lattice of Four Superconducting Qubits, Nat. Commun. 6, 6979 (2015).

[18] J. Cramer, N. Kalb, M. A. Rol, B. Hensen, M. S. Blok, M. Markham, D. J. Twitchen, R. Hanson, and T. H. Taminiau, Repeated Quantum Error Correction on a Continuously Encoded Qubit by Real-Time Feedback, Nat. Commun. 7, 11526 (2016). 
[19] M. Müller, A. Rivas, E. A. Martinez, D. Nigg, P. Schindler, T. Monz, R. Blatt, and M. A. Martin-Delgado, Iterative Phase Optimization of Elementary Quantum Error Correcting Codes, Phys. Rev. X 6, 031030 (2016).

[20] P. Schindler, D. Nigg, T. Monz, J. T. Barreiro, E. Martinez, S. X. Wang, S. Quint, M. F. Brandl, V. Nebendahl, and C.F. Roos, A Quantum Information Processor with Trapped Ions, New J. Phys. 15, 123012 (2013).

[21] P. Maunz, Sandia National Laboratories Report No. SAND2016-0796R 618951 (2016).

[22] A. Sørensen and K. Mølmer, Entanglement and Quantum Computation with Ions in Thermal Motion, Phys. Rev. A 62 , 022311 (2000).

[23] K. Mølmer and A. Sørensen, Multiparticle Entanglement of Hot Trapped Ions, Phys. Rev. Lett. 82, 1835 (1999).

[24] M. Müller, K. Hammerer, Y. Zhou, C. F. Roos, and P. Zoller, Simulating Open Quantum Systems: From Many-Body Interactions to Stabilizer Pumping, New J. Phys. 13, 085007 (2011).

[25] D. P. DiVincenzo and P. W. Shor, Fault-Tolerant Error Correction with Efficient Quantum Codes, Phys. Rev. Lett. 77, 3260 (1996).

[26] D. P. DiVincenzo and P. Aliferis, Effective Fault-Tolerant Quantum Computation with Slow Measurements, Phys. Rev. Lett. 98, 020501 (2007).

[27] D. Gottesman, Quantum Fault Tolerance in Small Experiments, arXiv:1610.03507.

[28] D. Kielpinski, V. Meyer, M. A. Rowe, C. A. Sackett, W. M. Itano, C. Monroe, and D. J. Wineland, A Decoherence-Free Quantum Memory Using Trapped Ions, Science 291, 1013 (2001); T. Monz, K. Kim, A. S. Villar, P. Schindler, M. Chwalla, M. Riebe, C. F. Roos, H. Häffner, W. Hänsel, M. Hennrich, and R. Blatt, Realization of Universal Ion-Trap Quantum Computation with Decoherence-Free Qubits, Phys. Rev. Lett. 103, 200503 (2009).

[29] T. Ruster, C. T. Schmiegelow, H. Kaufmann, C. Warschburger, F. Schmidt-Kaler, and U. G. Poschinger, A Long-Lived Zeeman Trapped-Ion Qubit, Appl. Phys. B 122, 254 (2016).

[30] C. J. Ballance, T. P. Harty, N. M. Linke, M. A. Sepiol, and D. M. Lucas, High-Fidelity Quantum Logic Gates Using Trapped-Ion Hyperfine Qubits, Phys. Rev. Lett. 117, 060504 (2016).

[31] J. P. Gaebler, T. R. Tan, Y. Lin, Y. Wan, R. Bowler, A. C. Keith, S. Glancy, K. Coakley, E. Knill, D. Leibfried, and D. J. Wineland, High-Fidelity Universal Gate Set for ${ }^{9} \mathrm{Be}^{+}$ Ion Qubits, Phys. Rev. Lett. 117, 060505 (2016).

[32] E. A. Martinez, C. A. Muschik, P. Schindler, D. Nigg, A. Erhard, M. Heyl, P. Hauke, M. Dalmonte, T. Monz, P. Zoller, and R. Blatt, Real-Time Dynamics of Lattice Gauge Theories with a Few-Qubit Quantum Computer, Nature (London) 534, 516 (2016).

[33] T. Monz, P. Schindler, J. T. Barreiro, M. Chwalla, D. Nigg, W. A. Coish, M. Harlander, W. Hänsel, M. Hennrich, and R. Blatt, 14-Qubit Entanglement: Creation and Coherence, Phys. Rev. Lett. 106, 130506 (2011).

[34] T. R. Tan, J. P. Gaebler, Y. Lin, Y. Wan, R. Bowler, D. Leibfried, and D. J. Wineland, Multi-element Logic Gates for Trapped-Ion Qubits, Nature (London) 528, 380 (2015).
[35] C. J. Ballance, V. M. Schäfer, J. P. Home, D. J. Szwer, S. C. Webster, D. T. C. Allcock, N. M. Linke, T. P. Harty, D. P. L. Aude Craik, D. N. Stacey, A. M. Steane, and D. M. Lucas, Hybrid Quantum Logic and a Test of Bell's Inequality Using Two Different Atomic Isotopes, Nature (London) 528, 384 (2015).

[36] H. Ball, W. D. Oliver, and M. J. Biercuk, The Role of Master Clock Stability in Quantum Information Processing, New Phys. J. Quantum. Info. 2, 16033 (2016).

[37] L. Viola and S. Lloyd, Dynamical Suppression of Decoherence in Two-State Quantum Systems, Phys. Rev. A 58, 2733 (1998).

[38] L. Viola, E. Knill, and S. Lloyd, Dynamical Decoupling of Open Quantum Systems, Phys. Rev. Lett. 82, 2417 (1999).

[39] P. Zanardi, Symmetrizing Evolutions, Phys. Lett. A 258, 77 (1999).

[40] D. Vitali and P. Tombesi, Using Parity Kicks for Decoherence Control, Phys. Rev. A 59, 4178 (1999).

[41] L. Viola and E. Knill, Robust Dynamical Decoupling of Quantum Systems with Bounded Controls, Phys. Rev. Lett. 90, 037901 (2003).

[42] M. S. Byrd and D. A. Lidar, Empirical Determination of Dynamical Decoupling Operations, Phys. Rev. A 67, 012324 (2003).

[43] K. Khodjasteh and D. A. Lidar, Fault-Tolerant Quantum Dynamical Decoupling, Phys. Rev. Lett. 95, 180501 (2005).

[44] W. Yao, R. B. Liu, and L. J. Sham, Restoring Coherence Lost to a Slow Interacting Mesoscopic Spin Bath, Phys. Rev. Lett. 98, 077602 (2007).

[45] G. S. Uhrig, Keeping a Quantum Bit Alive by Optimized $\pi$ Pulse Sequences, Phys. Rev. Lett. 98, 100504 (2007).

[46] G. Gordon, G. Kurizki, and D. A. Lidar, Optimal Dynamical Decoherence Control of a Qubit, Phys. Rev. Lett. 101, 010403 (2008).

[47] K. Khodjasteh and L. Viola, Dynamically Error-Corrected Gates for Universal Quantum Computation, Phys. Rev. Lett. 102, 080501 (2009).

[48] K. Khodjasteh and L. Viola, Dynamical Quantum Error Correction of Unitary Operations with Bounded Controls, Phys. Rev. A 80, 032314 (2009).

[49] K. Khodjasteh, D. A. Lidar, and L. Viola, Dynamical Quantum Error Correction of Unitary Operations with Bounded Controls, Phys. Rev. Lett. 104, 090501 (2010).

[50] W. Yang, Z. Y. Wang, and R. B. Liu, Preserving Qubit Coherence by Dynamical Decoupling, Front. Phys. China 6, 2 (2011).

[51] M. J. Biercuk, A.C. Doherty, and H. Uys, Dynamical Decoupling Sequence Construction as a Filter-Design Problem, J. Phys. B 44, 154002 (2011).

[52] Y. Wang, M. Um, J. Zhang, S. An, M. Lyu, J.-N. Zhang, L.-M. Duan, D. Yum, and K. Kim, Single-Qubit Quantum Memory Exceeding 10-Minute Coherence Time, arXiv: 1701.04195.

[53] H. K. Ng, D. A. Lidar, and J. Preskill, Combining Dynamical Decoupling with Fault-Tolerant Quantum Computation, Phys. Rev. A 84, 012305 (2011).

[54] N. C. Jones, R. Van Meter, A. G. Fowler, P. L. McMahon, J. Kim, T. D. Ladd, and Y. Yamamoto, Layered Architecture for Quantum Computing, Phys. Rev. X 2, 031007 (2012). 
[55] K. R. Brown, A. W. Harrow, and I. L. Chuang, Arbitrarily Accurate Composite Pulse Sequences, Phys. Rev. A 70, 052318 (2004).

[56] S. Wimperis, Broadband, Narrowband, and Passband Composite Pulses for Use in Advanced NMR Experiments, J. Magn. Reson. 109, 221 (1994).

[57] H. K. Cummins, G. Llewellyn, and J. A. Jones, Tackling Systematic Errors in Quantum Logic Gates with Composite Rotations, Phys. Rev. A 67, 042308 (2003).

[58] M. Bando, T. Ichikawa, Y. Kondo, and M. Nakahara, Concatenated Composite Pulses Compensating Simultaneous Systematic Errors, J. Phys. Soc. Jpn. 82, 014004 (2013).

[59] C. Kabytayev, T. J. Green, K. Khodjasteh, M. J. Biercuk, L. Viola, and K. R. Brown, Robustness of Composite Pulses to Time-Dependent Control Noise, Phys. Rev. A 90, 012316 (2014).

[60] G. A. Paz-Silva and L. Viola, General Transfer-Function Approach to Noise Filtering in Open-Loop Quantum Control, Phys. Rev. Lett. 113, 250501 (2014).

[61] H. Ball and M. J. Biercuk, Walsh-Synthesized Noise Filters for Quantum Logic, EPJ Quantum Techno. 2, 11 (2015).

[62] A. Soare, H. Ball, D. Hayes, J. Sastrawan, M. C. Jarratt, J. J. McLoughlin, X. Zhen, T. J. Green, and M. J. Biercuk, Experimental Noise Filtering by Quantum Control, Nat. Phys. 10, 825 (2014).

[63] T. J. Green and M. J. Biercuk, Phase-Modulated Decoupling and Error Suppression in Qubit-Oscillator Systems, Phys. Rev. Lett. 114, 120502 (2015).

[64] J. T. Merrill, S. C. Doret, G. Vittorini, J. P. Addison, and K. R. Brown, Transformed Composite Sequences for Improved Qubit Addressing, Phys. Rev. A 90, 040301 (2014).

[65] T. J. Green, J. Sastrawan, H. Uys, and M. J. Biercuk, Arbitrary Quantum Control of Qubits in the Presence of Universal Noise, New J. Phys. 15, 095004 (2013).

[66] T. J. Green, H. Uys, and M. J. Biercuk, High-Order Noise Filtering in Nontrivial Quantum Logic Gates, Phys. Rev. Lett. 109, 020501 (2012).

[67] M. J. Biercuk, A.C. Doherty, and H. Uys, Dynamical Decoupling Sequence Construction as a Filter-Design Problem, J. Phys. B 44, 154002 (2011).

[68] D. Kielpinski, C. Monroe, and D. J. Wineland, Architecture for a Large-Scale Ion-Trap Quantum Computer, Nature (London) 417, 709 (2002).

[69] M. A. Rowe, A. Ben-Kish, B. DeMarco, D. Leibfried, V. Meyer, J. Beall, J. Britton, J. Hughes, W. M. Itano, B. Jelenkovic, C. Langer, T. Rosenband, and D. J. Wineland, Transport of Quantum States and Separation of Ions in a Dual RF Ion Trap, Quantum Inf. Comput. 2, 257 (2002).

[70] M. T. Baig, M. Johanning, A. Wiese, S. Heidbrink, M. Ziolkowski, and C. Wunderlich, A Scalable, Fast, and Multichannel Arbitrary Waveform Generator, Rev. Sci. Instrum. 84, 124701 (2013).

[71] R. Bowler, U. Warring, J. W. Britton, B. C. Sawyer, and J. Amini, Arbitrary Waveform Generator for Quantum Information Processing with Trapped Ions, Rev. Sci. Instrum. 84, 033108 (2013).

[72] R. Bowler, J. Gaebler, Y. Lin, T. R. Tan, D. Hanneke, J. D. Jost, J. P. Home, D. Leibfried, and D. J. Wineland, Coherent Diabatic Ion Transport and Separation in a Multizone Trap Array, Phys. Rev. Lett. 109, 080502 (2012).
[73] T. Ruster, C. Warschburger, H. Kaufmann, C. T. Schmiegelow, A. Walther, M. Hettrich, A. Pfister, V. Kaushal, F. Schmidt-Kaler, and U. G. Poschinger, Experimental Realization of Fast Ion Separation in Segmented Paul Traps, Phys. Rev. A 90, 033410 (2014).

[74] H. Kaufmann, T. Ruster, C. T. Schmiegelow, M. A. Luda, V. Kaushal, J. Schulz, D. von Lindenfels, F. Schmidt-Kaler, and U. G. Poschinger, Fast Ion Swapping for QuantumInformation Processing, Phys. Rev. A 95, 052319 (2017).

[75] A. Walther, F. Ziesel, T. Ruster, S. T. Dawkins, K. Ott, M. Hettrich, K. Singer, F. Schmidt-Kaler, and U. Poschinger, Controlling Fast Transport of Cold Trapped Ions, Phys. Rev. Lett. 109, 080501 (2012).

[76] H. A. Fürst, M. H. Goerz, U. G. Poschinger, M. Murphy, S. Montangero, T. Calarco, F. Schmidt-Kaler, K. Singer, and C. P. Koch, Controlling the Transport of an Ion: Classical and Quantum Mechanical Solutions, New J. Phys. 16, 075007 (2014).

[77] E. Mount, D. Gaultney, G. Vrijsen, M. Adams, S.-Y. Baek, K. Hudek, L. Isabella, S. Crain, A. van Rynbach, P. Maunz, and J. Kim, Scalable Digital Hardware for a Trapped Ion Quantum Computer, Quantum Inf. Process. 15, 5281 (2016).

[78] V. Negnevitsky et al. (unpublished).

[79] D. Kienzler, C. Flhmann, V. Negnevitsky, H.-Y. Lo, M. Marinelli, D. Nadlinger, and J. P. Home, Observation of Quantum Interference between Separated Mechanical Oscillator Wave Packets, Phys. Rev. Lett. 116, 140402 (2016).

[80] F. M. Leupold, M. Malinowski, C. Zhang, V. Negnevitsky, J. Alonso, A. Cabello, and J. P. Home, Sustained StateIndependent Quantum Contextual Correlations from a Single Ion, arXiv:1706.07370.

[81] A. H. Myerson, D. J. Szwer, S. C. Webster, D. T. C. Allcock, M. J. Curtis, G. Imreh, J. A. Sherman, D. N. Stacey, A. M. Steane, and D. M. Lucas, High-Fidelity Readout of Trapped-Ion Qubits, Phys. Rev. Lett. 100, 200502 (2008).

[82] R. Lechner, C. Maier, C. Hempel, P. Jurcevic, B. P. Lanyon, T. Monz, M. Brownnutt, R. Blatt, and C. F. Roos, Electromagnetically-Induced-Transparency Ground-State Cooling of Long Ion Strings, Phys. Rev. A 93, 053401 (2016).

[83] B. M. Terhal, Quantum Error Correction for Quantum Memories, Rev. Mod. Phys. 87, 307 (2015).

[84] C. Horsman, A. G. Fowler, S. Devitt, and R. V. Meter, Surface Code Quantum Computing by Lattice Surgery, New J. Phys. 14, 123011 (2012).

[85] E. Dennis, A. Kitaev, A. Landahl, and J. Preskill, Topological Quantum Memory, J. Math. Phys. (N.Y.) 43, 4452 (2002).

[86] R. Raussendorf and J. Harrington, Fault-Tolerant Quantum Computation with High Threshold in Two Dimensions, Phys. Rev. Lett. 98, 190504 (2007).

[87] S. Bravyi and A. Kitaev, Universal Quantum Computation with Ideal Clifford Gates and Noisy Ancillas, Phys. Rev. A 71, 022316 (2005).

[88] V. Nebendahl, H. Häffner, and C. F. Roos, Optimal Control of Entangling Operations for Trapped-Ion Quantum Computing, Phys. Rev. A 79, 012312 (2009). 
[89] Y. Tomita, M. Gutiérrez, C. Kabytayev, K. R. Brown, M. R. Hutsel, A. P. Morris, K. E. Stevens, and G. Mohler, Comparison of Ancilla Preparation and Measurement Procedures for the Steane [[7,1,3]] Code on a Model Ion-Trap Quantum Computer, Phys. Rev. A 88, 042336 (2013).

[90] A. W. Cross, D. P. DiVincenzo, and B. M. Terhal, A Comparative Code Study for Quantum Fault-Tolerance, Quantum Inf. Comput. 9, 0541 (2009).

[91] K. M. Svore, A. W. Cross, I. L. Chuang, and A. V. Aho, A Flow-Map Model for Analyzing Pseudothresholds in FaultTolerant Quantum Computing, Quantum Inf. Comput. 6, 193 (2006).
[92] R. Chao and B. Reichardt, Quantum Error Correction with Only Two Extra Qubits, arXiv:1705.02329; Fault-Tolerant Quantum Computation with Few Qubits, arXiv:1705.05365.

[93] X. Xu, N. de Beaudrap, J. O'Gorman, and S. C. Benjamin, An Integrity Measure to Benchmark Quantum Error Correcting Memories, arXiv:1707.09951.

[94] J. P. Barnes, C. J. Trout, D. G. Lucarelli, and B. D. Clader, Quantum Error Correction Failure Distributions: Comparison of Coherent and Stochastic Error Models, Phys. Rev. A 95, 062338 (2017). 